\title{
FLUX LIMITED GENERALIZED POROUS MEDIA DIFFUSION EQUATIONS
}

\author{
V. CAselles
}

\begin{abstract}
We study a class of generalized porous media type flux limited diffusion equations and we prove the existence and uniqueness of entropy solutions. We compute the Rankine-Hugoniot condition on the jump set for solutions which are of locally bounded variation in space and time. We give also a geometric characterization of the entropy conditions on the jump set for a restricted class of this type of equations.
\end{abstract}

2010 Mathematics Subject Classification: 35K55, 35K15, 35K65.

Key words: Degenerate parabolic equations, flux limited diffusion, nonlinear semigroup.

\section{Introduction}

We are interested in the quasi-linear parabolic equation

$$
\begin{cases}\frac{\partial u}{\partial t}=\operatorname{div} \mathbf{a}(u, D \Phi(u)) & \text { in } Q_{T}=(0, T) \times \mathbb{R}^{N} \\ u(0, x)=u_{0}(x) & \text { in } x \in \mathbb{R}^{N},\end{cases}
$$

where $0 \leq u_{0} \in L^{1}\left(\mathbb{R}^{N}\right), \mathbf{a}(z, \xi)=\nabla_{\xi} f(z, \xi)$ and $f: \mathbb{R} \times \mathbb{R}^{N} \rightarrow \mathbb{R}^{+}$ is a continuous function, convex in $\xi$, with linear growth as $\|\xi\| \rightarrow \infty$ such that $\mathbf{a}(z, \xi) \in C\left(\mathbb{R} \times \mathbb{R}^{N}\right)$. We assume that $\Phi:[0, \infty) \rightarrow[0, \infty)$ is a strictly increasing function such that $\Phi(0)=0$ with some regularity that will be made precise later on.

When the flux is bounded, this general class of equations is known under the name of flux limited or tempered diffusion equations. One of the first models was proposed by J. R. Wilson in the theory of radiation hydrodynamics [44] and corresponds to the flux $\mathbf{a}(u, D u)=\nu \frac{u D u}{u+\frac{\nu}{c}|D u|}$. In this way, one can enforce the physical restriction that the flux cannot exceed the energy density times the speed of light $c$, that is, the flux cannot violate causality. Another example contained in the general class of models (1.1) is given by the so-called relativistic heat equation $[\mathbf{4 7}]$ 
(see also [25])

$$
u_{t}=\nu \operatorname{div}\left(\frac{u \nabla u}{\sqrt{u^{2}+\frac{\nu^{2}}{c^{2}}|\nabla u|^{2}}}\right) \text {, }
$$

(where $\nu>0$ is a constant representing the kinematic viscosity and $c>0$ is the maximum speed of propagation), one of the relevant examples of the theory. In this case, $\Phi(u)=u$ and the Lagrangian is $f(z, \xi)=$ $\frac{c^{2}}{\nu}|z| \sqrt{z^{2}+\frac{\nu^{2}}{c^{2}}|\xi|^{2}}$.

Rosenau [47] derived (1.2) starting from the observation that the speed of sound is the highest admissible free velocity in a medium. This property is lost in the classical transport theory that predicts the nonphysical divergence of the flux with the gradient, as it happens also with the classical theory of heat conduction (based in Fourier's law) and with the linear diffusion theory (based in Fick's law). To overcome this problem Rosenau [47] proposed to change the classical flux

$$
\mathcal{F}=-\nu \nabla u, \quad \nu>0,
$$

associated with the heat equation (or the Fokker-Plank equation)

$$
u_{t}=\nu \Delta u,
$$

by a flux that saturates as the gradient becomes unbounded. To do that, he associated $u$ and the flux $\mathcal{F}$ through the velocity $\mathbf{v}$ defined by the relation $\mathcal{F}=u \mathbf{v}$. Together with (1.3) this gives

$$
\mathbf{v}=-\nu \frac{\nabla u}{u}
$$

According to (1.5), if $\left|\frac{\nabla u}{u}\right| \uparrow \infty$, so will do $\mathbf{v}$. However, the inertia effects impose a macroscopic upper bound on the allowed free speed, namely, the acoustic speed or light speed $c$. With this aim, Rosenau proposed to modify (1.5) by taking

$$
\nu \frac{\nabla u}{u}=\frac{-\mathbf{v}}{\sqrt{1-\frac{|\mathbf{v}|^{2}}{c^{2}}}} .
$$

The postulate (1.6) forces $\mathbf{v}$ to stay in the subsonic regime (in the case $c$ is the acoustic speed). The sonic limit is approached only if $\left|\frac{\nabla u}{u}\right| \uparrow \infty$. Solving (1.6) for $\mathbf{v}$, we obtain

$$
\mathcal{F}=u \mathbf{v}=\frac{-\nu \nabla u}{\sqrt{1+\left(\frac{\nu|\nabla u|}{c u}\right)^{2}}} .
$$


Using this new flux (1.7) in the conservation energy equation, we obtain (1.2).

The same argument can be applied if, as in Rosenau's proposal, we modify Darcy's law and define $\mathbf{v}$ by

$$
\nu \nabla u^{m}=\frac{-\mathbf{v}}{\sqrt{1-\frac{|\mathbf{v}|^{2}}{c^{2}}}}, \quad m>0 .
$$

In that case the flux is

$$
\mathcal{F}=u \mathbf{v}=\frac{-u \nabla u^{m}}{\sqrt{1+\frac{\nu^{2}}{c^{2}}\left|\nabla u^{m}\right|^{2}}},
$$

and we obtain the flux limited porous media equation $[\mathbf{4 7}]$

$$
u_{t}=\nu \operatorname{div}\left(\frac{u \nabla u^{m}}{\sqrt{1+\frac{\nu^{2}}{c^{2}}\left|\nabla u^{m}\right|^{2}}}\right) .
$$

Many other models of nonlinear degenerate parabolic equations with flux saturation as the gradient becomes unbounded have been proposed by Rosenau and his coworkers $[\mathbf{3 3}],[\mathbf{4 6}],[\mathbf{4 7}]$. In $[\mathbf{3 3}]$ the authors exhibited some models for which initial conditions of compact support, even if smooth, develop a discontinuity in finite time and displayed experiments to show the evolution of the support of its solutions. The same phenomenon of breaking of solutions and apparition of discontinuities was proved by Bertsch and Dal Passo in [17], [38] for equations of type $u_{t}=\left(\varphi(u) \mathbf{b}\left(u_{x}\right)\right)_{x}$, where $\varphi: \mathbb{R} \rightarrow \mathbb{R}^{+}$is smooth and strictly positive, and $\mathbf{b}: \mathbb{R} \rightarrow \mathbb{R}$ is a smooth odd function such that $\mathbf{b}^{\prime}>0$ and $\lim _{s \rightarrow \infty} \mathbf{b}(s)=\mathbf{b}_{\infty}$, which model heat and mass transfer in turbulent fluids [17]. Also in a one-dimensional case and for the Neumann problem, more general flux functions were considered by Blanc in [19], [20]. For such problem, after observing that there are no, in general, classical solutions, the author associated an $m$-accretive operator to the expression $-\left(\mathbf{a}\left(u, u_{x}\right)\right)_{x}$ with Neumann boundary conditions and proved the existence and uniqueness of a semigroup solution. However, the accretive operator generating the semigroup was not characterized in distributional terms. An example of the equations considered in [19], [20], $[\mathbf{4 7}]$ is the so called plasma equation (see [41])

$$
\frac{\partial u}{\partial t}=\left(\frac{u^{5 / 2} u_{x}}{1+u\left|u_{x}\right|}\right)_{x} \quad \text { in } \quad(0, T) \times(0,1),
$$


where the initial condition $u_{0}$ is assumed to be positive. In this case $u$ represents the temperature of electrons and the form of the conductivity $\mathbf{a}\left(u, u_{x}\right)=\frac{u^{5 / 2} u_{x}}{1+u\left|u_{x}\right|}$ has the effect of limiting heat flux.

Up to now, all efforts have been devoted to study the problem (1.1) when $\Phi(r)=r$ (let us point out that these techniques cover the case of sufficiently smooth $\Phi)$. Let us briefly review them. Using the CrandallLiggett's iterative scheme [35] and the notion of entropy solutions, a general existence and uniqueness theory for (1.1) when $\Phi(r)=r$ has been developed in [5], [6] when the initial condition $0 \leq u_{0} \in L^{1}\left(\mathbb{R}^{N}\right) \cap L^{\infty}\left(\mathbb{R}^{N}\right)$ and later extended to initial conditions $u_{0} \in B V\left(\mathbb{R}^{N}\right)$ for $(1.2)$ in $[\mathbf{2 9}]$. The case of the Neumann problem in a bounded domain was previously considered in $[\mathbf{3}],[\mathbf{4}]$ when the initial condition $u_{0} \geq 0$ was bounded and bounded away from 0 or the Lagrangian was coercive. Then equation (1.2) has been the object of special attention. Besides Rosenau's derivation [47], it was also formally derived by Brenier by means of Monge-Kantorovich's mass transport theory in [25], where he named it as the relativistic heat equation. Equation (1.2) has been studied in detail in [8], where the existence of discontinuity fronts propagating at the speed $c$ has been shown. Recently, following the strategy suggested by Brenier [25], McCann and Puel [43] have constructed solutions of the Neumann problem associated with equation (1.2) for bounded initial data assuming that they are also bounded from below. For that, they considered (1.2) as the gradient flow of the Boltzmann entropy for the Wasserstein metric corresponding to the cost function

$$
k(z):= \begin{cases}c^{2}\left(1-\sqrt{1-\frac{|z|^{2}}{c^{2}}}\right) & \text { if }|z| \leq c \\ +\infty & \text { if }|z|>c .\end{cases}
$$

Our main purpose here is to prove existence and uniqueness results for (1.1) to cover the case where $u_{0} \in L^{1}\left(\mathbb{R}^{N}\right) \cap L^{\infty}\left(\mathbb{R}^{N}\right), u_{0} \geq 0$, thus, extending the results in $[\mathbf{5}],[\mathbf{6}]$. We consider here that $\Phi:[0, \infty) \rightarrow$ $[0, \infty)$ is a strictly increasing function such that $\Phi(0)=0, \Phi, \Phi^{-1} \in$ $W^{1, \infty}([a, b])$ for any $0<a<b$.

Our strategy is also based on the use of Crandall-Liggett's iteration scheme [35]. For that, we first consider the elliptic problem

$$
u-\operatorname{div} \mathbf{a}(u, D \Phi(u))=v \quad \text { in } \quad \mathbb{R}^{N},
$$

we define a notion of entropy solution for it, and we prove existence and uniqueness results when the right hand side $0 \leq v \in L^{1}\left(\mathbb{R}^{N}\right) \cap L^{\infty}\left(\mathbb{R}^{N}\right)$. 
We observe that the results for (1.12) can be proved using the condition

$$
|\mathbf{a}(z, \xi)| \leq C z^{\bar{m}} \quad \forall z \in \mathbb{R}^{+}, \quad \bar{m} \geq 1,
$$

(see Subsection 3.1 for the precise set of assumptions on a). The notion of entropy solution permits to prove a uniqueness result based on Kružkov's technique of doubling variables [42] (see [28] for an extension to the second order case) suitably adapted to work with functions whose truncatures are of bounded variation [5], [6]. This last condition is necessary since, in general we are only able to prove that $\min (\max (u, a), b)-a \in B V\left(\mathbb{R}^{N}\right), 0<a<b$. To use Kružkov's technique we need to use test functions of the form $S(\Phi(u)) T(\Phi(u))$ where $S(r)$ is a truncature function of the form $\min (\max (r, a), b)-a, a>0$, used to guarantee that $S(\Phi(u)) T(\Phi(u)) \in B V\left(\mathbb{R}^{N}\right)$, and $T(r)$ is a truncature that will approximate the $\operatorname{sign}_{0}^{+}(r-k), r, k \in \mathbb{R}$. Let us point out that we consider here a more general class of test functions. This has a double purpose: on one hand it permits to clarify the notion of entropy sub and supersolutions, and its connection with the notion of entropy solutions; on the other, it has been useful in the paper [30] in order to interpret the meaning of entropy conditions in the discontinuity fronts of the solutions. The same interpretation holds in our present case.

Let us mention here that there is an extensive literature on renormalized or entropy solutions for elliptic and parabolic problems [11], [12], $[\mathbf{1 3}],[\mathbf{1 7}],[\mathbf{2 1}],[\mathbf{2 2}],[\mathbf{2 3}],[\mathbf{2 7}],[\mathbf{2 8}],[37],[38],[\mathbf{2 4}],[45]$, to mention a few of them. The only ones in this list dealing with flux limited diffusion equations, in one space variable, are $[\mathbf{1 7}],[\mathbf{3 8}]$.

The existence and uniqueness result for (1.12) permits us to associate an accretive operator $B$ in $L^{1}\left(\mathbb{R}^{N}\right)$ whose domain is contained in $\left(L^{1}\left(\mathbb{R}^{N}\right) \cap L^{\infty}\left(\mathbb{R}^{N}\right)\right)^{+}$and whose closure $\mathcal{B}$ is accretive in $L^{1}\left(\mathbb{R}^{N}\right)^{+}$and generates a non-linear contraction semigroup $T(t)$ in that space $[\mathbf{1 6}]$, [35]:

$$
u(t):=T(t) u_{0}=\lim _{n \rightarrow \infty}\left(I+\frac{t}{n} \mathcal{B}\right)^{-n} u_{0}, \quad u_{0} \in L^{1}\left(\mathbb{R}^{N}\right)^{+} .
$$

Then we observe that if $u_{0} \in L^{1}\left(\mathbb{R}^{N}\right) \cap L^{\infty}\left(\mathbb{R}^{N}\right), u_{0} \geq 0$, and the condition (1.13) holds, then $u(t)$ is an entropy solution of (1.1) (a notion that will be defined in Section 5$)$. We also prove that entropy solutions in $\left(L^{1}\left(\mathbb{R}^{N}\right) \cap L^{\infty}\left(\mathbb{R}^{N}\right)\right)^{+}$are unique. As a technical tool to prove these results we need some lower semi-continuity results for energy functionals whose density is a function $g(x, u, D u)$ convex in $D u$ with a linear growth rate as $|D u| \rightarrow \infty$ which were proved in [36] and [39]. Since the proofs 
of these results follow closely the techniques given in $[\mathbf{6}],[\mathbf{2 9}]$ we shall not give them in detail in the parabolic case.

Finally, assuming that the entropy solution $u$ of (1.1) is in $B V_{\text {loc }}\left((0, T) \times \mathbb{R}^{N}\right)$, we compute in Section 6 , Proposition 6.5 , the Rankine-Hugoniot condition on the jump set of $u$. The Rankine-Hugoniot condition gives the speed of the moving discontinuity fronts of $u$. Then, as in [30], we give a characterization of the entropy conditions on the jump set of $u$ as a set of inequalities which have a more geometric interpretation. We are able to find entropy solutions $u \in B V_{\text {loc }}\left((0, T) \times \mathbb{R}^{N}\right)$ if we restrict the model (1.1) and consider the equation

$$
u_{t}=\kappa \operatorname{div}\left(\frac{\Lambda(u) \nabla \Phi(u)}{\sqrt{1+\beta|\nabla \Phi(u)|^{2}}}\right),
$$

where $\beta>0$, and $\Lambda(u), \Phi(u)$ satisfy some additional regularity properties. In this case, $f(z, \xi)=\frac{1}{\beta} \Lambda(z) \sqrt{1+\beta|\xi|^{2}}$. A particular case is given by the choice of $\Lambda(u)=u^{r}, \Phi(u)=u^{m}, r \geq 1, m>0$, and any $u \geq 0$. Together with the Rankine-Hugoniot condition, this permits a full specification of the velocity $v$ of the moving discontinuity fronts of the solution $u$ of (1.14):

$$
v=\frac{\Lambda\left(u^{+}\right)-\Lambda\left(u^{-}\right)}{u^{+}-u^{-}},
$$

where $u^{+}$and $u^{-}$are the upper and lower limits at both sides of the jump set of $u$ (see Section 2 for a precise definition).

Let us explain the plan of the paper. In Section 2 we recall some basic facts about functions of bounded variation, denoted by $B V$, Green's formula, and lower semi-continuity results for energy functionals defined in $B V$. In Section 3 we define the notion of entropy solution (Subsection 3.3) for the elliptic problem (1.12) and prove an existence and uniqueness result for it when the right hand side $v$ of (1.12) is a nonnegative function in $L^{1}\left(\mathbb{R}^{N}\right) \cap L^{\infty}\left(\mathbb{R}^{N}\right)$ (Subsection 3.4). For that, we first discuss in Subsection 3.1 the set of assumptions on $f(z, \xi)$ and $\mathbf{a}(z, \xi)$, and define in Subsection 3.2 a functional calculus adapted to the use of test functions of the form $S(\Phi(u)) T(\Phi(u))$, where $S, T$ are suitable nonlinear Lipschitz functions. In Section 4 we define an accretive operator associated to $-\operatorname{div} \mathbf{a}(u, D u)$ whose closure generates a contraction semigroup in $L^{1}\left(\mathbb{R}^{N}\right)^{+}$. In Section 5 we define the notion of entropy solutions for the evolution problem (1.1) and we state the existence and uniqueness of entropy solutions for any initial datum $u_{0} \in\left(L^{1}\left(\mathbb{R}^{N}\right) \cap L^{\infty}\left(\mathbb{R}^{N}\right)\right)^{+}$. In the parabolic case, the proofs will not be given in detail and can be reconstructed using the methods in $[\mathbf{6}],[\mathbf{2 9}]$ together with the necessary 
adaptations that are used in Subsection 3.4 for the stationary case. Finally, in Section 6 we apply our previous results to the class of equations (1.14) under suitable assumptions on $\Lambda, \Phi$. In particular, in Subsection 6.2 we compute the Rankine-Hugoniot condition on the jump set of $u$ for solutions $u$ which are in $B V_{\text {loc }}\left((0, T) \times \mathbb{R}^{N}\right)$. Sufficient conditions that imply that $u \in B V_{\text {loc }}\left((0, T) \times \mathbb{R}^{N}\right)$ are given in Subsection 6.1. In Subsection 6.2 we give also a characterization of the entropy conditions on the jump set of $u$ as a set of inequalities which have a more geometric interpretation.

\section{Preliminaries}

2.1. Functions of bounded variation. Let us start with some notation. We denote by $\mathcal{L}^{N}$ and $\mathcal{H}^{N-1}$ the $N$-dimensional Lebesgue measure and the $(N-1)$-dimensional Hausdorff measure in $\mathbb{R}^{N}$, respectively. Given an open set $\Omega$ in $\mathbb{R}^{N}$ we shall denote by $\mathcal{D}(\Omega)$ the space of infinitely differentiable functions with compact support in $\Omega$. We denote by $C_{c}(\Omega)$, resp. $C_{c}^{k}(\Omega)(k \in \mathbb{N} \cup\{\infty\})$, the set of continuous, resp. $k$-times continuously differentiable, functions with compact support in $\Omega$.

Due to the linear growth condition on the Lagrangian, the natural energy space to study (1.1) is the space of functions of bounded variation. We briefly recall some facts about functions of bounded variation (for further information about them we refer to $[\mathbf{1}]$ ).

Let $\Omega$ be an open subset of $\mathbb{R}^{N}$. A function $u \in L^{1}(\Omega)$ whose gradient $D u$ in the sense of distributions is a (vector valued) Radon measure with finite total variation in $\Omega$ is called a function of bounded variation. The class of such functions will be denoted by $B V(\Omega)$. The total variation of $D u$ in $\Omega$ turns out to be

$$
\sup \left\{\int_{\Omega} u \operatorname{div} \sigma d x: \sigma \in C_{c}^{\infty}\left(\Omega ; \mathbb{R}^{N}\right),|\sigma(x)| \leq 1 \forall x \in \Omega\right\},
$$

(where for a vector $v=\left(v_{1}, \ldots, v_{N}\right) \in \mathbb{R}^{N}$ we set $|v|^{2}:=\sum_{i=1}^{N} v_{i}^{2}$ ) and will be denoted by $|D u|(\Omega)$, or by $\int_{\Omega}|D u|$. The map $u \rightarrow|D u|(\Omega)$ is $L_{\text {loc }}^{1}(\Omega)$-lower semicontinuous. $B V(\Omega)$ is a Banach space when endowed with the norm $\|u\|:=\int_{\Omega}|u| d x+|D u|(\Omega)$. If $\Omega=\mathbb{R}^{N}$, we consider $B V\left(\mathbb{R}^{N}\right)$ endowed with norm $\|u\|_{B V\left(\mathbb{R}^{N}\right)}=|D u|\left(\mathbb{R}^{N}\right)$. We recall that $B V(\Omega) \subset L^{p}(\Omega)$ for any $1 \leq p \leq \frac{N}{N-1}$, the embedding being compact if $\Omega$ is bounded with Lipschitz boundary and $p<\frac{N}{N-1}$.

A measurable set $E \subseteq \Omega$ is said to be of finite perimeter in $\Omega$ if (2.1) is finite when $u$ is substituted with the characteristic function $\chi_{E}$ 
of $E$. The perimeter of $E$ in $\Omega$ is defined as $P(E, \Omega):=\left|D \chi_{E}\right|(\Omega)$. If $\Omega=\mathbb{R}^{N}$, we denote $P(E):=P\left(E, \mathbb{R}^{N}\right)$.

For $u \in B V(\Omega)$, the gradient $D u$ is a Radon measure that decomposes into its absolutely continuous and singular parts $D u=D^{a} u+D^{s} u$. Then $D^{a} u=\nabla u \mathcal{L}^{N}$ where $\nabla u$ is the Radon-Nikodym derivative of the measure $D u$ with respect to the Lebesgue measure $\mathcal{L}^{N}$. Let us denote by $D^{s} u=\overrightarrow{D^{s} u}\left|D^{s} u\right|$ the polar decomposition of $D^{s} u$, where $\left|D^{s} u\right|$ is the total variation measure of $D^{s} u$. We also split $D^{s} u$ in two parts: the jump part $D^{j} u$ and the Cantor part $D^{c} u$.

We say that $u$ is approximately continuous at the point $x \in \Omega$ if there exists $\tilde{u}(x) \in \mathbb{R}$ such that

$$
\lim _{r \downarrow 0} \frac{1}{\mathcal{L}^{N}\left(B_{r}(x)\right)} \int_{B_{r}(x)}|u(y)-\tilde{u}(x)| d y=0,
$$

the value $\tilde{u}(x)$ is called the approximate limit of $u$ at $x$. We denote by $S_{u}$ the set of all $x \in \Omega$ such that $u$ is not approximately continuous at $x$. We say that $x \in \Omega$ is an approximate jump point of $u$ if there exist $u^{+}(x) \neq u^{-}(x) \in \mathbb{R}$ and $\nu_{u}(x) \in S^{N-1}$ such that

$$
\begin{aligned}
& \lim _{\rho \downarrow 0} \frac{1}{\mathcal{L}^{N}\left(B_{\rho}^{+}\left(x, \nu_{u}(x)\right)\right)} \int_{B_{\rho}^{+}\left(x, \nu_{u}(x)\right)}\left|u(y)-u^{+}(x)\right| d y=0 \\
& \lim _{\rho \downarrow 0} \frac{1}{\mathcal{L}^{N}\left(B_{\rho}^{-}\left(x, \nu_{u}(x)\right)\right)} \int_{B_{\rho}^{-}\left(x, \nu_{u}(x)\right)}\left|u(y)-u^{-}(x)\right| d y=0,
\end{aligned}
$$

where

$$
B_{\rho}^{+}\left(x, \nu_{u}(x)\right)=\left\{y \in B_{\rho}(x):\left\langle y-x, \nu_{u}(x)\right\rangle>0\right\}
$$

and

$$
B_{\rho}^{-}\left(x, \nu_{u}(x)\right)=\left\{y \in B_{\rho}(x):\left\langle y-x, \nu_{u}(x)\right\rangle<0\right\} .
$$

We denote by $J_{u}$ the set of approximate jump points of $u$. $J_{u}$ is a Borel subset of $S_{u}$ and $\mathcal{H}^{N-1}\left(S_{u} \backslash J_{u}\right)=0$. We have

$$
D^{j} u=D^{s} u\left\llcorner J_{u} \quad \text { and } \quad D^{c} u=D^{s} u\left\llcorner\left(\Omega \backslash S_{u}\right) .\right.\right.
$$

It is well known (see for instance [1]) that

$$
D^{j} u=\left(u^{+}-u^{-}\right) \nu_{u} \mathcal{H}^{N-1}\left\llcorner J_{u} .\right.
$$

Moreover, if $x \in J_{u}$, then $\nu_{u}(x)=\frac{D u}{|D u|}(x)$, $\frac{D u}{|D u|}$ being the RadonNikodym derivative of $D u$ with respect to its total variation $|D u|$. 
2.2. Lower semicontinuity of functionals defined on $B \boldsymbol{V}$. Let $\Omega$ be an open subset of $\mathbb{R}^{N}$. Given a Borel function $g: \Omega \times \mathbb{R} \times \mathbb{R}^{N} \rightarrow[0, \infty[$, we consider the energy functional

$$
G(w):=\int_{\Omega} g(x, w(x), \nabla w(x)) d x
$$

defined in the Sobolev space $W^{1,1}(\Omega)$. In order to get an integral representation of the relaxed energy associated with $G$, i.e.,

$$
\mathcal{G}(w):=\inf _{\left\{w_{n}\right\}}\left\{\liminf _{n \rightarrow \infty} G\left(w_{n}\right): w_{n} \in W^{1,1}(\Omega), w_{n} \rightarrow w \in L^{1}(\Omega)\right\},
$$

Dal Maso introduced in [36] the following functional for $w \in B V(\Omega)$ :

$$
\begin{aligned}
\mathcal{R}_{g}(w):= & \int_{\Omega} g(x, w(x), \nabla w(x)) d x \\
& +\int_{\Omega} g^{0}\left(x, \tilde{w}(x), \frac{D w}{|D w|}(x)\right)\left|D^{c} w\right| \\
& +\int_{J_{w}}\left(\int_{w_{-}(x)}^{w_{+}(x)} g^{0}\left(x, s, \nu_{w}(x)\right) d s\right) d \mathcal{H}^{N-1}(x),
\end{aligned}
$$

where the recession function $g^{0}$ of $g$ is defined as

$$
g^{0}(x, z, \xi)=\lim _{t \rightarrow 0^{+}} t g\left(x, z, \frac{\xi}{t}\right) .
$$

It is clear that the function $g^{0}(x, z, \xi)$ is positively homogeneous of degree one in $\xi$, i.e.

$$
g^{0}(x, z, s \xi)=s g^{0}(x, z, \xi) \quad \text { for all } z, \xi \text { and } s>0 .
$$

In case that $\Omega$ is a bounded set, and under standard continuity and coercivity assumptions, Dal Maso proved in $[\mathbf{3 6}]$ that $\mathcal{G}(w)=\mathcal{R}_{g}(w)$ for all $w \in B V(\Omega)$. More recently, De Cicco, Fusco, and Verde in [39], have obtained a very general result about the $L^{1}$-lower semi-continuity of $\mathcal{R}_{g}$ in $B V$ :

Theorem 2.1. Let $\Omega$ be an open subset of $\mathbb{R}^{N}$ and $g: \Omega \times \mathbb{R} \times \mathbb{R}^{N} \rightarrow[0, \infty[$ a locally bounded Carathéodory function such that, for every $(z, \xi) \in$ $\mathbb{R} \times \mathbb{R}^{N}$, the function $g(\cdot, z, \xi)$ is of class $C^{1}$. Let us assume that

(i) $g(x, z, \cdot)$ is convex in $\mathbb{R}^{N}$ for every $(x, z) \in \Omega \times \mathbb{R}$,

(ii) $g(x, \cdot, \xi)$ is continuous in $\mathbb{R}$ for every $(x, \xi) \in \Omega \times \mathbb{R}^{N}$.

Then, the functional $\mathcal{R}_{g}(u)$ is lower semi-continuous respect to the $L^{1}(\Omega)$-convergence. 
Let $f: \mathbb{R} \times \mathbb{R}^{N} \rightarrow[0, \infty[$ a continuous function, such that there exists $f^{0}$ and $\left|f^{0}(z, \xi)\right| \leq C\|\xi\|$ for any $z \in \mathbb{R}, \xi \in \mathbb{R}^{N}$, and some $C>0$. Given a function $w \in B V\left(\mathbb{R}^{N}\right)$, we define the Radon measure $f(w, D w)$ in $\mathbb{R}^{N}$ as

$$
\langle f(w, D w), \phi\rangle:=\mathcal{R}_{\phi f}(w), \quad \phi \in C_{c}\left(\mathbb{R}^{N}\right) .
$$

Let us observe that if $f^{0}(z, \xi)=\varphi(z) \psi^{0}(\xi)$, with $\varphi$ Lipschitz continuous and $\psi^{0}$ a convex function homogeneous of degree 1 , by applying the chain rule for BV-functions (see $[\mathbf{1}]$ ), we have

$$
\mathcal{R}_{\phi f}(w)=\int_{\mathbb{R}^{N}} \phi(x) f(w, \nabla w) d x+\int_{\mathbb{R}^{N}} \phi(x) \psi^{0}\left(\frac{D w}{|D w|}\right)\left|D^{s} J_{\varphi}(w)\right|,
$$

where $J_{\varphi}(r)=\int_{0}^{r} \varphi(s) d s$. Then, under these conditions, we have

$$
f(w, D w)^{s}=\psi^{0}\left(\frac{D w}{|D w|}\right)\left|D^{s} J_{\varphi}(w)\right| .
$$

2.3. Coupling between a vector field and the gradient of a function. We shall need several results from $[\mathbf{1 0}]$ in order to give a sense to the integrals of bounded vector fields with divergence in $L^{p}$ integrated with respect to the gradient of a $B V$ function. Assume that $\Omega=\mathbb{R}^{N}$ or $\Omega$ is an open bounded set of $\mathbb{R}^{N}$ with Lipschitz continuous boundary. We denote by $\nu^{\Omega}(x)$ the outer unit normal to $\partial \Omega$ at $x \in \partial \Omega$.

Let $p \geq 1$. Let us denote

$$
X_{p}(\Omega)=\left\{\mathbf{z} \in L^{\infty}\left(\Omega, \mathbb{R}^{N}\right): \operatorname{div}(\mathbf{z}) \in L^{p}(\Omega)\right\} .
$$

If $\mathbf{z} \in X_{p}(\Omega)$ and $w \in L^{p^{\prime}}(\Omega)$, where $\frac{1}{p}+\frac{1}{p^{\prime}}=1$, we define the functional $(\mathbf{z} \cdot D w): C_{c}^{\infty}(\Omega) \rightarrow \mathbb{R}$ by the formula

$$
\langle(\mathbf{z} \cdot D w), \varphi\rangle:=-\int_{\Omega} w \varphi \operatorname{div}(\mathbf{z}) d x-\int_{\Omega} w \mathbf{z} \cdot \nabla \varphi d x, \quad \varphi \in C_{c}^{\infty}(\Omega) .
$$

If $\mathbf{z} \in X_{p}(\Omega)$ and $w \in B V(\Omega) \cap L^{p^{\prime}}(\Omega)$, then $(\mathbf{z} \cdot D w)$ is a Radon measure in $\Omega[\mathbf{1 0}]$, and

$$
\int_{\Omega}(\mathbf{z} \cdot D w)=\int_{\Omega} \mathbf{z} \cdot \nabla w d x, \quad \forall w \in W^{1,1}(\Omega) \cap L^{\infty}(\Omega) .
$$

Moreover, $(\mathbf{z} \cdot D w)$ is absolutely continuous with respect to $|D w|[\mathbf{1 0}]$.

In the case where the distribution $(\mathbf{z} \cdot D w)$ is a Radon measure we denote by $(\mathbf{z} \cdot D w)^{a c},(\mathbf{z} \cdot D w)^{s}$ its absolutely continuous and singular parts with respect to $\mathcal{L}^{N}$. 
The weak trace on $\partial \Omega$ of the normal component of $\mathbf{z} \in X_{p}(\Omega)$ is defined in [10]. More precisely, it is proved that there exists a linear operator $\gamma: X_{p}(\Omega) \rightarrow L^{\infty}(\partial \Omega)$ such that

$$
\begin{gathered}
\|\gamma(\mathbf{z})\|_{\infty} \leq\|\mathbf{z}\|_{\infty} \\
\gamma(\mathbf{z})(x)=\mathbf{z}(x) \cdot \nu^{\Omega}(x) \text { for all } x \in \partial \Omega \text { if } \mathbf{z} \in C^{1}\left(\bar{\Omega}, \mathbb{R}^{N}\right) .
\end{gathered}
$$

We shall denote $\gamma(\mathbf{z})(x)$ by $\left[\mathbf{z} \cdot \nu^{\Omega}\right](x)$. Moreover, the following Green's formula, relating the function $\left[\mathbf{z} \cdot \nu^{\Omega}\right]$ and the measure $(\mathbf{z} \cdot D w)$ for $\mathbf{z} \in$ $X_{p}(\Omega)$ and $w \in B V(\Omega) \cap L^{p^{\prime}}(\Omega)$, holds (see $[\mathbf{1 0}]$ )

$$
\int_{\Omega} w \operatorname{div}(\mathbf{z}) d x+\int_{\Omega}(\mathbf{z} \cdot D w)=\int_{\partial \Omega}\left[\mathbf{z} \cdot \nu^{\Omega}\right] w d \mathcal{H}^{N-1} .
$$

If $\Omega=\mathbb{R}^{N}$, Green's formula is

$$
\int_{\mathbb{R}^{N}} w \operatorname{div}(\mathbf{z}) d x+\int_{\mathbb{R}^{N}}(\mathbf{z} \cdot D w)=0 .
$$

The same results hold if we assume that $\mathbf{z} \in L^{\infty}\left(\Omega, \mathbb{R}^{N}\right)$ and $\operatorname{div}(\mathbf{z})$ is a Radon measure in $\Omega$, and $w \in B V(\Omega) \cap L^{\infty}(\Omega)[\mathbf{1 0}],[\mathbf{3 0}],[\mathbf{3 2}]$.

2.4. Some function spaces of measurable functions. Let us recall some results of $[\mathbf{1 5}]$ that we need. Let $M\left(\mathbb{R}^{N}\right)$ the set of the Lebesgue measurable mappings from $\mathbb{R}^{N}$ into $\mathbb{R}$. Recall that $L^{1}\left(\mathbb{R}^{N}\right)+L^{\infty}\left(\mathbb{R}^{N}\right)$ with the norm

$\|u\|_{1+\infty}:=\inf \left\{\left\|u_{1}\right\|_{1}+\left\|u_{2}\right\|_{\infty}: u=u_{1}+u_{2},, u_{1} \in L^{1}\left(\mathbb{R}^{N}\right), u_{2} \in L^{\infty}\left(\mathbb{R}^{N}\right)\right\}$

is a Banach space. If we denote

$$
L_{0}\left(\mathbb{R}^{N}\right):=\left\{u \in M\left(\mathbb{R}^{N}\right): \int_{\mathbb{R}^{N}}(|u|-k)^{+}<\infty \quad \forall k>0\right\},
$$

then we have

$$
L_{0}\left(\mathbb{R}^{N}\right)=\overline{L^{1}\left(\mathbb{R}^{N}\right) \cap L^{\infty}\left(\mathbb{R}^{N}\right)} \|_{1+\infty} .
$$

The dual space of $L_{0}\left(\mathbb{R}^{N}\right)$ is isometrically isomorphic to $L^{1}\left(\mathbb{R}^{N}\right) \cap$ $L^{\infty}\left(\mathbb{R}^{N}\right)$, when in $L^{1}\left(\mathbb{R}^{N}\right) \cap L^{\infty}\left(\mathbb{R}^{N}\right)$ is given the norm $\|u\|_{1 \cap \infty}:=$ $\max \left\{\|u\|_{1},\|u\|_{\infty}\right\}$.

Given $u, \bar{u} \in M\left(\mathbb{R}^{N}\right)$, we shall write

$$
u \ll \bar{u} \quad \text { if and only if } \int_{\mathbb{R}^{N}} j(u) d x \leq \int_{\mathbb{R}^{N}} j(\bar{u}) d x
$$

for all

$$
j \in J_{0}:=\{j: \mathbb{R} \rightarrow[0,+\infty] \text {, convex, l.s.c, } j(0)=0\} \text {. }
$$


Let

$$
\mathcal{P}_{0}:=\left\{p \in C^{\infty}(\mathbb{R}): 0 \leq p^{\prime} \leq 1, \operatorname{supp}\left(p^{\prime}\right) \text { compact, } 0 \notin \operatorname{supp}(p)\right\} .
$$

In the next proposition we recall some of the results given in [15].

Proposition 2.2. Let $u \in L_{0}\left(\mathbb{R}^{N}\right)$. Then,

(i) If $v \in L^{1}\left(\mathbb{R}^{N}\right)+L^{\infty}\left(\mathbb{R}^{N}\right)$,

$$
u \ll u+\lambda v \forall \lambda>0 \quad \Longleftrightarrow \quad \int_{\mathbb{R}^{N}} p(u) v \geq 0 \forall p \in \mathcal{P}_{0} .
$$

In particular,

$$
\int_{\mathbb{R}^{N}} u p(u) \leq \int_{\mathbb{R}^{N}} v p(u) \forall p \in \mathcal{P}_{0} \quad \Longrightarrow \quad u \ll v .
$$

(ii) The set $\left\{\bar{u} \in M\left(\mathbb{R}^{N}\right): \bar{u} \ll u\right\}$ is a weakly sequentially compact subset of $L_{0}\left(\mathbb{R}^{N}\right)$.

(iii) If $u \in M\left(\mathbb{R}^{N}\right)$ and $\bar{u} \in L^{p}\left(\mathbb{R}^{N}\right), 1 \leq p \leq \infty$, then

$$
u \ll \bar{u} \quad \Longrightarrow \quad u \in L^{p}\left(\mathbb{R}^{N}\right) \quad \text { and } \quad\|u\|_{p} \leq\|\bar{u}\|_{p} .
$$

Moreover, if $1 \leq p<\infty, u_{n}$ is a sequence satisfying $u_{n} \ll u \in$ $L^{p}\left(\mathbb{R}^{N}\right)$ for all $n \in \mathbb{N}$, and $u_{n} \rightarrow u$ weakly in $L_{0}\left(\mathbb{R}^{N}\right)$, then $u_{n} \rightarrow u$ strongly in $L^{p}\left(\mathbb{R}^{N}\right)$.

\section{The elliptic problem}

The purpose of this section is to prove an existence and uniqueness result for the elliptic problem

$$
u-\operatorname{div} \mathbf{a}(u, D \Phi(u))=v \quad \text { in } \quad \mathbb{R}^{N},
$$

where $v \in\left(L^{1}\left(\mathbb{R}^{N}\right) \cap L^{\infty}\left(\mathbb{R}^{N}\right)\right)^{+}$under some assumptions on a and $\Phi$ that we describe below.

3.1. Assumptions on $\boldsymbol{\Phi}$ and the Lagrangian $\boldsymbol{f}$. We assume that $\Phi:[0, \infty) \rightarrow[0, \infty)$ is a continuous strictly increasing function such that $\Phi(0)=0$ and $\Phi, \Phi^{-1} \in W^{1, \infty}([a, b])$ for any $0<a<b$.

Here we assume that the Lagrangian $f:[0, \infty) \times \mathbb{R}^{N} \rightarrow \mathbb{R}^{+}$satisfies the following assumptions, which we shall refer collectively as $(\mathrm{H})$ :

$\left(\mathrm{H}_{1}\right) f$ is continuous on $[0, \infty) \times \mathbb{R}^{N}$ and is a convex differentiable function of $\xi$ such that $\nabla_{\xi} f(z, \xi) \in C\left([0, \infty) \times \mathbb{R}^{N}\right)$. Further we require $f$ to satisfy the linear growth condition

$$
f(z, \xi) \leq M_{0}(z)(\|\xi\|+1)
$$


for any $(z, \xi) \in[0, \infty) \times \mathbb{R}^{N}$, and the coercivity condition

$$
f(z, \xi) \geq C_{0}(z)\|\xi\|-D_{0}(z)
$$

for any $(z, \xi) \in(0, \infty) \times \mathbb{R}^{N}$ and some positive and continuous functions $C_{0}, M_{0} \in C([0, \infty)), D_{0}(z) \in C(0, \infty)$, such that $C_{0}(z)>0$ for any $z \neq 0$. Moreover, we assume that $f^{0}$ exists. Assume that

$$
C_{0}(z) \geq c_{0}|z|^{\bar{m}} \quad \text { for some } c_{0}>0, \bar{m} \geq 1, z \in[0, \infty) \text {. }
$$

We consider the function $\mathbf{a}(z, \xi)=\nabla_{\xi} f(z, \xi)$ associated to the Lagrangian $f$. By the convexity of $f$

$$
\mathbf{a}(z, \xi) \cdot(\eta-\xi) \leq f(z, \eta)-f(z, \xi) \quad \forall z \in[0, \infty), \forall \eta, \xi \in \mathbb{R}^{N},
$$

and the following monotonicity condition is satisfied

$$
(\mathbf{a}(z, \eta)-\mathbf{a}(z, \xi)) \cdot(\eta-\xi) \geq 0 .
$$

$\left(\mathrm{H}_{2}\right)$ We assume that there is a constant $M>0$ such that

$$
\mathbf{a}(z, \xi)=z^{\bar{m}} \mathbf{b}(z, \xi) \quad \text { with } \quad|\mathbf{b}(z, \xi)| \leq M \forall(z, \xi) \in[0, \infty) \times \mathbb{R}^{N} .
$$

Since $\bar{m} \geq 1$ condition $\left(\mathrm{H}_{2}\right)$ will be used to prove that the flux is integrable when the initial condition is in $\left(L^{1}\left(\mathbb{R}^{N}\right) \cap L^{\infty}\left(\mathbb{R}^{N}\right)\right)^{+}$.

We consider the function $h:[0, \infty) \times \mathbb{R}^{N} \rightarrow \mathbb{R}$ defined by

$$
h(z, \xi):=\mathbf{a}(z, \xi) \cdot \xi .
$$

From (3.5), (3.2) and (3.7), it follows that

$$
C_{0}(z)\|\xi\|-D_{1}(z) \leq h(z, \xi) \leq M|z|^{\bar{m}}\|\xi\|
$$

for any $(z, \xi) \in[0, \infty) \times \mathbb{R}^{N}$, where $D_{1}(z)=D_{0}(z)+f(z, 0)$. The upper inequality holds for $z=0$ and any $\xi$.

$\left(\mathrm{H}_{3}\right)$ We assume that $\frac{\partial \mathbf{a}}{\partial \xi_{i}}(z, \xi) \in C\left([0, \infty) \times \mathbb{R}^{N}\right)$ for any $i=1, \ldots, N$.

An example where this assumption is not necessary was given in [5], [6] for the case where $f(z, \xi)=\varphi(z) \psi(\xi)$ for suitable functions $\varphi$ and $\psi$.

$\left(\mathrm{H}_{4}\right)$ We assume that $h(z, \xi) \geq 0, h(z, \xi)=h(z,-\xi)$ for all $(z, \xi) \in$ $[0, \infty) \times \mathbb{R}^{N}$ and $h^{0}$ exists.

Observe that if we assume that $\mathbf{a}(z, 0)=0$, then (3.6) implies that $h(z, \xi) \geq 0$ for any $z \in[0, \infty)$ and any $\xi \in \mathbb{R}^{N}$.

Observe that we have

$$
c_{0}|z|^{\bar{m}}\|\xi\| \leq h^{0}(z, \xi) \leq M|z|^{\bar{m}}\|\xi\| \quad \text { for any }(z, \xi) \in[0, \infty) \times \mathbb{R}^{N} .
$$


$\left(\mathrm{H}_{5}\right) f^{0}(z, \xi)=h^{0}(z, \xi)$, for all $(z, \xi) \in[0, \infty) \times \mathbb{R}^{N}$.

$\left(\mathrm{H}_{6}\right) \mathbf{a}(z, \xi) \cdot \eta \leq h^{0}(z, \eta)$ for all $\xi, \eta \in \mathbb{R}^{N}$, and all $z \in[0, \infty)$.

$\left(\mathrm{H}_{7}\right)$ We assume that $h^{0}(z, \xi)$ can be written in the form $h^{0}(z, \xi)=$ $\varphi(z) \psi^{0}(\xi)$ where $\varphi$ is a Lipschitz continuous function such that $c_{0}|z|^{\bar{m}} \leq$ $\varphi(z) \leq C|z|^{\bar{m}}$, for some $C>0$ and for any $z \in[0, \infty)$, and $\psi^{0}$ is a convex function homogeneous of degree 1 .

$\left(\mathrm{H}_{8}\right)$ There is a constant $C>0$ such that

$$
\mid \mathbf{a}(z, \xi)-\mathbf{a}(\hat{z}, \xi))|\leq C| z^{\bar{m}}-\hat{z}^{\bar{m}} \mid
$$

for any $(z, \xi),(\hat{z}, \xi) \in[0, \infty) \times \mathbb{R}^{N}$.

Observe that, by the monotonicity condition (3.6) and using (3.9), it follows that

$$
(\mathbf{a}(z, \xi)-\mathbf{a}(\hat{z}, \hat{\xi})) \cdot(\xi-\hat{\xi}) \geq-C\left|z^{\bar{m}}-\hat{z}^{\bar{m}}\right|\|\xi-\hat{\xi}\|
$$

for any $(z, \xi),(\hat{z}, \hat{\xi}) \in[0, \infty) \times \mathbb{R}^{N}$.

Remark 3.1. There are physical models for plasma fusion by inertial confinement in which the temperature evolution of the electrons satisfies an equation of type (1.1), where $\Phi(z)=z, \mathbf{a}(z, \xi)=\frac{z^{5 / 2} \xi}{1+z|\xi|}$ which corresponds to $f(z, \xi)=z^{3 / 2}|\xi|-z^{1 / 2} \ln (1+z|\xi|$ ) [41], (see also [19] for a mathematical study in the one-dimensional case). In this case $\bar{m}=\frac{3}{2}$ and $h^{0}(z, \xi)=z^{3 / 2}|\xi|$. As observed in [5], [6], the assumptions $\left(\mathrm{H}_{1}\right)-\left(\mathrm{H}_{8}\right)$ hold.

Remark 3.2. The function $f(z, \xi)=\frac{c^{2}}{\nu} z \sqrt{z^{2}+\frac{\nu^{2}}{c^{2}}|\xi|^{2}}$ satisfies the assumptions $\left(\mathrm{H}_{1}\right)-\left(\mathrm{H}_{8}\right)$, with $a(z, \xi)=\nu \frac{z \xi}{\sqrt{z^{2}+\frac{\nu^{2}}{c^{2}}|\xi|^{2}}}$ and $\bar{m}=1$. If we take $\Phi(z)=z$, this particular case is related to the so-called relativistic heat equation (1.2) [47], [25], $c$ being a bound of the propagation speed and $\nu$ being a constant representing a kinematic viscosity. Taking $\Phi(z)=z^{m}, z \geq 0$, we get the PDE (1.9). Another example is given by the Lagrangian $f(z, \xi):=c z\left(|\xi|-\frac{c z}{\nu} \log \left(1+\frac{\nu}{c z}|\xi|\right)\right)$ which appears in the theory of radiation hydrodynamics [44]. In this case $\Phi(z)=z$, $a(z, \xi)=\nu \frac{z \xi}{z+\frac{\nu}{c}|\xi|}$ and $\bar{m}=1$.

Remark 3.3. The case of the flux-limited porous media equation (1.9) can also be covered by taking $\Phi(z)=z$. More generally, consider the function $f(z, \xi)=\alpha z^{r-2 p} \sqrt{1+\beta z^{2 p}|\xi|^{2}}-\alpha z^{r-2 p}$, so that $\mathbf{a}(z, \xi)=\frac{\alpha \beta z^{r} \xi}{\sqrt{1+\beta z^{2 p}|\xi|^{2}}}$. 
If $r \geq p+1$, then $\left(\mathrm{H}_{1}\right)-\left(\mathrm{H}_{2}\right)$ hold, in particular (3.2), (3.3) and (3.4). We notice that $r \geq p+1$ is needed for the right inequality in (3.2), for (3.4) and for (3.7). Clearly, $\left(\mathrm{H}_{3}\right)-\left(\mathrm{H}_{7}\right)$ hold. Also $\left(\mathrm{H}_{8}\right)$ holds since $\left|\frac{\partial \mathbf{a}}{\partial z}\right| \leq r z^{r-p-1}+z^{r}$, which is bounded when $r \geq p+1$. The porous media type equation $u_{t}=\operatorname{div}\left(\frac{u^{\kappa} \nabla u^{m}}{1+\left|\nabla u^{m}\right|^{2}}\right)$, corresponds to $\alpha=\frac{1}{m}, \beta=m^{2}$, $r=\kappa+m-1, p=m-1$. Then $r \geq p+1$ is equivalent to $\kappa \geq 1$ When compared to the approach in $[\mathbf{5}],[\mathbf{6}]$, we point out that by examining the proof the term $D_{0}(z)$ in the inequality in (3.3) is only used in the context of the inequality $(3.8)$ and we only need that $D_{1}(z)$ is bounded when $z \in[a, b], 0<a<b$, and that $f(z, 0)$ is bounded for bounded $z$. But this is implied by $\left(\mathrm{H}_{1}\right)$. Thus, the exponents are only restricted by the condition $r \geq p+1$. That is, we only need $\kappa \geq 1$ and $m>0$.

Remark 3.4. More generally, we consider the function $f(z, \xi)=\frac{\psi(z)}{\chi(z)^{2}} \sqrt{1+\chi(z)^{2}|\xi|^{2}}-\frac{\psi(z)}{\chi(z)^{2}}$, where $\psi \in C([0, \infty)), \chi \in C(0, \infty)$. We have $\mathbf{a}(z, \xi)=\frac{\psi(z) \xi}{\sqrt{1+\chi(z)^{2}|\xi|^{2}}}$. To be more precise, let us consider the equation $u_{t}=\operatorname{div}\left(\frac{u^{\kappa} \nabla \Phi(u)}{1+|\nabla \Phi(u)|^{2}}\right)$. In this case, $\psi=z^{\kappa} \Phi^{\prime}$ and $\chi=\Phi^{\prime}$. The right hand side of the inequality (3.2) holds with $M_{0}(z)=\frac{\psi(z)}{\chi(z)}=z^{\kappa}$. We also have $C_{0}(z)=\frac{\psi(z)}{\chi(z)}=z^{\kappa}$. Thus, we need that $\kappa \geq 1$. Then the conditions required in $\left(\mathrm{H}_{1}\right)-\left(\mathrm{H}_{2}\right)$ hold. Conditions $\left(\mathrm{H}_{3}\right)-\left(\mathrm{H}_{7}\right)$ also hold. Since we have $\left|\frac{\partial \mathbf{a}}{\partial z}\right| \leq \psi+\frac{\psi^{\prime}}{\chi}$, to prove $\left(\mathrm{H}_{8}\right)$ we need to ensure that $\left|\frac{\partial \mathbf{a}}{\partial z}\right|$ is bounded. For that we need to assume that $\kappa \geq 1$, and $z^{\kappa} \Phi^{\prime}$ and $z^{k} \frac{\Phi^{\prime \prime}}{\Phi^{\prime}}$ are bounded for bounded $z$. This is satisfied in the case of porous media, but not in general. Thus, to cover the PDE (1.14) the present formulation is more general than the formulation in $[\mathbf{5}],[\mathbf{6}]$.

\subsection{A functional calculus.}

3.2.1. Preparation of the functional calculus. We need to consider the following truncature functions. For $a<b$, let $T_{a, b}(r):=$ $\max (\min (b, r), a)$. As usual, we denote $T_{k}=T_{-k, k}$. We also consider the truncature functions of the form $T_{a, b}^{l}(r):=T_{a, b}(r)-l(l \in \mathbb{R})$. We denote

$$
\mathcal{T}_{r}:=\left\{T_{a, b}: 0<a<b\right\}, \quad \mathcal{T}^{+}:=\left\{T_{a, b}^{l}: 0<a<b, l \in \mathbb{R}, T_{a, b}^{l} \geq 0\right\} .
$$

We denote by $\mathcal{P}$ the set

$\mathcal{P}:=\left\{p:\left[0,+\infty\left[\rightarrow \mathbb{R}: p \in W^{1, \infty}([0, \infty)), p^{\prime}(s)=0\right.\right.\right.$ for $s$ large enough $\}$ 
and

$$
\mathcal{P}^{+}:=\{p \in \mathcal{P}: p \geq 0\}
$$

Given any function $w$ and $a, b \in \mathbb{R}$ we shall use the notation $\{w \geq$ $a\}=\left\{x \in \mathbb{R}^{N}: w(x) \geq a\right\},\{a \leq w \leq b\}=\left\{x \in \mathbb{R}^{N}: a \leq w(x) \leq b\right\}$, and similarly for the sets $\{w>a\},\{w \leq a\},\{w<a\}$, etc. We denote by $w^{+}:=\max \{w, 0\}$, and by $w^{-}:=\min \{w, 0\}$. We consider the functions, $\operatorname{sign}_{0}$ and $\operatorname{sign}_{0}^{+}$, defined in $\mathbb{R}$ by

$$
\operatorname{sign}_{0}(r):=\left\{\begin{array}{ll}
1 & \text { if } r>0 \\
0 & \text { if } r=0 \\
-1 & \text { if } r<0,
\end{array} \quad \operatorname{sign}_{0}^{+}(r):= \begin{cases}1 & \text { if } r>0 \\
0 & \text { if } r \leq 0\end{cases}\right.
$$

We need to consider the following function space

$$
T B V_{\mathrm{r}}^{+}\left(\mathbb{R}^{N}\right):=\left\{w \in L^{1}\left(\mathbb{R}^{N}\right)^{+}: T_{a, b}^{a}(w) \in B V\left(\mathbb{R}^{N}\right), \forall T_{a, b} \in \mathcal{T}_{r}\right\},
$$

and to give a sense to the gradient $\nabla u$ of a function $u$ in the above function space. Notice that the above function spaces are closely related to the space $G B V\left(\mathbb{R}^{N}\right)$ of generalized functions of bounded variation introduced by E. De Giorgi and L. Ambrosio ([40], see also [1]). Using the chain rule for BV-functions (see for instance $[\mathbf{1}]$ ), with a similar proof to the one given in Lemma 2.1 of [13], we obtain the following result.

Lemma 3.5. For every $w \in T B V_{\mathrm{r}}^{+}\left(\mathbb{R}^{N}\right)$ there exists a unique measurable function $v: \mathbb{R}^{N} \rightarrow \mathbb{R}^{N}$ such that

$$
\nabla T_{a, b}(w)=v \chi_{\{a<w<b\}} \quad \mathcal{L}^{N} \text {-a.e., } \forall T_{a, b} \in \mathcal{T}_{r}
$$

Thanks to this result we define $\nabla w$ for a function $w \in T B V_{\mathrm{r}}^{+}\left(\mathbb{R}^{N}\right)$ as the unique function $v$ which satisfies (3.11). This notation will be used throughout in the sequel.

Similar to the Lemma 3.4 in [5] we have the following result.

Lemma 3.6. If $w \in T B V_{\mathrm{r}}^{+}\left(\mathbb{R}^{N}\right)$, then $p(w) \in B V\left(\mathbb{R}^{N}\right)$ for every $p \in \mathcal{P}$ such that there exists $a>0$ with $p(r)=0$ for all $0 \leq r \leq a$. Moreover, we have $\nabla p(w)=p^{\prime}(w) \nabla w \quad \mathcal{L}^{N}$-a.e. .

By a direct application of Lemma 3.6, we have: 
Lemma 3.7. Let $S, T \in \mathcal{P}$. Then $S T, J_{T^{\prime} S}, J_{T S^{\prime}} \in \mathcal{P}$, where for any function $q, J_{q}(r)$ denotes the primitive of q, i.e., $J_{q}(r)=\int_{0}^{r} q(s) d s$. If one of the functions $S, T$ is such that it vanishes in $[0, a]$ for some $a>0$, then this holds also for $S T, J_{T^{\prime} S}, J_{T S^{\prime}}$. Therefore, if $w \in T B V_{\mathrm{r}}^{+}\left(\mathbb{R}^{N}\right)$ and there exists $a>0$ with $p(r)=0$ for all $0 \leq r \leq a$ for $p=S$ or $T$, then $S T(w), J_{T^{\prime} S}(w), J_{T S^{\prime}}(w) \in B V\left(\mathbb{R}^{N}\right)$. Moreover, we have

$$
D(S(w) T(w))=D J_{T^{\prime} S}(w)+D J_{T S^{\prime}}(w) .
$$

Hence, if $\mathbf{z} \in X_{1}\left(\mathbb{R}^{N}\right)$, we have

$$
(\mathbf{z} \cdot D(S(w) T(w)))=\left(\mathbf{z} \cdot D J_{T^{\prime} S}(w)\right)+\left(\mathbf{z} \cdot D J_{T S^{\prime}}(w)\right),
$$

where the equality is understood in $\mathcal{D}^{\prime}\left(\mathbb{R}^{N}\right)$, and as identity of Radon measures in $\mathbb{R}^{N}$.

Let $g: \mathbb{R}^{N} \times \mathbb{R} \times \mathbb{R}^{N} \rightarrow[0, \infty[$ be a function satisfying the assumptions of Theorem 2.1, and $T=T_{a, b} \in \mathcal{T}_{r}$ where $0<a<b$. Let us consider the functional

$$
R(g, T)(w):=\int_{\mathbb{R}^{N}} g(x, w(x), \nabla T(w(x))) d x, \quad w \in W^{1,1}\left(\mathbb{R}^{N}\right) .
$$

Assume that

$$
\begin{aligned}
& \chi_{\{w \leq a\}}(g(x, w(x), 0)-g(x, a, 0)), \\
& \chi_{\{w \geq b\}}(g(x, u(x), 0)-g(x, b, 0)) \in L^{1}\left(\mathbb{R}^{N}\right)
\end{aligned}
$$

for any $w \in L^{1}\left(\mathbb{R}^{N}\right)^{+}$. For $w \in T B V_{\mathrm{r}}^{+}\left(\mathbb{R}^{N}\right)$, if we define

$$
\begin{aligned}
\mathcal{R}\left(g, T_{a, b}\right)(w):= & \mathcal{R}_{g}\left(T_{a, b}(w)\right) \\
& +\int_{\{w \leq a\}}(g(x, w(x), 0)-g(x, a, 0)) d x \\
& +\int_{\{w \geq b\}}(g(x, w(x), 0)-g(x, b, 0)) d x,
\end{aligned}
$$

by Theorem 2.1 we have that $\mathcal{R}\left(g, T_{a, b}\right)$ is lower semi-continuous in $T B V_{\mathrm{r}}^{+}\left(\mathbb{R}^{N}\right)$ with respect to the $L^{1}\left(\mathbb{R}^{N}\right)$-convergence. Moreover, if $w \in$ 
$W^{1,1}\left(\mathbb{R}^{N}\right)$, we have the consistency with $R(g, T)(w)$ :

$$
\begin{aligned}
R(g, T)(w)= & \int_{\mathbb{R}^{N}} g(x, w(x), \nabla T(w)(x)) d x \\
= & \int_{\{a<w<b\}} g(x, w(x), \nabla w(x)) d x \\
& +\int_{\{w \leq a\}} g(x, w(x), 0) d x+\int_{\{w \geq b\}} g(x, w(x), 0) d x \\
= & \int_{\{a<w<b\}} g(x, w(x), \nabla w(x)) d x \\
& +\int_{\{w \leq a\}} g(x, a, 0) d x+\int_{\{w \geq b\}} g(x, b, 0) d x \\
& +\int_{\{w<a\}}(g(x, w(x), 0)-g(x, a, 0)) d x \\
& +\int_{\{w \geq b\}}(g(x, w(x), 0)-g(x, b, 0)) d x \\
= & \mathcal{R}_{g}\left(T_{a, b}(w)\right)+\int_{\{w<a\}}(g(x, w(x), 0)-g(x, a, 0)) d x \\
& +\int_{\{w \geq b\}]}(g(x, w(x), 0)-g(x, b, 0)) d x=\mathcal{R}(g, T)(w) .
\end{aligned}
$$

Since it will be sufficient for our purposes let us assume that $g$ does not depend on $x$. If $w \in T B V_{\mathrm{r}}^{+}\left(\mathbb{R}^{N}\right)$ and $T \in \mathcal{T}_{r}$, we define the Radon measure $g(w, D T(w))$ in $\mathbb{R}^{N}$ by

$$
\langle g(w, D T(w)), \phi\rangle:=\mathcal{R}(\phi g, T)(w),
$$

for all $\phi \in C_{c}\left(\mathbb{R}^{N}\right)$. Using (3.14), (3.15), and (2.4) we observe that

$$
\begin{aligned}
\langle g(w, D T(w)), \phi\rangle= & \langle g(T(w), D T(w)), \phi\rangle \\
& +\int_{\{w \leq a\}} \phi(g(x, w(x), 0)-g(x, a, 0)) d x \\
& +\int_{\{w \geq b\}} \phi(g(x, w(x), 0)-g(x, b, 0)) d x .
\end{aligned}
$$

3.2.2. Motivation of the functional calculus. Let us give the motivation for the functional calculus. For that, let us consider the problem (3.1) where $v \in\left(L^{1}\left(\mathbb{R}^{N}\right) \cap L^{\infty}\left(\mathbb{R}^{N}\right)\right)^{+}$. By the results in [5], [6] one can expect that $u \in\left(L^{1}\left(\mathbb{R}^{N}\right) \cap L^{\infty}\left(\mathbb{R}^{N}\right)\right)^{+}$and $\mathbf{a}(u, \nabla \Phi(u)) \in$ $L^{\infty}\left(\mathbb{R}^{N}, \mathbb{R}^{N}\right)$ (also in $L^{1}\left(\mathbb{R}^{N}, \mathbb{R}^{N}\right)$ since we assume that $|\mathbf{a}(u, \nabla \Phi(u))| \leq$ $\left.M u^{\bar{m}}, \bar{m} \geq 1\right)$. Moreover we have that $T_{a, b}^{a}(\Phi(u)) \in B V\left(\mathbb{R}^{N}\right)$ for any 
$0<a<b$. To prove uniqueness using the Kružkov's method [42], [28] we need to use a test function $T_{a, b}^{a}(\Phi(u))$ with $0<a<b$ so that $T_{a, b}^{a}(\Phi(u)) \in B V\left(\mathbb{R}^{N}\right)$, and a test function $S(\Phi(u)) \geq 0$ to approximate the sign ${ }^{+}$function. Let us call these test functions $T(\Phi(u))$ and $S(\Phi(u))$, respectively. For simplicity, let us write

$$
w=\Phi(u) .
$$

Multiplying (3.1) by $S(w) T(w)$ and integrating by parts we get

$$
\int_{\mathbb{R}^{N}} u+\int_{\mathbb{R}^{N}} S(w) \mathbf{a}(u, \nabla w) D T(w)+\int_{\mathbb{R}^{N}} T(w) \mathbf{a}(u, \nabla w) D S(w)=\int_{\mathbb{R}^{N}} v
$$

We have to give a sense to the integrals containing $\mathbf{a}(u, \nabla w)$. Let us analyze one of them, for instance the first

$$
\int_{\mathbb{R}^{N}} S(w) \mathbf{a}(u, \nabla w) D T(w) .
$$

Assume first that $T(\bar{z})=\bar{z}, \bar{z} \in \mathbb{R}$. In order to use the measures introduced in Subsection 2.2, we define $h(z, \xi)=\mathbf{a}(z, \xi) \cdot \xi, \mathbf{a}^{\Phi}(\bar{z}, \xi)=$ $\mathbf{a}\left(\Phi^{-1}(\bar{z}), \xi\right)$ and $h^{\Phi}(\bar{z}, \xi)=h\left(\Phi^{-1}(\bar{z}), \xi\right)=\mathbf{a}\left(\Phi^{-1}(\bar{z}), \xi\right) \cdot \xi=\mathbf{a}^{\Phi}(\bar{z}, \xi) \cdot \xi$. Then we define

$$
h(u, D w):=h^{\Phi}(w, D w) .
$$

Notice that we identify $z=u, \bar{z}=w, \xi=\nabla w$. Then we interpret (3.17) as

$$
\int_{\mathbb{R}^{N}} h_{S}^{\Phi}(w, D w)
$$

where $h_{S}^{\Phi}(\bar{z}, \xi)=S(\bar{z}) h^{\Phi}(\bar{z}, \xi)$. If $T(w)=T_{a, b}(w)-a$ with $0<a<b$, then we observe that

$$
\begin{aligned}
\mathbf{a}(u, \nabla w) \cdot \nabla T(w) & =\mathbf{a}(u, \nabla T(w)) \cdot \nabla T(w)=\mathbf{a}^{\Phi}(w, \nabla T(w)) \cdot \nabla T(w) \\
& =\mathbf{a}^{\Phi}\left(T_{a, b}(w), \nabla T(w)\right) \cdot \nabla T(w),
\end{aligned}
$$

and we interpret (3.17) as

$$
\int_{\mathbb{R}^{N}} h_{S}^{\Phi}(w, D T(w)) .
$$

These test functions are sufficient to prove uniqueness of entropy solutions. But, as in [30], for several reasons we need to consider a slightly more general class of test functions. The first reason is to define the notions of entropy sub- and supersolutions and its connection with the notion of entropy solution. The second comes from the work [30] where we exploited them in order to clarify the meaning of the entropy conditions on the jump sets. In order to study the full power of this functional calculus let us consider test functions of the form $T(\bar{z})=\tilde{T}\left(T_{a, b}(\bar{z})\right)$ where 
$\tilde{T}$ is differentiable in a neighborhood of $[a, b]$. In that case we formally write

$$
\begin{aligned}
S(w) \mathbf{a}(u, \nabla w) D T(w) & =S(w) \tilde{T}^{\prime}\left(T_{a, b}(w)\right) \mathbf{a}(u, \nabla w) D T_{a, b}(w) \\
& =S(w) \tilde{T}^{\prime}(w) \mathbf{a}\left(u, \nabla T_{a, b}(w)\right) D T_{a, b}(w) \\
& =S(w) \tilde{T}^{\prime}(w) \mathbf{a}^{\Phi}\left(w, \nabla T_{a, b}(w)\right) D T_{a, b}(w) .
\end{aligned}
$$

Then we define

$$
h_{S: T}^{\Phi}(\bar{z}, \xi)=S(\bar{z}) \tilde{T}^{\prime}(\bar{z}) h^{\Phi}(\bar{z}, \xi)
$$

and we interpret (3.17) as

$$
\int_{\mathbb{R}^{N}} h_{S: T}^{\Phi}\left(w, D T_{a, b}(w)\right) .
$$

This is the type of functional calculus that we need. This coincides with the functional calculus used in [30] applied to the function $w=\Phi(u)$ and writing $\mathbf{a}^{\Phi}, h^{\Phi}$ instead of $\mathbf{a}$ and $h$.

3.2.3. A functional calculus. Let us define the functional calculus. It represents an extension of the functional calculus in [5], [6], [29]. Let $S \in C([0, \infty))$ and $p \in \mathcal{P} \cap C^{1}([0, \infty))$. We denote

$$
\begin{array}{ll}
f^{\Phi}(\bar{z}, \xi)=f\left(\Phi^{-1}(\bar{z}), \xi\right), & h^{\Phi}(\bar{z}, \xi)=h\left(\Phi^{-1}(\bar{z}), \xi\right), \\
f_{S: p}^{\Phi}(\bar{z}, \xi)=S(\bar{z}) p^{\prime}(\bar{z}) f^{\Phi}(\bar{z}, \xi), & h_{S: p}^{\Phi}(\bar{z}, \xi)=S(\bar{z}) p^{\prime}(\bar{z}) h^{\Phi}(\bar{z}, \xi) .
\end{array}
$$

If $S p^{\prime} \geq 0$, then the function $f_{S: p}^{\Phi}(\bar{z}, \xi)$ satisfies the assumptions of Theorem 2.1 .

Assume that $p(\bar{z})=p\left(T_{a, b}(\bar{z})\right), 0<a<b$. We assume that $\Phi(u) \in$ $T B V_{\mathrm{r}}^{+}\left(\mathbb{R}^{N}\right)$ and

$$
\begin{aligned}
& \chi_{\{u \leq a\}} S(\Phi(u(x)))(f(u(x), 0)-f(a, 0)), \\
& \chi_{\{u \geq b\}} S(\Phi(u(x)))(f(u(x), 0)-f(b, 0)) \in L^{1}\left(\mathbb{R}^{N}\right) .
\end{aligned}
$$

Since $h(z, 0)=0$, this assumption holds for $h$.

For simplicity, we use again the notation $w=\Phi(u)$. We denote by

$$
f_{S: p}^{\Phi}\left(w, D T_{a, b}(w)\right), \quad h_{S: p}^{\Phi}\left(w, D T_{a, b}(w)\right),
$$

or simply by

$$
f_{S \circ \Phi: p \circ \Phi}\left(u, D T_{a, b}(w)\right), \quad h_{S \circ \Phi: p \circ \Phi}\left(u, D T_{a, b}(w)\right),
$$

the Radon measures defined by (3.15) with $g(\bar{z}, \xi)=f_{S: p}^{\Phi}(\bar{z}, \xi)$, and $g(\bar{z}, \xi)=h_{S: p}^{\Phi}(\bar{z}, \xi)$ applied to $w$, respectively. 
The above definitions can be extended to any $p \in \mathcal{P}$ such that $p(\bar{z})=$ $\tilde{p}\left(T_{a, b}(\bar{z})\right)$ and $\tilde{p}$ is differentiable in a neighborhood of $[a, b]$ (frequently we shall forget the distinction between $p$ and $\tilde{p}$ ) if we write

$$
f_{S: p}^{\Phi}(\bar{z}, \xi)=S(\bar{z}) \tilde{p}^{\prime}(\bar{z}) f^{\Phi}(\bar{z}, \xi), \quad h_{S: p}^{\Phi}(\bar{z}, \xi)=S(\bar{z}) \tilde{p}^{\prime}(\bar{z}) h^{\Phi}(\bar{z}, \xi) .
$$

In particular, if we take $T(\bar{z})=T_{a, b}(\bar{z})+c, 0 \leq a<b, c \in \mathbb{R}$, then we may write $T(\bar{z})=\tilde{p}\left(T_{a, b}(\bar{z})\right)$ where $\tilde{p} \in \mathcal{P}$ is given by $\tilde{p}(\bar{z})=\bar{z}+c$ for any $\bar{z} \in \mathbb{R}$. In that case, let

$f_{S}^{\Phi}(\bar{z}, \xi)=S(\bar{z}) f^{\Phi}(\bar{z}, \xi), \quad h_{S}^{\Phi}(\bar{z}, \xi)=S(\bar{z}) h^{\Phi}(\bar{z}, \xi) \quad \forall \bar{z} \in[0, \infty), \forall \xi \in \mathbb{R}^{N}$,

and define

$$
\begin{aligned}
& f_{S}^{\Phi}(w, D T(w)):=f_{S}^{\Phi}\left(w, D T_{a, b}(w)\right) \quad \text { and } \\
& h_{S}^{\Phi}(w, D T(w)):=h_{S}^{\Phi}\left(w, D T_{a, b}(w)\right) .
\end{aligned}
$$

Notice that, when $S \in \mathcal{T}^{+}, \Phi(z)=z, f_{S}(u, D T(u))$ and $h_{S}(u, D T(u))$ coincide with the definitions in [5], [6].

Since $h(z, 0)=0$ for all $z \in \mathbb{R}$, if $S \in C([0, \infty)), T \in \mathcal{T}^{+}$, with $T=T_{a, b}+c, c \in \mathbb{R}$, we have

$$
h_{S}^{\Phi}(w, D T(w))=h_{S}^{\Phi}\left(T_{a, b}(w), D T(w)\right)=h_{S}^{\Phi}\left(T_{a, b}(w), D T_{a, b}(w)\right) .
$$

Let $S \in C([0, \infty))$ and $p \in \mathcal{P}$ such that $p(r)=\tilde{p}\left(T_{a, b}(r)\right)$ and $\tilde{p}$ is differentiable in a neighborhood of $[a, b]$. Let $\varepsilon=+1$ if $S p^{\prime} \geq 0$ and $\varepsilon=-1$ if $S p^{\prime} \leq 0$. Taking this and (2.6) into account, and considering only the cases $S p^{\prime} \geq 0$ or $S p^{\prime} \leq 0$, we have

$$
\begin{aligned}
\left(f_{S: p}^{\Phi}\left(w, D T_{a, b}(w)\right)\right)^{s} & =\left(f_{S: p}^{\Phi}\left(T_{a, b}(w), D T_{a, b}(w)\right)\right)^{s} \\
& =\varepsilon \psi^{0}\left(\frac{D T_{a, b}(w)}{\left|D T_{a, b}(w)\right|}\right)\left|D^{s} J_{S p^{\prime} \varphi^{\Phi}}\left(T_{a, b}(w)\right)\right|
\end{aligned}
$$

since $f^{\Phi}(\bar{z}, \xi)=\varphi^{\Phi}(\bar{z}) \psi^{0}(\xi)$. Similarly, we have

$$
\begin{aligned}
\left(h_{S: p}^{\Phi}\left(w, D T_{a, b}(w)\right)\right)^{s} & =\left(h_{S: p}^{\Phi}\left(T_{a, b}(w), D T_{a, b}(w)\right)\right)^{s} \\
& =\varepsilon \psi^{0}\left(\frac{D T_{a, b}(w)}{\left|D T_{a, b}(w)\right|}\right)\left|D^{s} J_{S p^{\prime} \varphi^{\Phi}}\left(T_{a, b}(w)\right)\right| .
\end{aligned}
$$

Note that both singular parts are identical. By the representation formulas in Subsection 2.2, the absolutely continuous part of $h_{S: p}^{\Phi}\left(w, D T_{a, b}(w)\right)$ is $S(w) p^{\prime}(w) h\left(u, \nabla T_{a, b}(w)\right)$. Similar identities are true when $S=1$.

Remark 3.8. The functional calculus based on $f^{\Phi}(T(w), D T(w))$ with $w=\Phi(u), T=T_{a, b}$, is the same as the functional calculus based on $f\left(\widetilde{T}(u), \Phi^{\prime}(\widetilde{T}(u)) D \widetilde{T}(u)\right)$, where $\widetilde{T}=T_{\Phi^{-1}(a), \Phi^{-1}(b)}$, assuming $\Phi, \Phi^{-1}$ 
Lispchitz and $\Phi^{\prime}$ continuous in $[a, b]$ for any $0<a<b$. Avoiding technical details, this can be seen if we consider the functional calculus as the limit of integrals $\int_{\mathbb{R}^{N}} f^{\Phi}\left(w_{n}, D w_{n}\right)$ and $\int_{\mathbb{R}^{N}} f\left(u_{n}, \Phi^{\prime}\left(u_{n}\right) D u_{n}\right)$ where the functions $w_{n} \rightarrow T(w)$ and $u_{n} \rightarrow \widetilde{T}(u)$ in $L_{\text {loc }}^{1}\left(\mathbb{R}^{N}\right), u_{n}, w_{n} \in W^{1,1}\left(\mathbb{R}^{N}\right)$, noticing that the change of variables $w_{n}=\Phi\left(u_{n}\right)$ permits to identify both integrals.

3.3. The definition of entropy solution of (3.1). In order to introduce the concept of entropy solution for problem (3.1), let us discuss on the set of tests functions required for the entropy inequalities. We follow the presentation in $[\mathbf{2 9}]$.

The entropy inequalities may be classified into two groups: the entropy inequalities for subsolutions and for supersolutions. While the test functions for subsolutions should guarantee that $u-\operatorname{div} \mathbf{a}(u, \nabla \Phi(u)) \leq$ $v$ for the elliptic problem or $u_{t}-\operatorname{div} \mathbf{a}(u, \nabla \Phi(u)) \leq 0$ for the parabolic one, the test functions for supersolutions should imply that $u-$ $\operatorname{div} \mathbf{a}(u, \nabla \Phi(u)) \geq v, u_{t}-\operatorname{div} \mathbf{a}(u, \nabla \Phi(u)) \geq 0$, respectively. On the other hand, due to the form in which they appear in (3.19) in Definition 3.10 or Definition 5.1, we need to use the lower semicontinuity result of Theorem 2.1 in order to prove them. This requires that we assume that the product $S T$ is a non-decreasing function and that both terms $S^{\prime} T, S T^{\prime} \geq 0$.

Then the test functions for entropy subsolutions should satisfy:

Sub(i): $S \geq 0, S^{\prime} \geq 0$ and $T \geq 0, T^{\prime} \geq 0$.

Sub(ii): $S \leq 0, S^{\prime} \leq 0$ and $T \leq 0, T^{\prime} \leq 0$.

The test functions for entropy supersolutions should satisfy:

Super(i): $S \leq 0, S^{\prime} \geq 0$ and $T \geq 0, T^{\prime} \leq 0$.

Super(ii): $S \geq 0, S^{\prime} \leq 0$ and $T \leq 0, T^{\prime} \geq 0$.

Notice that the transformation $(S, T) \rightarrow(-S,-T)$ maps the case Sub(ii) into Sub(i) and Super(ii) into Super(i). Thus, it will be sufficient that the entropy inequalities for subsolution (resp. supersolution) are satisfied in the case Sub(i) (resp. Super(i)).

Remark 3.9. By changing the inequalities $\leq$ into $\geq$ in (3.19) in Definition 3.10 or Definition 5.1, the entropy conditions could also be written assuming that $S T$ is a non-increasing function and that both terms $S^{\prime} T, S T^{\prime} \leq 0$. This amounts to change $S \rightarrow-S$ in the condition above.

Let us give a notation for the class of test functions required to define entropy subsolutions. If $w:=\Phi(u) \in T B V_{\mathrm{r}}^{+}\left(\mathbb{R}^{N}\right)$, we define $\mathcal{T} \mathcal{S U B}$ the class of functions $S, T \in \mathcal{P}$ satisfying $\operatorname{Sub}(\mathrm{i})$ and $p(\bar{z})=\tilde{p}\left(T_{a, b}(\bar{z})\right)$ 
for some $0<a<b$ where $\tilde{p}$ is differentiable in a neighborhood of $[a, b]$ and $p$ represents either $S$ or $T$. The class of test functions for supersolutions can be defined in a similar way. We denote it by $\mathcal{T S U P \mathcal { E R }}$.

Although the proof of uniqueness and the development of the theory requires only the use of test functions $S, T \in \mathcal{T}^{+}$and this was the family used in $[\mathbf{5}],[\mathbf{6}]$, the analysis of the entropy conditions is facilitated if we use test functions with the signs considered in $\mathcal{T S U B}$.

Definition 3.10. Given $v \in L^{1}\left(\mathbb{R}^{N}\right) \cap L^{\infty}\left(\mathbb{R}^{N}\right), v \geq 0$, we say that $u \geq 0$ is an entropy solution of (3.1) if $u \in T B V_{r}^{+}\left(\mathbb{R}^{N}\right) \cap L^{\infty}\left(\mathbb{R}^{N}\right)$ and $\mathbf{a}(u, \nabla \Phi(u))$ is a vector field in $X_{1}\left(\mathbb{R}^{N}, \mathbb{R}^{N}\right)$ satisfying

$$
\begin{gathered}
u-\operatorname{div} \mathbf{a}(u, \nabla \Phi(u)))=v \quad \text { in } \quad \mathcal{D}^{\prime}\left(\mathbb{R}^{N}\right), \\
h_{S: T}^{\Phi}\left(\Phi(u), D T_{a, b}(\Phi(u))\right) \leq\left(\mathbf{a}(u, \nabla \Phi(u)), D J_{T^{\prime} S}(\Phi(u))\right) \\
\quad \text { as measures } \forall(S, T) \in \mathcal{T} \mathcal{S} \mathcal{B} .
\end{gathered}
$$

Inequality (3.19) holds in the sense of distributions. Since $h_{S: T}^{\Phi}(\Phi(u)$, $\left.D T_{a, b}(\Phi(u))\right)$ is a Radon measure, then $\left(\mathbf{a}(u, \nabla \Phi(u)), D J_{T^{\prime} S}(\Phi(u))\right)$ is also a Radon measure and (3.19) holds in the sense of measures. We notice that the analysis of the Radon measure $\left(\mathbf{a}(u, \nabla \Phi(u)), D J_{T^{\prime} S}(\Phi(u))\right)$ is easy when $v \in\left(L^{1}\left(\mathbb{R}^{N}\right) \cap L^{\infty}\left(\mathbb{R}^{N}\right)\right)^{+}$since $\mathbf{a}(u, \nabla \Phi(u)) \in X_{1}\left(\mathbb{R}^{N}\right)$. In particular, its absolutely continuous part is $\mathbf{a}(u, \nabla \Phi(u)) \cdot \nabla J_{T^{\prime} S}(\Phi(u))$ since $\left(\mathbf{a}(u, \nabla \Phi(u)), D J_{T^{\prime} S}(\Phi(u))\right)^{s}$ is absolutely continuous with respect to $D J_{T^{\prime} S}(\Phi(u))^{s}[\mathbf{1 0}]$.

Remark 3.11. The notion of entropy subsolution of (3.1) can be defined by replacing the identity in (3.18) by the inequality $\leq$. To define the notion of entropy supersolution of (3.1) we have to replace the identity in (3.18) by the inequality $\geq$ and use test functions $(S, T) \in \mathcal{T} \mathcal{S U P \mathcal { E R }}$ in (3.19).

3.4. An existence and uniqueness result for the elliptic problem. The main result of this section is the following existence and uniqueness result.

Theorem 3.12. Assume that assumptions $(\mathrm{H})$ hold. Then, for any $0 \leq v \in L^{1}\left(\mathbb{R}^{N}\right) \cap L^{\infty}\left(\mathbb{R}^{N}\right)$ there exists a unique entropy solution $u \in$ $L^{1}\left(\mathbb{R}^{N}\right) \cap L^{\infty}\left(\mathbb{R}^{N}\right)$ such that $u \in T B V_{\mathrm{r}}^{+}\left(\mathbb{R}^{N}\right)$ of the problem

$$
u-\operatorname{div} \mathbf{a}(u, D \Phi(u))=v \quad \text { in } \mathbb{R}^{N} .
$$

Moreover, given $v, \bar{v} \in\left(L^{1}\left(\mathbb{R}^{N}\right) \cap L^{\infty}\left(\mathbb{R}^{N}\right)\right)^{+}$, if $u, \bar{u}$ are entropy solutions of the problems

$$
u-\operatorname{div} \mathbf{a}(u, D \Phi(u))=v \quad \text { in } \quad \mathbb{R}^{N}
$$


and

$$
\bar{u}-\operatorname{div} \mathbf{a}(\bar{u}, D \Phi(\bar{u}))=\bar{v} \quad \text { in } \quad \mathbb{R}^{N}
$$

respectively, then

$$
\int_{\mathbb{R}^{N}}(u-\bar{u})^{+} \leq \int_{\mathbb{R}^{N}}(v-\bar{v})^{+} .
$$

The proof follows the steps of the analogous proof in [5] (see also [29]). We give it in detail so that we can skip the proof of the parabolic case, that is also based on the analogous results in $[\mathbf{6}],[\mathbf{2 9}]$ with similar adaptations.

\subsubsection{The proof of existence of entropy solutions for the elliptic} problem. We divide the proof in different steps.

Step 1. Approximation and basic estimates. Let $0 \leq v \in L^{\infty}\left(\mathbb{R}^{N}\right) \cap$ $L^{1}\left(\mathbb{R}^{N}\right)$. As a consequence of the results about pseudomonotone operators given in $[\mathbf{2 6}]$ we know that for any $n \in \mathbb{N}$ there exists $u_{n} \in$ $W^{1,2}\left(\mathbb{R}^{N}\right) \cap L^{\infty}\left(\mathbb{R}^{N}\right), u_{n} \geq 0$, such that

$$
u_{n}-\operatorname{div} \mathbf{a}\left(u_{n}, \nabla \Phi\left(u_{n}\right)\right)-\frac{1}{n} \Delta u_{n}=v \quad \text { in } \mathcal{D}^{\prime}\left(\mathbb{R}^{N}\right) .
$$

Let $p \in \mathcal{P}_{0}$. Multiplying (3.22) by $p\left(u_{n}\right)$ and integrating by parts, we obtain

$$
\int_{\mathbb{R}^{N}} u_{n} p\left(u_{n}\right) \leq \int_{\mathbb{R}^{N}} v p\left(u_{n}\right) .
$$

Then, by Proposition 2.2, it follows that $u_{n} \ll v$ for all $n \in \mathbb{N}$, and consequently, we have

$$
\left\|u_{n}\right\|_{p} \leq\|v\|_{p} \quad \text { for all } n \in \mathbb{N}, \quad \text { for all } p \in[1, \infty] .
$$

Since we assume that $v \in L^{\infty}\left(\mathbb{R}^{N}\right)$, then $u_{n} \in L^{\infty}\left(\mathbb{R}^{N}\right)$. Moreover, $u_{n} \geq 0$, and by Proposition 2.2 we have that

(3.25) $\left\{u_{n}: n \in \mathbb{N}\right\}$ is a weakly sequentially compact subset of $L_{0}\left(\mathbb{R}^{N}\right)$.

Let $0<a<b$. Multiply $(3.22)$ by $T_{a, b}\left(\Phi\left(u_{n}\right)\right)-a$. Since $\mathbf{a}\left(u_{n}, \nabla \Phi\left(u_{n}\right)\right)+$ $\frac{1}{n} \nabla u_{n} \in L^{2}\left(\mathbb{R}^{N}\right)$, and $\operatorname{div}\left(\mathbf{a}\left(u_{n}, \nabla \Phi\left(u_{n}\right)\right)+\frac{1}{n} \nabla u_{n}\right) \in L^{1}\left(\mathbb{R}^{N}\right)$, after integration by parts we obtain

$$
\begin{aligned}
\int_{\mathbb{R}^{N}} u_{n}\left(T_{a, b}\left(\Phi\left(u_{n}\right)\right)-a\right) & +\int_{\mathbb{R}^{N}} \mathbf{a}\left(u_{n}, \nabla \Phi\left(u_{n}\right)\right) \nabla T_{a, b}\left(\Phi\left(u_{n}\right)\right) \\
& +\frac{1}{n} \int_{\mathbb{R}^{N}}\left|\nabla T_{\bar{a}, \bar{b}}\left(\Psi\left(u_{n}\right)\right)\right|^{2} \\
= & \int_{\mathbb{R}^{N}} v_{n}\left(T_{a, b}\left(\Phi\left(u_{n}\right)\right)-a\right)
\end{aligned}
$$


where $\Psi^{\prime}(z)=\sqrt{\Phi^{\prime}(z)}, \bar{a}=\Psi\left(\Phi^{-1}(a)\right), \bar{b}=\Psi\left(\Phi^{-1}(b)\right)$. Notice that $\Psi \in W^{1, \infty}([a, b])$ for all $0<a<b$. Since, by (3.8), we have

$a\left(u_{n}, \nabla \Phi\left(u_{n}\right)\right) \cdot \nabla T_{a, b}\left(\Phi\left(u_{n}\right)\right) \geq C_{0}\left(u_{n}\right)\left|\nabla T_{a, b}\left(\Phi\left(u_{n}\right)\right)\right|-D_{1}\left(u_{n}\right) T_{a, b}^{\prime}\left(\Phi\left(u_{n}\right)\right)$, using $\left(\mathrm{H}_{1}\right)$ and $\left(\mathrm{H}_{2}\right)$ we have that $D_{1}\left(u_{n}\right) T_{a, b}^{\prime}\left(\Phi\left(u_{n}\right)\right)$ is integrable and we obtain

$$
\begin{aligned}
& \inf _{z \in[a, b]} C_{0}(z) \int_{\mathbb{R}^{N}} \mid \nabla T_{a, b}\left(\Phi\left(u_{n}\right)\right) \mid d x \\
& \leq(b-a) \int_{\mathbb{R}^{N}} v+\int_{\mathbb{R}^{N}} D_{1}\left(u_{n}\right) T_{a, b}^{\prime}\left(\Phi\left(u_{n}\right)\right) \quad \forall n \in \mathbb{N}
\end{aligned}
$$

and

$$
\frac{1}{n} \int_{\mathbb{R}^{N}}\left|\nabla T_{\bar{a}, \bar{b}}\left(\Psi\left(u_{n}\right)\right)\right|^{2} d x \leq(b-a) \int_{\mathbb{R}^{N}} v \quad \forall n \in \mathbb{N} .
$$

By (3.25) and (3.24), by extracting a subsequence if is necessary, we may assume that $u_{n}$ converges weakly in $L_{0}\left(\mathbb{R}^{N}\right)$ and in $L^{2}\left(\mathbb{R}^{N}\right)$ to some nonnegative function $u$ as $n \rightarrow+\infty$. Moreover, by (3.24), we have that $0 \leq u \in L^{1}\left(\mathbb{R}^{N}\right) \cap L^{\infty}\left(\mathbb{R}^{N}\right)$. On the other hand, by extracting a subsequence, we may assume that $T_{a, b}\left(\Phi\left(u_{n}\right)\right)$ is a Cauchy sequence in $L_{\text {loc }}^{1}\left(\mathbb{R}^{N}\right)$ for all $a, b \in \mathbb{Q}, 0<a<b$. Let us prove that $\Phi\left(u_{n}\right)$ is locally a Cauchy sequence in measure. For that we adapt the strategy in [13]. Let $\delta>0, a, b \in \mathbb{Q}, 0<a<b$, with $\delta>2 a$ and $b>\sup _{n}\left\|u_{n}\right\|_{\infty}$. Let $B_{R}$ be the ball of radius $R>0$ in $\mathbb{R}^{N}$ centered at the origin. Then

$$
\begin{aligned}
\left\{\left|\Phi\left(u_{n}\right)-\Phi\left(u_{m}\right)\right|>\delta\right\} & \cap B_{R} \subseteq\left(\left\{\Phi\left(u_{n}\right)<a,\left|\Phi\left(u_{n}\right)-\Phi\left(u_{m}\right)\right|>\delta\right\} \cap B_{R}\right) \\
& \cup\left(\left\{\Phi\left(u_{m}\right)<a,\left|\Phi\left(u_{n}\right)-\Phi\left(u_{m}\right)\right|>\delta\right\} \cap B_{R}\right) \\
& \cup\left(\left\{\left|T_{a, b}\left(\Phi\left(u_{n}\right)\right)-T_{a, b}\left(\Phi\left(u_{m}\right)\right)\right|>\delta\right\} \cap B_{R}\right) \\
\subseteq & \left(\left\{\left|T_{a, b}\left(\Phi\left(u_{n}\right)\right)-T_{a, b}\left(\Phi\left(u_{m}\right)\right)\right|>\delta-a\right\} \cap B_{R}\right) \\
& \cup\left(\left\{\left|T_{a, b}\left(\Phi\left(u_{n}\right)\right)-T_{a, b}\left(\Phi\left(u_{m}\right)\right)\right|>\delta\right\} \cap B_{R}\right) \\
\subseteq & \left\{\left|T_{a, b}\left(\Phi\left(u_{n}\right)\right)-T_{a, b}\left(\Phi\left(u_{m}\right)\right)\right|>\delta-a\right\} \cap B_{R} .
\end{aligned}
$$

Since $T_{a, b}\left(\Phi\left(u_{n}\right)\right)$ is a Cauchy sequence in $L_{\text {loc }}^{1}\left(\mathbb{R}^{N}\right)$, then the measure of the set $\left\{\left|T_{a, b}\left(\Phi\left(u_{n}\right)\right)-T_{a, b}\left(\Phi\left(u_{m}\right)\right)\right|>\delta-a\right\} \cap B_{R}$ converges to zero. We deduce that $\Phi\left(u_{n}\right)$ is locally a Cauchy sequence in measure. In particular, by extracting a subsequence if necessary, we may assume that $\Phi\left(u_{n}\right)$ converges a.e. to some function $U \in L_{\text {loc }}^{1}\left(\mathbb{R}^{N}\right)$. By our assumptions on $\Phi, \Phi^{-1}$ is continuous in $[0, \infty)$ and we deduce that $u_{n}$ converges a.e. to $\Phi^{-1}(U)$. Clearly, $u=\Phi^{-1}(U)$. Then, by Proposition 2.2(iii) (or Vitali's Convergence Theorem), we get that $u_{n} \rightarrow u$ in $L^{1}\left(\mathbb{R}^{N}\right)$, in $L^{r}\left(\mathbb{R}^{N}\right)$ for all $r<\infty$, and $T_{a, b}\left(\Phi\left(u_{n}\right)\right) \rightarrow T_{a, b}(\Phi(u))$ in $L_{\text {loc }}^{1}\left(\mathbb{R}^{N}\right)$ for 
all $0<a<b$. Using the above estimate on the gradients we obtain that $\Phi(u) \in T B V_{r}^{+}\left(\mathbb{R}^{N}\right)$. Letting $n \rightarrow \infty$ in (3.23) we obtain that $u \ll v$. Since $\Phi^{-1} \in W^{1, \infty}([a, b])$ for all $0<a<b$, then $u \in T B V_{r}^{+}\left(\mathbb{R}^{N}\right)$.

Observe that by (3.7) we may assume that

$$
\begin{array}{r}
\mathbf{a}\left(u_{n}, \nabla \Phi\left(u_{n}\right)\right) \rightarrow \mathbf{z} \text { as } n \rightarrow \infty, \text { weakly in } L^{1}\left(\mathbb{R}^{N}, \mathbb{R}^{N}\right), \\
\text { and weakly* in } L^{\infty}\left(\mathbb{R}^{N}, \mathbb{R}^{N}\right) .
\end{array}
$$

Since, by assumption $\left(\mathrm{H}_{2}\right)$ we have that $\mathbf{a}\left(u_{n}, \nabla \Phi\left(u_{n}\right)\right)=u_{n}^{\bar{m}} \mathbf{b}\left(u_{n}, \nabla \Phi\left(u_{n}\right)\right)$ with $\left|\mathbf{b}\left(u_{n}, \nabla \Phi\left(u_{n}\right)\right)\right| \leq M$, and $\left\|u_{n}\right\|_{\infty} \leq\|v\|_{\infty}, u_{n} \rightarrow u$ a.e. and in $L^{r}\left(\mathbb{R}^{N}\right)$ for any $r \in[1, \infty)$ as $n \rightarrow \infty$, we may assume that

$$
\mathbf{b}\left(u_{n}, \nabla \Phi\left(u_{n}\right)\right) \rightarrow \mathbf{z}_{b} \quad \text { as } n \rightarrow \infty \text {, weakly* in } L^{\infty}\left(\mathbb{R}^{N}, \mathbb{R}^{N}\right),
$$

and

$$
\mathbf{z}=u^{\bar{m}} \mathbf{z}_{b} .
$$

Given $\phi \in \mathcal{D}\left(\mathbb{R}^{N}\right)$, multiplying (3.22) by $\phi$ and integrating by parts we obtain

$\int_{\mathbb{R}^{N}} \phi u_{n} d x+\int_{\mathbb{R}^{N}} \mathbf{a}\left(u_{n}, \nabla \Phi\left(u_{n}\right)\right) \cdot \nabla \phi d x+\frac{1}{n} \int_{\mathbb{R}^{N}} \nabla u_{n} \cdot \nabla \phi=\int_{\mathbb{R}^{N}} \phi v_{n} d x$.

Since

$$
\frac{1}{n} \int_{\mathbb{R}^{N}} \nabla u_{n} \cdot \nabla \phi=\frac{1}{n} \int_{\mathbb{R}^{N}} u_{n} \cdot \Delta \phi \rightarrow 0,
$$

letting $n \rightarrow+\infty$, we obtain

$$
\int_{\mathbb{R}^{N}} u \phi d x+\int_{\mathbb{R}^{N}} \mathbf{z} \cdot \nabla \phi d x=\int_{\mathbb{R}^{N}} v \phi d x,
$$

that is,

$$
u-\operatorname{div}(\mathbf{z})=v, \quad \text { in } \mathcal{D}^{\prime}\left(\mathbb{R}^{N}\right)
$$

and

(3.31) $\operatorname{div} \mathbf{a}\left(u_{n}, \nabla \Phi\left(u_{n}\right)\right)+\frac{1}{n} \Delta u_{n} \rightarrow \operatorname{div}(\mathbf{z}) \quad$ in $L^{r}\left(\mathbb{R}^{N}\right) \forall r \in[1, \infty)$.

Note that by (3.29) we have $\mathbf{z} \in L^{r}\left(\mathbb{R}^{N}\right)^{N} \forall r \in[1, \infty]$ and, by (3.30), $\operatorname{div}(\mathbf{z}) \in L^{r}\left(\mathbb{R}^{N}\right) \forall r \in[1, \infty]$.

Finally, letting $n \rightarrow \infty$ in (3.26) we have

(3.32) $\inf _{z \in[a, b]} C_{0}(z) \int_{\mathbb{R}^{N}}\left|\nabla T_{a, b}(\Phi(u))\right| d x \leq(b-a) \int_{\mathbb{R}^{N}} v+\int_{\mathbb{R}^{N}} D_{1}(u) T_{a, b}^{\prime}(\Phi(u))$.

Thus (again) $u \in T B V_{\mathrm{r}}^{+}\left(\mathbb{R}^{N}\right)$. 
Step 2. Identification of $\mathbf{z}(x)$.

Lemma 3.13. We have

$$
\mathbf{z}(x)=\mathbf{a}(u(x), \nabla \Phi(u(x))) \quad \text { a.e. } x \in \mathbb{R}^{N} .
$$

Proof: We use Minty-Browder's technique. Recall that $\nabla u_{n} \in L^{2}\left(\mathbb{R}^{N}\right)^{N}$ although the bounds are not uniform in $n$. Let $0<a<b$, let $0 \leq \phi \in$ $C_{c}^{1}\left(\mathbb{R}^{N}\right)$ and $g \in C^{2}\left(\mathbb{R}^{N}\right) \cap W^{1, \infty}\left(\mathbb{R}^{N}\right)$. Let us use the notation

$$
\mathbf{a}_{n}\left(u_{n}, \nabla \Phi\left(u_{n}\right)\right)=\mathbf{a}\left(u_{n}, \nabla \Phi\left(u_{n}\right)\right)+\frac{1}{n} \nabla u_{n} .
$$

For simplicity, let us write $w_{n}=\Phi\left(u_{n}\right), w=\Phi(u)$. By (3.6), we have

$$
\left.\int_{\mathbb{R}^{N}} \phi\left[\mathbf{a}\left(u_{n}, \nabla w_{n}\right)-\mathbf{a}\left(u_{n}, \nabla g\right)\right) \cdot \nabla\left(w_{n}-g\right)\right] T_{a, b}^{\prime}\left(w_{n}\right) d x \geq 0 .
$$

We use the notation $T_{a, b}^{\prime}$ to refer to $\chi_{(a, b)}$ and $\bar{T}_{a, b}^{\prime}$ to refer to $\chi_{[a, b]}$. Now, since

$$
\begin{aligned}
\int_{\mathbb{R}^{N}} \phi \mathbf{a} & \left(u_{n}, \nabla w_{n}\right) \cdot \nabla\left(w_{n}-g\right) T_{a, b}^{\prime}\left(w_{n}\right) d x \\
= & \int_{\mathbb{R}^{N}} \phi \mathbf{a}\left(u_{n}, \nabla w_{n}\right) \cdot \nabla\left(T_{a, b}\left(w_{n}\right)-g\right) d x \\
& +\int_{\mathbb{R}^{N}} \phi \mathbf{a}\left(u_{n}, \nabla w_{n}\right) \cdot \nabla g\left(1-T_{a, b}^{\prime}\left(w_{n}\right)\right) d x \\
= & \int_{\mathbb{R}^{N}} \phi \mathbf{a}_{n}\left(u_{n}, \nabla w_{n}\right) \cdot \nabla\left(T_{a, b}\left(w_{n}\right)-g\right) d x \\
& -\frac{1}{n} \int_{\mathbb{R}^{N}} \phi \nabla u_{n} \cdot \nabla\left(T_{a, b}\left(w_{n}\right)-g\right) d x \\
& +\int_{\mathbb{R}^{N}} \phi \mathbf{a}\left(u_{n}, \nabla w_{n}\right) \cdot \nabla g\left(1-T_{a, b}^{\prime}\left(w_{n}\right)\right) d x \\
\leq & -\int_{\mathbb{R}^{N}} \operatorname{div}\left(\mathbf{a}_{n}\left(u_{n}, \nabla w_{n}\right)\right) \phi\left(T_{a, b}\left(w_{n}\right)-g\right) d x \\
& -\int_{\mathbb{R}^{N}}\left(T_{a, b}\left(w_{n}\right)-g\right) \mathbf{a}\left(u_{n}, \nabla w_{n}\right) \cdot \nabla \phi d x, \\
& +\frac{1}{n} \int_{\mathbb{R}^{N}} \phi \nabla u_{n} \cdot \nabla g d x+\int_{\mathbb{R}^{N}} \phi \mathbf{a}\left(u_{n}, \nabla w_{n}\right) \cdot \nabla g\left(1-T_{a, b}^{\prime}\left(w_{n}\right)\right) d x,
\end{aligned}
$$

and

$$
\frac{1}{n} \int_{\mathbb{R}^{N}} \phi \nabla u_{n} \cdot \nabla g d x=\frac{1}{n} \int_{\mathbb{R}^{N}} u_{n} \operatorname{div}(\phi \nabla g) d x \rightarrow 0,
$$


we get

$$
\begin{aligned}
\lim _{n \rightarrow \infty} \int_{\mathbb{R}^{N}} \phi \mathbf{a}\left(u_{n}, \nabla w_{n}\right) \cdot \nabla\left(w_{n}-g\right) T_{a, b}^{\prime}\left(w_{n}\right) d x \\
\leq-\int_{\mathbb{R}^{N}} \operatorname{div}(\mathbf{z}) \phi\left(T_{a, b}(w)-g\right) d x-\int_{\mathbb{R}^{N}}\left(T_{a, b}(w)-g\right) \mathbf{z} \cdot \nabla \phi d x \\
\quad+M\|\nabla g\|_{\infty} \int_{\mathbb{R}^{N}} \phi u^{\bar{m}}\left(1-T_{a, b}^{\prime}(w)\right) d x \\
=\int_{\mathbb{R}^{N}} \phi\left(\mathbf{z} \cdot D\left(T_{a, b}(w)-g\right)\right)+M\|\nabla g\|_{\infty} \int_{\mathbb{R}^{N}} \phi u^{\bar{m}}\left(1-T_{a, b}^{\prime}(w)\right) d x .
\end{aligned}
$$

On the other hand, let us denote by

$J_{\mathbf{a}_{i}^{\Phi}}(x, r):=\int_{0}^{r} \mathbf{a}_{i}^{\Phi}(s, \nabla g(x)) d s, \quad$ and $\quad J_{\frac{\partial \mathbf{a}_{i}^{\Phi}}{\partial x_{j}}}(x, r):=\int_{0}^{r} \frac{\partial}{\partial x_{j}} \mathbf{a}_{i}^{\Phi}(s, \nabla g(x)) d s$,

$i, j \in\{1, \ldots, N\}$, and observe that

$$
\begin{aligned}
\frac{\partial}{\partial x_{j}} J_{\mathbf{a}_{i}^{\Phi}}\left(x, T_{a, b}\left(w_{n}(x)\right)\right)=\mathbf{a}_{i}^{\Phi}\left(w_{n}(x), \nabla g(x)\right) & \frac{\partial T_{a, b}\left(w_{n}\right)}{\partial x_{j}}(x) \\
& +J_{\frac{\partial \mathbf{a}_{i}^{\Phi}}{\partial x_{j}}}\left(x, T_{a, b}\left(w_{n}(x)\right)\right) .
\end{aligned}
$$

Now, since the right hand side of the above equality is bounded in $L^{1}\left(\mathbb{R}^{N}\right)$ and

$$
\frac{\partial}{\partial x_{j}} J_{\mathbf{a}_{i}^{\Phi}}\left(x, T_{a, b}\left(w_{n}\right)\right) \rightarrow \frac{\partial}{\partial x_{j}} J_{\mathbf{a}_{i}^{\Phi}}\left(x, T_{a, b}(w)\right)
$$

weakly as measures, and $J_{\frac{\partial \mathbf{a}_{i}^{\Phi}}{\partial x_{j}}}\left(x, T_{a, b}\left(w_{n}(x)\right)\right) \rightarrow J_{\frac{\partial \mathbf{a}_{i}^{\Phi}}{\partial x_{j}}}\left(x, T_{a, b}(w(x))\right)$ a.e., we have

$$
\begin{aligned}
& \lim _{n \rightarrow \infty} \int_{\mathbb{R}^{N}} \phi \mathbf{a}\left(u_{n}, \nabla g\right) \cdot \nabla\left(w_{n}-g\right) T_{a, b}^{\prime}\left(w_{n}\right) d x \\
&=\lim _{n \rightarrow \infty} \int_{\mathbb{R}^{N}} \phi \sum_{i=1}^{N}\left[\frac{\partial}{\partial x_{i}} J_{\mathbf{a}_{i}^{\Phi}}\left(x, T_{a, b}\left(w_{n}(x)\right)\right)-J_{\frac{\partial \mathbf{a}_{i}^{\Phi}}{\partial x_{i}}}\left(x, T_{a, b}\left(w_{n}(x)\right)\right)\right] \\
&-\lim _{n \rightarrow \infty} \int_{\mathbb{R}^{N}} \phi \mathbf{a}\left(u_{n}, \nabla g\right) \cdot \nabla g T_{a, b}^{\prime}\left(w_{n}\right) d x \\
& \geq \int_{\mathbb{R}^{N}} \phi \sum_{i=1}^{N}\left[\frac{\partial}{\partial x_{i}} J_{\mathbf{a}_{i}^{\Phi}}\left(x, T_{a, b}(w)\right)-J_{\frac{\partial \mathbf{a}_{i}^{\Phi}}{\partial x_{i}}}\left(x, T_{a, b}(w(x))\right)\right] \\
&-\int_{\mathbb{R}^{N}} \phi \mathbf{a}(u, \nabla g) \cdot \nabla g \bar{T}_{a, b}^{\prime}(w) d x .
\end{aligned}
$$


Hence we obtain

$$
\begin{aligned}
& \int_{\mathbb{R}^{N}} \phi\left(\mathbf{z} \cdot D\left(T_{a, b}(w)-g\right)\right)+M\|\nabla g\|_{\infty} \int_{\mathbb{R}^{N}} \phi u^{\bar{m}}\left(1-T_{a, b}^{\prime}(w)\right) d x \\
&+ \int_{\mathbb{R}^{N}} \phi \mathbf{a}(u, \nabla g) \cdot \nabla g \bar{T}_{a, b}^{\prime}(w) \\
&\left.\quad-\int_{\mathbb{R}^{N}} \phi\left(\sum_{i=1}^{N}\left[\frac{\partial}{\partial x_{i}} J_{\mathbf{a}_{i}^{\Phi}}\left(x, T_{a, b}(w(x))\right)-\frac{J_{\partial_{\mathbf{a}_{i}}^{\Phi}}\left(x, T_{i}\right.}{\partial x_{i} b}(w(x))\right)\right]\right) \geq 0
\end{aligned}
$$

for all $0 \leq \phi \in C_{c}^{1}\left(\mathbb{R}^{N}\right)$. Thus the measure

$$
\begin{gathered}
\left(\mathbf{z} \cdot D\left(T_{a, b}(w)-g\right)\right)-\sum_{i=1}^{N}\left[\frac{\partial}{\partial x_{i}} J_{\mathbf{a}_{i}^{\Phi}}\left(x, T_{a, b}(w(x))\right)-J_{\frac{\partial \mathbf{a}_{i}^{\Phi}}{\partial x_{i}}}\left(x, T_{a, b}(w(x))\right)\right] \\
+\mathbf{a}(u, \nabla g) \cdot \nabla g \bar{T}_{a, b}^{\prime}(w) \mathcal{L}^{N}+M\|\nabla g\|_{\infty} u^{m}\left(1-T_{a, b}^{\prime}(w)\right) \mathcal{L}^{N} \geq 0 .
\end{gathered}
$$

Then using chain's rule for $B V$ functions ([1, Theorem 3.96]) applied to $J_{\mathbf{a}_{i}}\left(u_{1}, u_{2}\right)$ with $u_{1}(x)=x, u_{2}(x)=T_{a, b}(w(x)), x \in \mathbb{R}^{N}$, we deduce that the absolutely continuous part of

$$
\sum_{i=1}^{N}\left[\frac{\partial}{\partial x_{i}} J_{\mathbf{a}_{i}^{\Phi}}\left(x, T_{a, b}(w(x))\right)-J_{\frac{\partial \mathbf{a}_{i}^{\Phi}}{\partial x_{i}}}\left(x, T_{a, b}(w(x))\right)\right]
$$

is $\mathbf{a}(u, \nabla g) \cdot \nabla T_{a, b}(w) \mathcal{L}^{N}$ and we obtain

$$
\begin{aligned}
\mathbf{z} \cdot \nabla\left(T_{a, b}(w)-g\right)-\mathbf{a}(u, \nabla g) \cdot \nabla & T_{a, b}(w)+\mathbf{a}(u, \nabla g) \cdot \nabla g \bar{T}_{a, b}^{\prime}(w) \\
& +M\|\nabla g\|_{\infty} u^{m}\left(1-T_{a, b}^{\prime}(w)\right) \geq 0 .
\end{aligned}
$$

In particular, for $x \in\{a<w<b\}$ we have

$$
(\mathbf{z}-\mathbf{a}(u, \nabla g)) \cdot \nabla(w-g) \geq 0 \quad \text { a.e. } .
$$

Since we may take a countable set of functions $g \in C^{2}\left(\mathbb{R}^{N}\right) \cap W^{1, \infty}\left(\mathbb{R}^{N}\right)$ dense in $C^{1}\left(\mathbb{R}^{N}\right)$ we have that the above inequality holds for all $x \in$ $\Omega \cap\{a<w<b\}$, where $\Omega \subset \mathbb{R}^{N}$ is such that $\mathcal{L}^{N}\left(\mathbb{R}^{N} \backslash \Omega\right)=0$, and all $g \in C^{1}\left(\mathbb{R}^{N}\right)$. Now, fixed $x \in \Omega \cap\{a<w<b\}$ and given $V \in \mathbb{R}^{N}$, there is $g \in C^{1}\left(\mathbb{R}^{N}\right)$ such that $\nabla g(x)=V$. Then

$$
(\mathbf{z}(x)-\mathbf{a}(u(x), V)) \cdot(\nabla w(x)-V) \geq 0, \quad \forall V \in \mathbb{R}^{N} .
$$

By an application of Minty-Browder's method in $\mathbb{R}^{N}$, these inequalities imply that

$$
\mathbf{z}(x)=\mathbf{a}(u(x), \nabla w(x)) \quad \text { a.e. on }\{a<w<b\} .
$$

Since this holds for any $0<a<b$, we obtain (3.33) a.e. on the points $x$ of $\mathbb{R}^{N}$ such that $\{w(x) \neq 0\}=\{u(x) \neq 0\}$. Now, by our assumptions on a 
and (3.29) we deduce that $\mathbf{z}(x)=\mathbf{a}(u(x), \nabla w(x))=0$ a.e. on $\{u=0\}$. We have proved (3.33).

From (3.33) and (3.30), it follows that

$$
u-\operatorname{div} \mathbf{a}(u, \nabla \Phi(u))=v, \quad \text { in } \mathcal{D}^{\prime}\left(\mathbb{R}^{N}\right) .
$$

Step 3. To finish the existence part of the proof we only need to prove that

Lemma 3.14. Let $(S, T) \in \mathcal{T} \mathcal{S U B} \cup \mathcal{T S U P \mathcal { R }}, T(\bar{z})=\tilde{T}\left(T_{a, b}(\bar{z})\right)$, $0<a<b$. Then

$$
h_{S: T}^{\Phi}\left(\Phi(u), D T_{a, b}(\Phi(u))\right) \leq\left(\mathbf{a}(u, \nabla \Phi(u)), D J_{T^{\prime} S}(\Phi(u))\right)
$$

as measures.

When writing $T(\bar{z})=\tilde{T}\left(T_{a, b}(\bar{z})\right)$ we assume that $\tilde{T}$ is differentiable in a neighborhood of $[a, b]$.

Since $h_{S: T}^{\Phi}\left(u, D T_{a, b}(\Phi(u))\right)$ is a Radon measure, it suffices to prove that the inequality (3.36) holds in $\mathcal{D}^{\prime}\left(\mathbb{R}^{N}\right)$. Thus, we use test functions $0 \leq \phi \in C_{c}^{2}\left(\mathbb{R}^{N}\right)$.

To prove (3.36) we require some intermediate inequalities summarized in the following lemmas.

Lemma 3.15. Let $(S, T) \in \mathcal{T S U B} \cup \mathcal{T S U P \mathcal { R }}$. We have the inequality

$$
\begin{aligned}
\limsup \int_{\mathbb{R}^{N}} \mathbf{a}\left(u_{n}, \nabla \Phi\left(u_{n}\right)\right) & \cdot \nabla\left(J_{T^{\prime} S}\left(\Phi\left(u_{n}\right)\right)\right) \phi(x) d x \\
\leq & \int_{\Omega} \phi\left(\mathbf{a}(u, \nabla \Phi(u)), D\left(J_{T^{\prime} S}(\Phi(u))\right)\right)
\end{aligned}
$$

for any $0 \leq \phi \in C_{c}^{2}\left(\mathbb{R}^{N}\right)$.

Lemma 3.16. Let $(S, T) \in \mathcal{T} \mathcal{S U B} \cup \mathcal{T S U P \mathcal { R }}$. Let $T(\bar{z})=\tilde{T}\left(T_{a, b}(\bar{z})\right)$. We have

$$
\begin{aligned}
& f_{S: T}^{\Phi}\left(\Phi(u), D T_{a, b}(\Phi(u))\right) \\
& \quad \leq\left(\mathbf{a}(u, \nabla \Phi(u)), D\left(J_{T^{\prime} S}(\Phi(u))\right)\right)+S(\Phi(u)) \tilde{T}^{\prime}(\Phi(u)) f(u, 0) \mathcal{L}^{N} .
\end{aligned}
$$

Lemma 3.17. Assume that $v \in\left(L^{1}\left(\mathbb{R}^{N}\right) \cap L^{\infty}\left(\mathbb{R}^{N}\right)\right)^{+}$. Let $(S, T) \in$ $\mathcal{T S U B} \cup \mathcal{T S U P \mathcal { R }}$. Then $a(u, \nabla \Phi(u)) \cdot \nabla\left(J_{T^{\prime} S}(\Phi(u))\right)$ is the absolutely continuous part of $\left(a(u, \nabla \Phi(u)), D\left(J_{T^{\prime} S}(\Phi(u))\right)\right)$ with respect to $\mathcal{L}^{N}$.

Lemma 3.17 is just a consequence of the fact that $\mathbf{a}(u, \nabla \Phi(u)) \in$ $X_{1}\left(\mathbb{R}^{N}\right)$. Its absolutely continuous part is $\mathbf{a}(u, \nabla \Phi(u)) \cdot \nabla J_{T^{\prime} S}(\Phi(u))$ 
since $\left(\mathbf{a}(u, \nabla \Phi(u)), D J_{T^{\prime} S}(\Phi(u))\right)^{s}$ is absolutely continuous with respect to $D J_{T^{\prime} S}(\Phi(u))^{s}[\mathbf{1 0}]$.

Before proving Lemmas 3.15 and 3.16, let us give the proof of (3.36).

Proof of Lemma 3.14: Using (3.38), we have

$$
\begin{aligned}
& \left(\mathbf{a}(u, \nabla \Phi(u)), D\left(J_{T^{\prime} S}(\Phi(u))\right)\right) \\
& \quad=\left(\mathbf{a}(u, \nabla \Phi(u)), D\left(J_{T^{\prime} S}(\Phi(u))\right)\right)^{a c}+\left(\mathbf{a}(u, \nabla \Phi(u)), D\left(J_{T^{\prime} S}(\Phi(u))\right)\right)^{s} \\
& \quad \geq \mathbf{a}(u, \nabla \Phi(u)) \cdot \nabla\left(J_{T^{\prime} S}(\Phi(u))\right)+\left(f_{S: T}^{\Phi}\left(\Phi(u), D T_{a, b}(\Phi(u))\right)\right)^{s} \\
& \quad=\mathbf{a}(u, \nabla \Phi(u)) \cdot \nabla\left(J_{T^{\prime} S}(\Phi(u))\right)+\left(h_{S: T}^{\Phi}\left(\Phi(u), D T_{a, b}(\Phi(u))\right)\right)^{s} \\
& \quad=h_{S: T}^{\Phi}\left(\Phi(u), D T_{a, b}(\Phi(u))\right),
\end{aligned}
$$

and (3.36) holds.

Proof of Lemma 3.15: Let us consider first the case $(S, T) \in \mathcal{T} \mathcal{S} \mathcal{B}$, that is, $S, T \in \mathcal{P}, S \geq 0, S^{\prime} \geq 0$ and $T \geq 0, T^{\prime} \geq 0$, and $p(\bar{z})=\tilde{p}\left(T_{a, b}(\bar{z})\right)$ for some $0<a<b$, where $\tilde{p}$ is differentiable in a neighborhood of $[a, b]$ and $p$ represents either $S$ or $T$.

Let $0 \leq \phi \in C_{c}^{2}\left(\mathbb{R}^{N}\right)$. Observe that

$$
J_{T^{\prime} S}(\bar{z})=\int_{0}^{\bar{z}} T^{\prime}(r) S(r) d r \leq\|S\|_{\infty} \int_{0}^{\bar{z}} T^{\prime}(r) d r=\|S\|_{\infty} T(\bar{z}) .
$$

Then, multiplying $(3.22)$ by $J_{T^{\prime} S}\left(\Phi\left(u_{n}\right)\right) \phi$ and integrating by parts, we obtain

$$
\begin{aligned}
\int_{\mathbb{R}^{N}} \phi \mathbf{a} & \left(u_{n}, \nabla \Phi\left(u_{n}\right)\right) \cdot \nabla\left(J_{T^{\prime} S}\left(\Phi\left(u_{n}\right)\right)\right) d x \\
& +\frac{1}{n} \int_{\mathbb{R}^{N}} \phi \nabla u_{n} \cdot \nabla\left(J_{T^{\prime} S}\left(\Phi\left(u_{n}\right)\right)\right) d x \\
= & \int_{\mathbb{R}^{N}}\left(v-u_{n}\right) J_{T^{\prime} S}\left(\Phi\left(u_{n}\right)\right) \phi d x \\
& -\int_{\mathbb{R}^{N}} J_{T^{\prime} S}\left(\Phi\left(u_{n}\right)\right) \mathbf{a}\left(u_{n}, \nabla \Phi\left(u_{n}\right)\right) \cdot \nabla \phi d x \\
& -\frac{1}{n} \int_{\mathbb{R}^{N}} J_{T^{\prime} S}\left(\Phi\left(u_{n}\right)\right) \nabla u_{n} \cdot \nabla \phi d x .
\end{aligned}
$$

Since

$$
\begin{aligned}
\frac{1}{n} \int_{\mathbb{R}^{N}} J_{T^{\prime} S}\left(\Phi\left(u_{n}\right)\right) & \nabla u_{n} \cdot \nabla \phi d x \\
& =-\frac{1}{n} \int_{\mathbb{R}^{N}} J_{J_{T \circ \Phi^{\prime} S \circ \Phi}}\left(u_{n}\right) \cdot \Delta \phi d x \rightarrow 0 \quad \text { as } n \rightarrow \infty,
\end{aligned}
$$


letting $n \rightarrow \infty$ and taking into account (3.39) we get

$$
\begin{aligned}
& \limsup _{n} \int_{\mathbb{R}^{N}} \phi \mathbf{a}\left(u_{n}, \nabla \Phi\left(u_{n}\right)\right) \cdot \nabla\left(J_{T^{\prime} S}\left(\Phi\left(u_{n}\right)\right)\right) d x \\
& \leq \int_{\mathbb{R}^{N}}(v-u) J_{T^{\prime} S}(\Phi(u)) \phi d x \\
&-\int_{\mathbb{R}^{N}} J_{T^{\prime} S}(\Phi(u)) \mathbf{a}(u, \nabla \Phi(u)) \cdot \nabla \phi d x \\
&=-\int_{\mathbb{R}^{N}} \operatorname{div} \mathbf{a}(u, \nabla \Phi(u)) J_{T^{\prime} S}(\Phi(u)) \phi d x \\
&-\int_{\mathbb{R}^{N}} J_{T^{\prime} S}(\Phi(u)) \mathbf{a}(u, \nabla \Phi(u)) \cdot \nabla \phi \\
&= \int_{\mathbb{R}^{N}} \phi\left(\mathbf{a}(u, \nabla \Phi(u)), D\left(J_{T^{\prime} S}(\Phi(u))\right)\right) .
\end{aligned}
$$

To consider the case where $(S, T) \in \mathcal{T S U P \mathcal { R }}$ it suffices to observe that

$$
\begin{aligned}
0 \leq J_{T^{\prime} S}(\bar{z}) & =\int_{0}^{\bar{z}} T^{\prime}(r) S(r) d r \leq\|S\|_{\infty} \int_{0}^{\bar{z}}-T^{\prime}(r) d r \\
& =-\|S\|_{\infty} T(\bar{z})=\|S\|_{\infty}|T(\bar{z})|
\end{aligned}
$$

and proceed as in the case where $(S, T) \in \mathcal{T S U B}$.

Proof of Lemma 3.16: Let us consider the case $(S, T) \in \mathcal{T S U B}$, that is, $S, T \in \mathcal{P}, S \geq 0, S^{\prime} \geq 0$ and $T \geq 0, T^{\prime} \geq 0$, and $T(\bar{z})=\tilde{T}\left(T_{a, b}(\bar{z})\right)$ for some $0<a<b$, where $\tilde{T}$ is differentiable in a neighborhood of $[a, b]$.

As usual, we write $w=\Phi(u)$ and $w_{n}=\Phi\left(u_{n}\right)$. Using that $T\left(w_{n}\right)=$ $\tilde{T}\left(T_{a, b}\left(w_{n}\right)\right)$ we have

$$
\begin{aligned}
a\left(u_{n}, \nabla w_{n}\right) \cdot \nabla T\left(w_{n}\right) & =a\left(u_{n}, \nabla w_{n}\right) \cdot \nabla w_{n} \tilde{T}^{\prime}\left(T_{a, b}\left(w_{n}\right)\right) T_{a, b}^{\prime}\left(w_{n}\right) \\
& =a\left(u_{n}, \nabla w_{n}\right) \cdot \nabla T_{a, b}\left(w_{n}\right) \tilde{T}^{\prime}\left(T_{a, b}\left(w_{n}\right)\right) T_{a, b}^{\prime}\left(w_{n}\right) \\
& =a\left(u_{n}, \nabla w_{n}\right) \cdot \nabla T_{a, b}\left(w_{n}\right) \tilde{T}^{\prime}\left(w_{n}\right) .
\end{aligned}
$$

Using the convexity of $f^{\Phi}(\bar{z}, \xi)$ in $\xi$ we have

$$
\left.f^{\Phi}\left(w_{n}, \nabla T_{a, b}\left(w_{n}\right)\right) \leq \mathbf{a}^{\Phi}\left(w_{n}, \nabla T_{a, b}\left(w_{n}\right)\right)\right) \cdot \nabla T_{a, b}\left(w_{n}\right)+f^{\Phi}\left(w_{n}, 0\right)
$$

and using

$$
\left.\mathbf{a}^{\Phi}\left(w_{n}, \nabla T_{a, b}\left(w_{n}\right)\right)\right) \cdot \nabla T_{a, b}\left(w_{n}\right)=\mathbf{a}\left(u_{n}, \nabla w_{n}\right) \cdot \nabla T_{a, b}\left(w_{n}\right),
$$


(true since $T_{a, b}^{\prime}=0$ or 1 a.e.) and the fact that $S \tilde{T}^{\prime} \geq 0$, we have

$$
\begin{aligned}
\int_{\mathbb{R}^{N}} \phi & S\left(w_{n}\right) \tilde{T}^{\prime}\left(w_{n}\right) f^{\Phi}\left(w_{n}, \nabla T_{a, b}\left(w_{n}\right)\right) d x \\
\leq & \int_{\mathbb{R}^{N}} \phi S\left(w_{n}\right) \tilde{T}^{\prime}\left(w_{n}\right) \mathbf{a}\left(u_{n}, \nabla w_{n}\right) \cdot \nabla T_{a, b}\left(w_{n}\right) d x \\
& +\int_{\mathbb{R}^{N}} \phi S\left(w_{n}\right) \tilde{T}^{\prime}\left(w_{n}\right) f^{\Phi}\left(w_{n}, 0\right) d x \\
= & \int_{\mathbb{R}^{N}} \phi \mathbf{a}\left(u_{n}, \nabla w_{n}\right) \cdot \nabla\left(J_{T^{\prime} S}\left(w_{n}\right)\right) d x \\
& +\int_{\mathbb{R}^{N}} \phi S\left(w_{n}\right) \tilde{T}^{\prime}\left(w_{n}\right) f^{\Phi}\left(w_{n}, 0\right) d x
\end{aligned}
$$

(Notice that $\tilde{T}^{\prime}\left(w_{n}\right) \nabla T_{a, b}\left(w_{n}\right)=\nabla T\left(w_{n}\right)$.)

Then, since $\mathcal{R}\left(\phi S \tilde{T}^{\prime} f^{\Phi}, T_{a, b}\right)$ is lower semi-continuous respect to the $L^{1}$-convergence and (3.37), letting $n \rightarrow \infty$ we obtain

$$
\begin{aligned}
\left\langle f_{S: \tilde{T}^{\Phi}}^{\Phi}\left(w, D T_{a, b}(w)\right), \phi\right\rangle= & \mathcal{R}\left(\phi S \tilde{T}^{\prime} f^{\Phi}, T_{a, b}\right)(w) \\
\leq & \liminf _{n} \mathcal{R}\left(\phi S \tilde{T}^{\prime} f^{\Phi}, T_{a, b}\right)\left(w_{n}\right) d x \\
= & \liminf _{n} \int_{\mathbb{R}^{N}} \phi S\left(w_{n}\right) \tilde{T}^{\prime}\left(w_{n}\right) f^{\Phi}\left(w_{n}, \nabla T_{a, b}\left(w_{n}\right)\right) d x \\
\leq & \liminf _{n} \int_{\mathbb{R}^{N}} \phi \mathbf{a}\left(u_{n}, \nabla w_{n}\right) \cdot \nabla\left(J_{T^{\prime} S}\left(w_{n}\right)\right) d x \\
& +\int_{\mathbb{R}^{N}} \phi S\left(w_{n}\right) \tilde{T}^{\prime}\left(w_{n}\right) f^{\Phi}\left(w_{n}, 0\right) d x \\
\leq & \lim _{n} \sup \int_{\mathbb{R}^{N}} \phi \mathbf{a}\left(u_{n}, \nabla w_{n}\right) \cdot \nabla\left(J_{T^{\prime} S}\left(w_{n}\right)\right) d x \\
& +\int_{\mathbb{R}^{N}} \phi S(w) \tilde{T}^{\prime}(w) f^{\Phi}(w, 0) d x \\
\leq & \int_{\mathbb{R}^{N}} \phi\left(\mathbf{a}(u, \nabla w), D\left(J_{T^{\prime} S}(w)\right)\right) \\
& +\int_{\mathbb{R}^{N}} \phi S(w) \tilde{T}^{\prime}(w) f(u, 0) d x
\end{aligned}
$$

and (3.38) holds since $f_{S: \tilde{T}}^{\Phi}\left(w, D T_{a, b}(w)\right)=f_{S: T}^{\Phi}\left(w, D T_{a, b}(w)\right)$.

The proof in the case where $(S, T) \in \mathcal{T} \mathcal{S U} \mathcal{P} \mathcal{E} \mathcal{R}$ is identical to the case where $(S, T) \in \mathcal{T} \mathcal{S U B}$. 


\subsubsection{The proof of uniqueness of entropy solutions for the el- liptic problem.}

Step 1. Preparation. Given $v, \bar{v} \in L^{1}\left(\mathbb{R}^{N}\right) \cap L^{\infty}\left(\mathbb{R}^{N}\right), v \geq 0, \bar{v} \geq 0$, let $u, \bar{u} \in L^{1}\left(\mathbb{R}^{N}\right) \cap L^{\infty}\left(\mathbb{R}^{N}\right), u, \bar{u} \geq 0$, be two entropy solutions of the problems

$$
u-\operatorname{div} \mathbf{a}(u, D \Phi(u))=v \quad \text { in } \quad \mathbb{R}^{N},
$$

and

$$
\bar{u}-\operatorname{div} \mathbf{a}(\bar{u}, D \Phi(\bar{u}))=\bar{v} \quad \text { in } \quad \mathbb{R}^{N},
$$

respectively. Recall that $\mathbf{a}(u, D \Phi(u)), \mathbf{a}(\bar{u}, D \Phi(\bar{u})) \in L^{1}\left(\mathbb{R}^{N}\right) \cap L^{\infty}\left(\mathbb{R}^{N}\right)$.

To simplify, let us write $w(y)=\Phi(u(y)), \bar{w}(x)=\Phi(\bar{u}(x))$. Let $\rho_{n}$ be a classical sequence of mollifiers in $\mathbb{R}^{N}, b>a>2 \varepsilon>0$. Let us write

$$
\xi_{n}(x, y)=\rho_{n}(x-y) \quad \text { and } \quad T=T_{a, b}^{a} .
$$

We need to consider truncature functions of the form

$$
S_{\varepsilon, l}(r):=T_{\varepsilon}(r-l)^{+}=T_{l, l+\varepsilon}(r)-l \in \mathcal{P}^{+}
$$

and

$$
S_{\varepsilon}^{l}(r):=T_{\varepsilon}(r-l)^{-}+\varepsilon=T_{l-\varepsilon, l}(r)+\varepsilon-l \in \mathcal{P}^{+},
$$

where $l \geq 0$. Observe that

$$
S_{\varepsilon}^{l}(r)=-T_{\varepsilon}(l-r)^{+}+\varepsilon .
$$

If we denote $\mathbf{z}(y)=\mathbf{a}(u(y), \nabla w(y))$ and $\overline{\mathbf{z}}(x)=\mathbf{a}(\bar{u}(x), \nabla \bar{w}(x))$, we have

$$
u-\operatorname{div}(\mathbf{z})=v \quad \text { and } \quad \bar{u}-\operatorname{div}(\overline{\mathbf{z}})=\bar{v} \quad \text { in } \quad \mathcal{D}^{\prime}\left(\mathbb{R}^{N}\right) .
$$

Then, multiplying the first equation by $T(w(y)) S_{\varepsilon, \bar{w}(x)}(w(y)) \xi_{n}(x, y)$, the second by $T(\bar{w}(x)) S_{\varepsilon}^{w(y)}(\bar{w}(x)) \xi_{n}(x, y)$ and integrating by parts, we obtain

$$
\begin{aligned}
\int_{\mathbb{R}^{N}} u(y) & T(w(y)) T_{\varepsilon}(w(y)-\bar{w}(x))^{+} \xi_{n}(x, y) d y \\
& +\int_{\mathbb{R}^{N}} \xi_{n}(x, y)\left(\mathbf{z} \cdot D_{y}\left(T(w) S_{\varepsilon, \bar{w}(x)}(w(y))\right)\right) \\
& +\int_{\mathbb{R}^{N}} T(w(y)) S_{\varepsilon, \bar{w}(x)}(w(y)) \mathbf{z}(y) \cdot \nabla_{y} \xi_{n}(x, y) d y \\
= & \int_{\mathbb{R}^{N}} v(y) T(w(y)) T_{\varepsilon}(w(y)-\bar{w}(x))^{+} \xi_{n}(x, y) d y
\end{aligned}
$$


and

$$
\begin{aligned}
& -\int_{\mathbb{R}^{N}} \bar{u}(x) T(\bar{w}(x))\left(T_{\varepsilon}(w(y)-\bar{w}(x))^{+}-\varepsilon\right) \xi_{n}(x, y) d x \\
& +\int_{\mathbb{R}^{N}} \xi_{n}(x, y)\left(\overline{\mathbf{z}} \cdot D_{x}\left(T(\bar{w}) S_{\varepsilon}^{w(y)}(\bar{w})\right)\right) \\
& +\int_{\mathbb{R}^{N}} T(\bar{w}) S_{\varepsilon}^{w(y)}(\bar{w}(x)) \overline{\mathbf{z}}(x) \cdot \nabla_{x} \xi_{n}(x, y) d x \\
& =-\int_{\mathbb{R}^{N}} \bar{v}(x) T(\bar{w}(x))\left(T_{\varepsilon}(w(y)-\bar{w}(x))^{+}-\varepsilon\right) \xi_{n}(x, y) d x .
\end{aligned}
$$

Notice that, by Lemma 3.14, the couplings $\left(\mathbf{z} \cdot D_{y}\left(T(w) S_{\varepsilon, \bar{w}(x)}(w(y))\right)\right)$ and $\left(\overline{\mathbf{z}} \cdot D_{x}\left(T(\bar{w}) S_{\varepsilon}^{w(y)}(\bar{w})\right)\right)$ are Radon measures.

Integrating (3.43) in $x$ and (3.44) in $y$, and adding both identities we obtain

$$
\begin{aligned}
\int_{\mathbb{R}^{N}} \int_{\mathbb{R}^{N}}(u(y) T(w(y))-\bar{u}(x) T(\bar{w}(x))) T_{\varepsilon}(w(y)-\bar{w}(x))^{+} \xi_{n}(x, y) d x d y \\
\quad+\varepsilon \int_{\mathbb{R}^{N}} \int_{\mathbb{R}^{N}}(\bar{u}(x)-\bar{v}(x)) T(\bar{w}(x)) \xi_{n}(x, y) d x d y \\
\quad+\int_{\mathbb{R}^{N}}\left(\int_{\mathbb{R}^{N}} \xi_{n}(x, y)\left(\mathbf{z} \cdot D_{y}\left(T(w) S_{\varepsilon, \bar{w}(x)}(w)\right)\right) d x\right. \\
+\int_{\mathbb{R}^{N} \times \mathbb{R}^{N}} T(w(y)) S_{\varepsilon, \bar{w}(x)}(w(y)) \mathbf{z}(y) \cdot \nabla_{y} \xi_{n}(x, y) d y d x \\
\quad+\int_{\mathbb{R}^{N}}\left(\int_{\mathbb{R}^{N}} \xi_{n}(x, y)\left(\overline{\mathbf{z}} \cdot D_{x}\left(T(\bar{w}) S_{\varepsilon}^{w(y)}(\bar{w})\right)\right)\right) d y \\
\quad+\int_{\mathbb{R}^{N} \times \mathbb{R}^{N}} T(\bar{w}(x)) S_{\varepsilon}^{w(y)}(\bar{w}(x)) \overline{\mathbf{z}}(x) \cdot \nabla_{x} \xi_{n}(x, y) d x d y \\
=\int_{\mathbb{R}^{N}} \int_{\mathbb{R}^{N}}(v(y) T(w(y))-\bar{v}(x) T(\bar{w}(x))) T_{\varepsilon}(w(y)-\bar{w}(x))^{+} \xi_{n}(x, y) d x d y .
\end{aligned}
$$

Let $I_{1}, I_{2}$ be, respectively, the first term and the rest of the terms at the left hand side of the above identity, and let $I_{3}$ be the right hand side term. From now on, since $u, \mathbf{z}$ are always functions of $y$, and $\bar{u}, \overline{\mathbf{z}}$ are always functions of $x$, to make our expressions shorter, we shall omit the 
arguments except on sub- and superindices and in some additional cases where we find useful to remind them.

Step 2. The estimate on $I_{2}$. Let us prove that

$$
\frac{1}{\varepsilon} I_{2} \geq\left\|\xi_{n}\right\|_{\infty} O(\varepsilon)+\int_{\mathbb{R}^{N}} \int_{\mathbb{R}^{N}} \operatorname{div}(\overline{\mathbf{z}}) T(\bar{w}) \xi_{n} d x d y,
$$

where $o(\varepsilon) \rightarrow 0$ as $\varepsilon \rightarrow 0$. Since $\bar{u}-\bar{v}=\operatorname{div} \overline{\mathbf{z}}$ and $\nabla_{y} \xi_{n}(x, y)+$ $\nabla_{x} \xi_{n}(x, y)=0$, we have

$$
\begin{aligned}
I_{2}= & \varepsilon \int_{\mathbb{R}^{N}} \int_{\mathbb{R}^{N}} \operatorname{div}(\overline{\mathbf{z}}) T(\bar{w}) \xi_{n} d x d y+\int_{\mathbb{R}^{N}}\left(\int_{\mathbb{R}^{N}} \xi_{n}\left(\mathbf{z} \cdot D_{y}\left(T(w) S_{\varepsilon, \bar{w}(x)}(w)\right)\right)\right) d x \\
& -\int_{\mathbb{R}^{N} \times \mathbb{R}^{N}} T(\bar{w}) S_{\varepsilon}^{w(y)}(\bar{w}) \overline{\mathbf{z}} \cdot \nabla_{y} \xi_{n} d x d y+\int_{\mathbb{R}^{N}}\left(\int_{\mathbb{R}^{N}} \xi_{n}\left(\overline{\mathbf{z}} \cdot D_{x}\left(T(\bar{w}) S_{\varepsilon}^{w(y)}(\bar{w})\right)\right)\right) d y \\
& \left.-\int_{\mathbb{R}^{N} \times \mathbb{R}^{N}} T(w) S_{\varepsilon, \bar{w}(x)}(w)\right) \mathbf{z} \cdot \nabla_{x} \xi_{n} d y d x \\
= & \varepsilon \int_{\mathbb{R}^{N}} \int_{\mathbb{R}^{N}} \operatorname{div}(\overline{\mathbf{z}}) T(\bar{w}) \xi_{n} d x d y+\int_{\mathbb{R}^{N}}\left(\int_{\mathbb{R}^{N}} \xi_{n}\left(\mathbf{z} \cdot D_{y}\left(T(w) S_{\varepsilon, \bar{w}(x)}(w)\right)\right)\right) d x \\
& +\int_{\mathbb{R}^{N} \times \mathbb{R}^{N}} \xi_{n} \overline{\mathbf{z}} \cdot D_{y}\left(T(\bar{w}) T_{\varepsilon}(\bar{w}-w)^{-}\right) d x d y+\int_{\mathbb{R}^{N}}\left(\int_{\mathbb{R}^{N}} \xi_{n}\left(\overline{\mathbf{z}} \cdot D_{x}\left(T(\bar{w}) S_{\varepsilon}^{w(y)}(\bar{w})\right)\right)\right) d y \\
& \left.+\int_{\mathbb{R}^{N} \times \mathbb{R}^{N}} \xi_{n} \mathbf{z} \cdot D_{x}\left(T(w) T_{\varepsilon}(w-\bar{w})^{+}\right)\right) d y d x \\
= & \varepsilon \int_{\mathbb{R}^{N}} \int_{\mathbb{R}^{N}} \operatorname{div}(\overline{\mathbf{z}}) T(\bar{w}) \xi_{n} d x d y+\int_{\mathbb{R}^{N}}\left(\int_{\mathbb{R}^{N}} \xi_{n}\left(\mathbf{z} \cdot D_{y} J_{\left.T^{\prime} S_{\varepsilon, \bar{w}(x)}\right)}(w)\right)\right) d x \\
& +\int_{\mathbb{R}^{N}}\left(\int_{\mathbb{R}^{N}} \xi_{n}\left(\overline{\mathbf{z}} \cdot D_{x} J_{T^{\prime} S_{\varepsilon}^{w(y)}}(\bar{w})\right)\right) d y+\int_{\mathbb{R}^{N}}\left(\int_{\mathbb{R}^{N}} \xi_{n}\left(\mathbf{z}, D_{y} J_{T S_{\varepsilon, \bar{w}}^{\prime}(x)}(w)\right)\right) d x \\
& -\int_{\mathbb{R}^{N}} T(\bar{w})\left(\int_{\mathbb{R}^{N}} \xi_{n} \overline{\mathbf{z}} \cdot D_{y} T_{\varepsilon}(w-\bar{w})^{+}\right) d x+\int_{\mathbb{R}^{N}}\left(\int_{\mathbb{R}^{N}} \xi_{n}\left(\overline{\mathbf{z}} \cdot D_{x} J_{T S_{\varepsilon}^{w(y)}}(\bar{w})\right)\right) d y \\
& +\int_{\mathbb{R}^{N}} T(w(y))\left(\int_{\mathbb{R}^{N}} \xi_{n} \mathbf{z} \cdot D_{x} T_{\varepsilon}(w-\bar{w})^{+}\right) d y \\
= & I_{2,1}+I_{2,2},
\end{aligned}
$$

where $I_{2,1}$ denotes the sum of the first three terms and $I_{2,2}$ denotes the sum from the fourth to the seventh terms.

Let us consider the second and third terms in $I_{2,1}$. Since

$$
h_{S_{\varepsilon, \bar{w}(x)}^{\Phi}}^{\Phi}\left(w, D_{y} T(w)\right) \leq\left(\mathbf{z} \cdot D_{y} J_{T^{\prime} S_{\varepsilon, \bar{w}(x)}}(w)\right)
$$

and

$$
h_{S_{\varepsilon}^{w(y)}}^{\Phi}\left(\bar{w}, D_{x} T(\bar{w})\right) \leq\left(\overline{\mathbf{z}} \cdot D_{x} J_{T^{\prime} S_{\varepsilon}^{w(y)}}(\bar{w})\right)
$$

as measures in $\mathbb{R}^{N}$, we have

$$
\left.\int_{\mathbb{R}^{N}}\left(\int_{\mathbb{R}^{N}} \xi_{n}\left(\mathbf{z} \cdot D_{y} J_{T^{\prime} S_{\varepsilon, \bar{w}(x)}}(w)\right)\right)\right) d x \geq 0
$$


and

$$
\int_{\mathbb{R}^{N}}\left(\int_{\mathbb{R}^{N}} \xi_{n}\left(\overline{\mathbf{z}} \cdot D_{x} J_{T^{\prime} S_{\varepsilon}^{w(y)}}(\bar{w})\right)\right) d y \geq 0 .
$$

Hence,

$$
I_{2,1} \geq \varepsilon \int_{\mathbb{R}^{N}} \int_{\mathbb{R}^{N}} \operatorname{div}(\overline{\mathbf{z}}) T(\bar{w}) \xi_{n} d x d y .
$$

Let us write the term

$$
I_{2,2}=I_{2,2}(a c)+I_{2,2}(s)
$$

where $I_{2,2}(a c)$ contains the absolutely continuous parts of $I_{2,2}$ while $I_{2,2}(s)$ contains its singular parts. Now,

$$
\begin{aligned}
I_{2,2}(a c)= & \int_{\mathbb{R}^{N}} \int_{\mathbb{R}^{N}} \xi_{n} T(w) \mathbf{z} \cdot \nabla_{y} T_{\varepsilon}(w-\bar{w})^{+} d y d x \\
& -\int_{\mathbb{R}^{N}} \int_{\mathbb{R}^{N}} \xi_{n} T(\bar{w}) \overline{\mathbf{z}} \cdot \nabla_{y} T_{\varepsilon}(w-\bar{w})^{+} d y d x \\
& -\int_{\mathbb{R}^{N}} \int_{\mathbb{R}^{N}} \xi_{n} T(\bar{w}) \overline{\mathbf{z}} \cdot \nabla_{x} T_{\varepsilon}(w-\bar{w})^{+} d x d y \\
& +\int_{\mathbb{R}^{N}} \int_{\mathbb{R}^{N}} \xi_{n} T(w) \mathbf{z} \cdot \nabla_{x} T_{\varepsilon}(w-\bar{w})^{+} d x d y \\
= & \int_{\mathbb{R}^{N}} \int_{\mathbb{R}^{N}} \xi_{n}(\mathbf{z} T(w)-\overline{\mathbf{z}} T(\bar{w}))\left(\nabla_{y} T_{\varepsilon}(w-\bar{w})^{+}+\nabla_{x} T_{\varepsilon}(w-\bar{w})^{+}\right) d x d y \\
= & \int_{\mathbb{R}^{N}} \int_{\mathbb{R}^{N}} \xi_{n}(\mathbf{z}-\overline{\mathbf{z}}) T(w)\left(\nabla_{y} T_{\varepsilon}(w-\bar{w})^{+}+\nabla_{x} T_{\varepsilon}(w-\bar{w})^{+}\right) d x d y \\
& +\int_{\mathbb{R}^{N}} \int_{\mathbb{R}^{N}} \xi_{n} \overline{\mathbf{z}}(T(w)-T(\bar{w}))\left(\nabla_{y} T_{\varepsilon}(w-\bar{w})^{+}+\nabla_{x} T_{\varepsilon}(w-\bar{w})^{+}\right) d x d y \\
= & : T_{1}\left(I_{2,2}(a c)\right)+T_{2}\left(I_{2,2}(a c)\right) .
\end{aligned}
$$

Notice that all integrals are well defined because of the truncatures. Let us estimate $T_{1}\left(I_{2,2}(a c)\right)$.

Bound on $T_{1}\left(I_{2,2}(a c)\right)$. First, observe that

$$
\begin{aligned}
\nabla_{y} T_{\varepsilon}(w-\bar{w}(x))^{+}(y) & =\chi_{[\bar{w}(x), \bar{w}(x)+\varepsilon]}(w(y)) \nabla_{y} w(y) \\
\nabla_{x} T_{\varepsilon}(w(y)-\bar{w})^{+}(x) & =-\chi_{[w(y)-\varepsilon, w(y)]}(\bar{w}(x)) \nabla_{x} \bar{w}(x) \\
& =-\chi_{[\bar{w}(x), \bar{w}(x)+\varepsilon]}(w(y)) \nabla_{x} \bar{w}(x) .
\end{aligned}
$$


By (3.10), we have

$$
\begin{aligned}
& T_{1}\left(I_{2,2}(a c)\right)=\int_{\mathbb{R}^{N}} \int_{\mathbb{R}^{N}} \xi_{n}(\mathbf{z}-\overline{\mathbf{z}}) T(w)\left(\nabla_{y} w-\nabla_{x} \bar{w}\right) \chi_{[\bar{w}(x), \bar{w}(x)+\varepsilon]}(w) d x d y \\
& \geq-C \int_{\mathbb{R}^{N}} \int_{\mathbb{R}^{N}} T(w) \chi_{\{w \geq a\}} \xi_{n} \chi_{[\bar{w}(x), \bar{w}(x)+\varepsilon]}(w)\left|u^{\bar{m}}-\bar{u}^{\bar{m}}\right|\left\|\nabla_{y} w-\nabla_{x} \bar{w}\right\| d x d y .
\end{aligned}
$$

Let us decompose the previous integral into:

$$
\begin{aligned}
& T_{1,1}\left(I_{2,2}(a c)\right) \\
& :=\int_{\mathbb{R}^{N}} \int_{\mathbb{R}^{N}} T(w) \chi_{\{w \geq a\}} \xi_{n} \chi_{[\bar{w}(x), \bar{w}(x)+\varepsilon]}(w)\left|u^{\bar{m}}-\bar{u}^{\bar{m}}\right|\left\|\nabla_{y} w\right\| d x d y,
\end{aligned}
$$

and

$$
\begin{aligned}
& T_{1,2}\left(I_{2,2}(a c)\right) \\
& :=\int_{\mathbb{R}^{N}} \int_{\mathbb{R}^{N}} T(w) \chi_{\{w \geq a\}} \xi_{n} \chi_{[\bar{w}(x), \bar{w}(x)+\varepsilon]}(w)\left|u^{\bar{m}}-\bar{u}^{\bar{m}}\right|\left\|\nabla_{x} \bar{w}\right\| d x d y .
\end{aligned}
$$

Bound on $T_{1,1}\left(I_{2,2}(a c)\right)$. Let us write $z^{\bar{m}}=\left(\Phi^{-1}(\Phi(z))^{\bar{m}}\right.$. Let $\Psi(\bar{z})=$ $\left(\Phi^{-1}(\bar{z})\right)^{\bar{m}}$. Then $\Psi \in W^{1, \infty}([a, b])$ for all $0<a<b$. Observe that $a \leq w \leq \Phi\left(\|u\|_{\infty}\right), \frac{a}{2} \leq \bar{w} \leq \Phi\left(\|\bar{u}\|_{\infty}\right)$. Then

$$
\left|u^{\bar{m}}-\bar{u}^{\bar{m}}\right|=|\Psi(w)-\Psi(\bar{w})|=\Psi^{\prime}(s)|w-\bar{w}|
$$

for some intermediate value $s$ in $[\min (w, \bar{w}), \max (w, \bar{w})]$. Thus

$$
\left|u^{\bar{m}}-\bar{u}^{\bar{m}}\right| \leq C(a)|w-\bar{w}|,
$$

where we explicitly write the dependence of the constant $C(a)$ on $a$. We have

$$
\begin{aligned}
T_{1,1}\left(I_{2,2}(a c)\right) & \leq \varepsilon C(a) \int_{\mathbb{R}^{N}} \int_{\mathbb{R}^{N}} T(w) \chi_{\{w \geq a\}} \xi_{n} \chi_{[\bar{w}(x), \bar{w}(x)+\varepsilon]}(w)\left\|\nabla_{y} w\right\| d x d y \\
& \leq \varepsilon C(a) \int_{\mathbb{R}^{N}} T(\bar{w}+\varepsilon) \int_{\mathbb{R}^{N}} \chi_{\{w \geq a\}} \xi_{n} \chi_{[\bar{w}(x), \bar{w}(x)+\varepsilon]}(w)\left\|\nabla_{y} w\right\| d x d y
\end{aligned}
$$

Observe that

$$
\begin{aligned}
& \int_{\mathbb{R}^{N}} \chi_{\{w \geq a\}} \chi_{[\bar{w}(x), \bar{w}(x)+\varepsilon]}(w)\left\|\nabla_{y} w\right\| d y \\
&=\chi_{\{\bar{w} \geq a-\varepsilon\}} \int_{\bar{w}(x)}^{\bar{w}(x)+\varepsilon} P(\{w \geq \lambda\}) d \lambda d x \leq o(\varepsilon) \chi_{\{\bar{w} \geq a-\varepsilon\}}
\end{aligned}
$$


with $o(\varepsilon) \rightarrow 0$ as $\varepsilon \rightarrow 0$ since $T_{a, \infty}(w)-a \in B V\left(\mathbb{R}^{N}\right)$. Then

$$
\begin{aligned}
T_{1,1}\left(I_{2,2}(a c)\right) & \leq \varepsilon O(\varepsilon) C(a)\left\|\xi_{n}\right\|_{\infty} \int_{\mathbb{R}^{N}} \chi_{\{\bar{w} \geq a-\varepsilon\}} T(\bar{w}+\varepsilon) d x \\
& \leq \varepsilon O(\varepsilon) C(a)\left\|\xi_{n}\right\|_{\infty} \int_{\mathbb{R}^{N}} \chi_{\{\bar{w} \geq a-\varepsilon\}} T(2 \bar{w}) \\
& \leq 2 \varepsilon O(\varepsilon) C(a)\left\|\xi_{n}\right\|_{\infty} \int_{\mathbb{R}^{N}} \chi_{\{\bar{w} \geq a-\varepsilon\}} \bar{w},
\end{aligned}
$$

since $a>2 \varepsilon$. Observe that since $a-\varepsilon \geq a-\frac{a}{2}=\frac{a}{2}$ we have

$$
\int_{\mathbb{R}^{N}} \chi_{\{\bar{w} \geq a-\varepsilon\}} \bar{w} \leq \int_{\mathbb{R}^{N}} \chi_{\left\{\bar{w} \geq \frac{a}{2}\right\}} \bar{w}
$$

which is finite since $\bar{u} \in L^{1}\left(\mathbb{R}^{N}\right)^{+} \cap L^{\infty}\left(\mathbb{R}^{N}\right)^{+}$and $\left\{\bar{w} \geq \frac{a}{2}\right\}=\{\bar{u} \geq$ $\left.\Phi^{-1}\left(\frac{a}{2}\right)\right\}$ is a set of finite measure.

Bound on $T_{1,2}\left(I_{2,2}(a c)\right)$. Recall that

$$
\begin{aligned}
& T_{1,2}\left(I_{2,2}(a c)\right) \\
& :=\int_{\mathbb{R}^{N}} \int_{\mathbb{R}^{N}} T(w) \chi_{\{w \geq a\}} \xi_{n} \chi_{[\bar{w}(x), \bar{w}(x)+\varepsilon]}(w)\left|u^{\bar{m}}-\bar{u}^{\bar{m}}\right|\left\|\nabla_{x} \bar{w}\right\| d x d y .
\end{aligned}
$$

Then

$$
\begin{aligned}
& T_{1,2}\left(I_{2,2}(a c)\right) \\
& \quad \leq \varepsilon C(a) \int_{\mathbb{R}^{N}} \int_{\mathbb{R}^{N}} T(w) \chi_{\{w \geq a\}} \xi_{n} \chi_{[\Phi(u(x))-\varepsilon, \Phi(u(x))]}(\bar{w})\left\|\nabla_{x} \bar{w}\right\| d x d y \\
& \quad \leq \varepsilon C(a) \int_{\mathbb{R}^{N}} \chi_{\{w \geq a\}} T(w) \int_{\mathbb{R}^{N}} \xi_{n} \chi_{\{\bar{w} \geq a-\varepsilon\}} \chi_{[\Phi(u(x))-\varepsilon, \Phi(u(x))]}(\bar{w})\left\|\nabla_{x} \bar{w}\right\| d x d y .
\end{aligned}
$$

Observe that

$$
\begin{aligned}
\chi_{\{w \geq a\}} \int_{\mathbb{R}^{N}} \chi_{\{\bar{w} \geq a-\varepsilon\}} & \chi_{[\Phi(u(x))-\varepsilon, \Phi(u(x))]}(\bar{w})\left\|\nabla_{x} \bar{w}\right\| d y \\
& \leq \chi_{\{w \geq a\}} \int_{\Phi(u(x))-\varepsilon}^{\Phi(u(x))} P(\{\bar{w} \geq \lambda\}) d \lambda d x \leq o(\varepsilon)
\end{aligned}
$$

with $o(\varepsilon) \rightarrow 0$ as $\varepsilon \rightarrow 0$ since $T_{a / 2, \infty}(\bar{w})-\frac{a}{2} \in B V\left(\mathbb{R}^{N}\right)$. Then

$$
\begin{aligned}
T_{1,2}\left(I_{2,2}(a c)\right) & \leq \varepsilon o(\varepsilon) C(a)\left\|\xi_{n}\right\|_{\infty} \int_{\mathbb{R}^{N}} \chi_{\{w \geq a\}} T(w) d y \\
& \leq \varepsilon o(\varepsilon) C(a)\left\|\xi_{n}\right\|_{\infty} \int_{\mathbb{R}^{N}} \chi_{\{w \geq a\}} w
\end{aligned}
$$


Hence

$$
T_{1}\left(I_{2,2}(a c)\right) \geq-\varepsilon o(\varepsilon) C(a)\left\|\xi_{n}\right\|_{\infty}\left(\int_{\mathbb{R}^{N}} \chi_{\{w \geq a\}} w+2 \int_{\mathbb{R}^{N}} \chi_{\left\{\bar{w} \geq \frac{a}{2}\right\}} \bar{w}\right) .
$$

Since $u, \bar{u} \in L^{1}\left(\mathbb{R}^{N}\right) \cap L^{\infty}\left(\mathbb{R}^{N}\right)$, the last two integrals are bounded.

Bound on $T_{2}\left(I_{2,2}(a c)\right)$. Similarly, since $T$ has Lipschitz constant equal to 1 , we have

$$
\begin{aligned}
& \left|T_{2}\left(I_{2,2}(a c)\right)\right| \\
& =\left|\int_{\mathbb{R}^{N}} \int_{\mathbb{R}^{N}} \xi_{n} \overline{\mathbf{z}}(T(w)-T(\bar{w}))\left(\nabla_{y} w-\nabla_{x} \bar{w}\right) \chi_{[\bar{w}(x), \bar{w}(x)+\varepsilon]}(w) d x d y\right| \\
& \leq M \int_{\mathbb{R}^{N}} \int_{\mathbb{R}^{N}} \chi_{\{w \geq a-\varepsilon\}} \chi_{\{\bar{w} \geq a-\varepsilon\}} \chi_{\{0 \leq w-\bar{w} \leq \varepsilon\}} \xi_{n} \bar{u}^{\bar{m}}|w-\bar{w}|\left\|\nabla_{y} w-\nabla_{x} \bar{w}\right\| d x d y \\
& \leq M \varepsilon \int_{\mathbb{R}^{N}} \int_{\mathbb{R}^{N}} \chi_{\{w \geq a-\varepsilon\}} \chi_{\{\bar{w} \geq a-\varepsilon\}} \xi_{n} \chi_{\{0 \leq w-\bar{w} \leq \varepsilon\}} \bar{u}^{\bar{m}}\left\|\nabla_{y} w-\nabla_{x} \bar{w}\right\| d x d y \\
& \leq M \varepsilon \int_{\mathbb{R}^{N}} \int_{\mathbb{R}^{N}} \chi_{\{w \geq a-\varepsilon\}} \chi_{\{\bar{w} \geq a-\varepsilon\}} \xi_{n} \chi_{\{0 \leq w-\bar{w} \leq \varepsilon\}} \bar{u}^{\bar{m}}\left(\left\|\nabla_{y} w\right\|+\left\|\nabla_{x} \bar{w}\right\|\right) d x d y .
\end{aligned}
$$

As above we can bound

$$
\frac{1}{\varepsilon}\left|T_{2}\left(I_{2,2}(a c)\right)\right| \leq o(\varepsilon) .
$$

Hence,

$$
\frac{1}{\varepsilon} I_{2,2}(a c) \geq o(\varepsilon)
$$

Bound on $I_{2,2}(s)$. Finally, let us compute $I_{2,2}(s)$.

$$
\begin{aligned}
I_{2,2}(s)= & \int_{\mathbb{R}^{N}}\left(\int_{\mathbb{R}^{N}} \xi_{n} \mathbf{z} \cdot D_{y}^{s} J_{T S_{\varepsilon, \bar{w}(x)}^{\prime}}(w)\right) d x \\
& -\int_{\mathbb{R}^{N}}\left(\int_{\mathbb{R}^{N}} \xi_{n} T(\bar{w}) \overline{\mathbf{z}} \cdot D_{y}^{s} T_{\varepsilon}(w-\bar{w})^{+}\right) d x \\
& +\int_{\mathbb{R}^{N}}\left(\int_{\mathbb{R}^{N}} \xi_{n} \overline{\mathbf{z}} \cdot D_{x}^{s} J_{T S_{\varepsilon}^{w(y)^{\prime}}}(\bar{w})\right) d y \\
& +\int_{\mathbb{R}^{N}}\left(\int_{\mathbb{R}^{N}} \xi_{n} T(w) \mathbf{z} \cdot D_{x}^{s} T_{\varepsilon}(w-\bar{w})^{+}\right) d y \\
= & : T_{1}\left(I_{2,2}(s)\right)+T_{2}\left(I_{2,2}(s)\right) .
\end{aligned}
$$

Bound on $T_{1}\left(I_{2,2}(s)\right)$. Note that, if $\bar{u}(x)>0$, we have

$$
\mathbf{z} \cdot D_{y}^{s} J_{T S_{\varepsilon, \bar{w}(x)}^{\prime}}(w) \geq h_{T}^{\Phi}\left(w, D_{y} T_{\varepsilon}(w-\bar{w}(x))^{+}\right)^{s}=h_{T}^{\Phi}\left(u_{\varepsilon}, D_{y} u_{\varepsilon}\right)^{s} \geq 0
$$


where $u_{\varepsilon}(x, y)=T_{\bar{w}(x), \bar{w}(x)+\varepsilon}(w(y))$, and by $\left(\mathrm{H}_{6}\right)$ and $\left(\mathrm{H}_{7}\right)$, we have

$$
\overline{\mathbf{z}}(x) \cdot D_{y}^{s} T_{\varepsilon}(w-\bar{w}(x))^{+} \leq \varphi(\bar{u}(x)) \psi^{0}\left(\overrightarrow{D_{y}^{s} u_{\varepsilon}}\right)\left|D_{y}^{s} u_{\varepsilon}\right| .
$$

Since the integrand of the first term is positive and the support of $T(\bar{w})$ is contained in $\{\bar{w} \geq a\}$, we have

$$
\begin{aligned}
T_{1}\left(I_{2,2}(s)\right) \geq & \int_{\{\bar{w} \geq a\}}\left(\int_{\mathbb{R}^{N}} \xi_{n} \mathbf{z} \cdot D_{y}^{s} J_{T S_{\varepsilon, \bar{w}(x)}^{\prime}}(w)\right) d x \\
& -\int_{\{\bar{w} \geq a\}}\left(\int_{\mathbb{R}^{N}} \xi_{n} T(\bar{w}) \overline{\mathbf{z}} \cdot D_{y}^{s} T_{\varepsilon}(w-\bar{w})\right) d x \\
\geq & \int_{\{\bar{w} \geq a\}}\left(\int_{\mathbb{R}^{N}} \xi_{n} h_{T}^{\Phi}\left(u_{\varepsilon}, D_{y} u_{\varepsilon}\right)^{s}\right) d x \\
& -\int_{\{\bar{w} \geq a\}}\left(\int_{\mathbb{R}^{N}} \xi_{n} T(\bar{w}) \varphi(\bar{u}) \psi^{0}\left(\overrightarrow{D_{y}^{s} u_{\varepsilon}}\right)\left|D_{y}^{s} u_{\varepsilon}\right|\right) d x \\
= & \int_{\{\bar{w} \geq a\}}\left(\int_{\mathbb{R}^{N}} \xi_{n} T\left(u_{\varepsilon}\right) \varphi^{\Phi}\left(u_{\varepsilon}\right) \psi^{0}\left(\overrightarrow{D_{y}^{s} u_{\varepsilon}}\right)\left|D_{y}^{c} u_{\varepsilon}\right|\right) d x \\
& -\int_{\{\bar{w} \geq a\}}\left(\int_{\mathbb{R}^{N}} \xi_{n} T(\bar{w}) \varphi(\bar{u}) \psi^{0}\left(\overrightarrow{D_{y}^{s} u_{\varepsilon}}\right)\left|D_{y}^{c} u_{\varepsilon}\right|\right) d x \\
& +\int_{\{\bar{w} \geq a\}}\left(\int_{J_{u_{\varepsilon}}} \xi_{n} \frac{1}{\left(u_{\varepsilon}\right)^{+}(y)-\left(u_{\varepsilon}\right)^{-}(y)}\right. \\
& \left.\times\left(\int_{\left(u_{\varepsilon}\right)^{-}(y)}^{\left(u_{\varepsilon}\right)^{+}(y)} T(s) \varphi^{\Phi}(s) d s\right) \psi^{0}\left(\overrightarrow{D_{y}^{s} u_{\varepsilon}}\right)\left|D_{y}^{j} u_{\varepsilon}\right|\right) d x \\
& -\int_{\{\bar{w} \geq a\}}\left(\int_{\mathbb{R}^{N}} \xi_{n} T(\bar{w}) \varphi(\bar{u}) \psi^{0}\left(\overrightarrow{D_{y}^{s} u_{\varepsilon}}\right)\left|D_{y}^{j} u_{\varepsilon}\right|\right) d x \\
= & T_{1,1}\left(I_{2,2}(s)\right)+T_{1,2}\left(I_{2,2}(s)\right), \\
& \\
&
\end{aligned}
$$

where $T_{1,1}\left(I_{2,2}(s)\right)$ denotes the sum of the first and second terms of the above expression, and $T_{1,2}\left(I_{2,2}(s)\right)$ the sum of the third and fourth terms. Bound on $T_{1,1}\left(I_{2,2}(s)\right)$. Since $\Phi^{-1} \in W^{1, \infty}([a, b])$ for all $0<a<b$, we have that $T(\bar{z}) \varphi^{\Phi}(\bar{z})$ is Lipschitz on any interval $[a, b]$ with $a>0$. Using 
$\left(\mathrm{H}_{7}\right)$ we have

$$
\begin{aligned}
& \left|T_{1,1}\left(I_{2,2}(s)\right)\right| \\
& \quad \leq \int_{\mathbb{R}^{N}}\left(\int_{\{\bar{w} \geq a\}} \xi_{n}\left|T\left(u_{\varepsilon}\right) \varphi^{\Phi}\left(u_{\varepsilon}\right)-T(\bar{w}) \varphi^{\Phi}(\bar{w})\right| \psi^{0}\left(\overrightarrow{D_{y}^{s} u_{\varepsilon}}\right)\left|D_{y}^{c} u_{\varepsilon}\right|\right) d x \\
& \quad \leq C\left\|\xi_{n}\right\|_{\infty} \int_{\{\bar{w} \geq a\}}\left(\int_{\mathbb{R}^{N}}\left(\left|u_{\varepsilon}-\bar{w}\right|\right) \chi_{[\bar{w}(x), \bar{w}(x)+\varepsilon]}(w)\left|D_{y}^{c} w\right|\right) d x \\
& \quad \leq C \varepsilon\left\|\xi_{n}\right\|_{\infty} \int_{\{\bar{w} \geq a\}}\left(\int_{\mathbb{R}^{N}} \chi_{[\bar{w}(x), \bar{w}(x)+\varepsilon]}(u)\left|D_{y}^{c} w\right|\right) d x \\
& \quad \leq C \varepsilon\left\|\xi_{n}\right\|_{\infty} \int_{\{\bar{w} \geq a\}}\left(\int_{\mathbb{R}^{N}} \chi_{[\bar{w}(x), \bar{w}(x)+\varepsilon]}(u)\left|D_{y}^{c} w\right|\right) d x \\
& \quad \leq \varepsilon O(\varepsilon)\left\|\xi_{n}\right\|_{\infty}|\{\bar{w} \geq a\}|,
\end{aligned}
$$

where we have reabsorbed in $o(\varepsilon)$ (it depends on $a$ ) the inner integral.

That is

$$
\frac{1}{\varepsilon}\left|T_{1,1}\left(I_{2,2}(s)\right)\right| \leq o(\varepsilon)\left\|\xi_{n}\right\|_{\infty}|\{\bar{w} \geq a\}| .
$$

Bound on $T_{1,2}\left(I_{2,2}(s)\right)$. For convenience, let us write

$$
J=J\left(u_{\varepsilon}, y\right)=\frac{1}{\left(u_{\varepsilon}\right)^{+}(y)-\left(u_{\varepsilon}\right)^{-}(y)} .
$$

Working in a similar way as before, we have

$$
\begin{aligned}
& \left|T_{1,2}\left(I_{2,2}(s)\right)\right| \\
& \leq \int_{\{\bar{w} \geq a\}} \int_{J_{u_{\varepsilon}}} J \xi_{n}\left(\int_{\left(u_{\varepsilon}\right)^{-(y)}}^{\left(u_{\varepsilon}\right)^{+}(y)} \mid T(s) \varphi^{\Phi}(s)-T\left(\bar{w}(x) \varphi^{\Phi}(\bar{w}(x) \mid d s) \psi^{0}\left(\overrightarrow{D_{y}^{s} u_{\varepsilon}}\right)\left|D_{y}^{j} u_{\varepsilon}\right| d x\right.\right. \\
& \leq C(a) \int_{\{\bar{w} \geq a\}} \int_{J_{u_{\varepsilon}}} J \xi_{n}\left(\int_{\left(u_{\varepsilon}\right)^{-}(y)}^{\left(u_{\varepsilon}\right)^{+}(y)} \mid s-\bar{w}(x) d s\right) \psi^{0}\left(\overrightarrow{D_{y}^{s} u_{\varepsilon}}\right)\left|D_{y}^{j} u_{\varepsilon}\right| d x \\
& \leq C(a) \varepsilon\left\|\xi_{n}\right\|_{\infty} \int_{\{\bar{w} \geq a\}} \int_{J_{u_{\varepsilon}}} J\left(\int_{\left(u_{\varepsilon}\right)^{-}(y)}^{\left(u_{\varepsilon}\right)^{+}(y)} d s\right) \psi^{0}\left(\overrightarrow{\left.D_{y}^{s} u_{\varepsilon}\right)}\right)\left|D_{y}^{j} u_{\varepsilon}\right| d x \\
& \leq C(a) \varepsilon\left\|\xi_{n}\right\|_{\infty} \int_{\{\bar{w} \geq a\}}\left(\int_{J_{u_{\varepsilon}}}\left|D_{y}^{j} u_{\varepsilon}\right|\right) d x \\
& \leq \varepsilon o(\varepsilon)\left\|\xi_{n}\right\|_{\infty}|\{\bar{w} \geq a\}|,
\end{aligned}
$$

where we have reabsorbed in $o(\varepsilon)$ the constant $C(a)$ and the integral $\int_{J_{u_{\varepsilon}}}\left|D_{y}^{j} u_{\varepsilon}\right|$. 
We obtain that

$$
\frac{1}{\varepsilon} T_{1,2}\left(I_{2,2}(s)\right) \geq o(\varepsilon)\left\|\xi_{n}\right\|_{\infty}|\{\bar{w} \geq a\}| .
$$

Collecting all these facts, we obtain

$$
\frac{1}{\varepsilon} T_{1}\left(I_{2,2}(s)\right) \geq o(\varepsilon)\left\|\xi_{n}\right\|_{\infty}|\{\bar{w} \geq a\}| .
$$

In a similar way we prove that

$$
\frac{1}{\varepsilon} T_{2}\left(I_{2,2}(s)\right) \geq o(\varepsilon)\left\|\xi_{n}\right\|_{\infty}|\{w \geq a\}|
$$

Hence

$$
\frac{1}{\varepsilon} I_{2,2}(s) \geq o(\varepsilon)\left\|\xi_{n}\right\|_{\infty}(|\{w \geq a\}|+|\{\bar{w} \geq a\}|) .
$$

Then, combining this inequality and (3.48), it follows that

$$
\frac{1}{\varepsilon} I_{2,2} \geq o(\varepsilon)\left\|\xi_{n}\right\|_{\infty}(|\{w \geq a\}|+|\{\bar{w} \geq a\}|) .
$$

Hence, with the estimates of all terms of $I_{2}$, we obtain (3.46) (where we have written $o(\varepsilon)\left\|\xi_{n}\right\|_{\infty}(|\{w \geq a\}|+|\{\bar{w} \geq a\}|)$ as $\left.o(\varepsilon)\right)$.

Step 3. Passing to the limit and final steps. Therefore, dividing (3.45) by $\varepsilon$, and letting $\varepsilon \rightarrow 0$ and $n \rightarrow \infty$ in this order we obtain

$$
\begin{aligned}
\int_{\mathbb{R}^{N}} & (u(x) T(w(x))-\bar{u}(x) T(\bar{w}(x))) \operatorname{sign}_{0}^{+}(w(x)-\bar{w}(x)) d x \\
\left.\leq \int_{\mathbb{R}^{N}}(v(x) T(w(x)))-\bar{v}(x) T(\bar{w}(x))\right) & \operatorname{sign}_{0}^{+}(w(x)-\bar{w}(x)) d x \\
& -\int_{\mathbb{R}^{N}} \operatorname{div}(\overline{\mathbf{z}}) T(\bar{w}(x)) d x .
\end{aligned}
$$

As above, let us skip the argument $x$ in the expressions below. Since $\operatorname{sign}_{0}^{+}(w(x)-\bar{w}(x))=\operatorname{sign}_{0}^{+}(u-\bar{u})$, letting $a \rightarrow 0^{+}$, we obtain

$$
\begin{aligned}
& \int_{\mathbb{R}^{N}}\left(u T_{0, b}(w)-\bar{u} T_{0, b}(\bar{w})\right) \operatorname{sign}_{0}^{+}(u-\bar{u}) d x \\
& \quad \leq \int_{\mathbb{R}^{N}}\left(v T_{0, b}(w)-\bar{v} T_{0, b}(\bar{w})\right) \operatorname{sign}_{0}^{+}(u-\bar{u}) d x-\int_{\mathbb{R}^{N}} \operatorname{div}(\overline{\mathbf{z}}) T_{0, b}(\bar{w}) d x
\end{aligned}
$$


Dividing by $b>0$, and letting $b \rightarrow 0^{+}$and using that $\frac{1}{b} T_{0, b}(w) \rightarrow$ $\chi_{\{w>0\}}=\chi_{\{u>0\}}$, we obtain

$$
\begin{aligned}
& \int_{\mathbb{R}^{N}}\left(u \chi_{\{u>0\}}-\bar{u} \chi_{\{\bar{u}>0\}}\right) \operatorname{sign}_{0}^{+}(u-\bar{u}) d x \\
& \leq \int_{\mathbb{R}^{N}}\left(v \chi_{\{u>0\}}-\bar{v} \chi_{\{\bar{u}>0\}}\right) \operatorname{sign}_{0}^{+}(u-\bar{u}) d x \\
& \quad-\int_{\mathbb{R}^{N}} \operatorname{div}(\overline{\mathbf{z}}) \chi_{\{\bar{u}>0\}} d x .
\end{aligned}
$$

We claim now that

$$
v=0 \text { a.e. on }\{u=0\} \quad \text { and } \quad \bar{v}=0 \text { a.e. on }\{\bar{u}=0\} \text {. }
$$

Let $0 \leq \phi \in \mathcal{D}\left(\mathbb{R}^{N}\right)$ be and $a>0, \varepsilon>0$. Multiplying $v-u=-\operatorname{div}(\mathbf{z})$ in $\mathcal{D}^{\prime}\left(\mathbb{R}^{N}\right)$ by $T_{a, a+\varepsilon}^{a}(w) \phi$ and integrating by parts, we have

$$
\begin{aligned}
\int_{\mathbb{R}^{N}} & (v-u) T_{a, a+\varepsilon}^{a}(w) \phi d x \\
& =\int_{\mathbb{R}^{N}} \phi\left(\mathbf{z} \cdot D T_{a, a+\varepsilon}^{a}(w)\right)+\int_{\mathbb{R}^{N}} \mathbf{z} \cdot \nabla \phi T_{a, a+\varepsilon}^{a}(w) d x \\
& \geq \int_{\mathbb{R}^{N}} \mathbf{z} \cdot \nabla \phi T_{a, a+\varepsilon}^{a}(w) d x .
\end{aligned}
$$

Dividing by $\varepsilon$ and letting $\varepsilon \rightarrow 0^{+}$, we get

$$
\int_{\mathbb{R}^{N}}(v-u) \chi_{\{w>a\}} \phi d x \geq \int_{\mathbb{R}^{N}} \mathbf{z} \cdot \nabla \phi \chi_{\{w>a\}} d x .
$$

Hence

$$
\begin{gathered}
\int_{\mathbb{R}^{N}}(v-u) \chi_{\{w \leq a\}} \phi d x=\int_{\mathbb{R}^{N}}(v-u) \phi d x-\int_{\mathbb{R}^{N}}(v-u) \chi_{\{w>a\}}(x) \phi d x \\
\leq \int_{\mathbb{R}^{N}}(v-u) \phi d x-\int_{\mathbb{R}^{N}} \mathbf{z} \cdot \nabla \phi \chi_{\{w>a\}} d x=\int_{\mathbb{R}^{N}} \mathbf{z} \cdot \nabla \phi \chi_{\{w \leq a\}} d x .
\end{gathered}
$$

Then, letting $a \rightarrow 0^{+}$, since $\mathbf{z}=0$ in $\{u=0\}=\{w=0\}$, we have

$$
\int_{\mathbb{R}^{N}} v \chi_{\{u=0\}} \phi d x=\int_{\mathbb{R}^{N}}(v-u) \chi_{\{u=0\}} \phi d x \leq 0,
$$


for all $0 \leq \phi \in \mathcal{D}\left(\mathbb{R}^{N}\right)$. Hence $v \chi_{\{u=0\}}=0$ a.e. in $\mathbb{R}^{N}$. Similarly, $\bar{v} \chi_{\{\bar{u}=0\}}=0$ a.e. in $\mathbb{R}^{N}$ and (3.52) holds.

On the other hand, by (3.52), we have

$$
\begin{aligned}
\int_{\mathbb{R}^{N}} \operatorname{div}(\overline{\mathbf{z}}) \chi_{\{\bar{u}>0\}} d x & =\int_{\mathbb{R}^{N}}(\bar{u}-\bar{v}) \chi_{\{\bar{u}>0\}} d x \\
& =\int_{\mathbb{R}^{N}}(\bar{u}-\bar{v}) d x=\int_{\mathbb{R}^{N}} \operatorname{div}(\overline{\mathbf{z}}) d x=0 .
\end{aligned}
$$

Then, from (3.51), it follows that

$$
\begin{aligned}
& \int_{\mathbb{R}^{N}}\left(u \chi_{\{u>0\}}-\bar{u} \chi_{\{\bar{u}>0\}}\right) \operatorname{sign}_{0}^{+}(u-\bar{u}) d x \\
& \leq \int_{\mathbb{R}^{N}}\left(v \chi_{\{u>0\}}-\bar{v} \chi_{\{\bar{u}>0\}}\right) \operatorname{sign}_{0}^{+}(u-\bar{u}) d x .
\end{aligned}
$$

Hence, using (3.52), we obtain

$$
\int_{\mathbb{R}^{N}}(u-\bar{u})^{+} d x \leq \int_{\mathbb{R}^{N}}(v-\bar{v}) \operatorname{sign}_{0}^{+}(u-\bar{u}) d x \leq \int_{\mathbb{R}^{N}}(v-\bar{v})^{+} d x .
$$

This concludes the proof of the theorem.

\section{Semigroup solution}

In this section we shall associate an accretive operator in $L^{1}\left(\mathbb{R}^{N}\right)$ to the formal differential expression $-\operatorname{div} \mathbf{a}(u, \nabla \Phi(u))$.

Definition 4.1. $(u, v) \in B$ if and only if $0 \leq u \in L^{1}\left(\mathbb{R}^{N}\right) \cap L^{\infty}\left(\mathbb{R}^{N}\right)$, $\Phi(u) \in T B V_{r}^{+}\left(\mathbb{R}^{N}\right), 0 \leq v \in L^{1}\left(\mathbb{R}^{N}\right) \cap L^{\infty}\left(\mathbb{R}^{N}\right)$ and $\mathbf{a}(u, \nabla \Phi(u)) \in$ $X_{1}\left(\mathbb{R}^{N}\right)$ satisfies:

$$
\begin{gathered}
v=-\operatorname{div} \mathbf{a}(u, \nabla \Phi(u)) \quad \text { in } \mathcal{D}^{\prime}\left(\mathbb{R}^{N}\right) \\
h_{S: T}^{\Phi}\left(\Phi(u), D T_{a, b}(\Phi(u))\right) \leq\left(\mathbf{a}(u, \nabla \Phi(u)), D J_{T^{\prime}}(\Phi(u))\right) \\
\quad \text { as measures } \forall(S, T) \in \mathcal{T S U B} .
\end{gathered}
$$

Recall that we assume that $\Phi^{-1} \in W^{1, \infty}([a, b])$ for all $0<a<b$. Hence $u \in T B V_{r}^{+}\left(\mathbb{R}^{N}\right)$.

Proposition 4.2. Assume we are under assumptions $(\mathrm{H})$. Let $v \in$ $\left(L^{1}\left(\mathbb{R}^{N}\right) \cap L^{\infty}\left(\mathbb{R}^{N}\right)\right)^{+}$. Then

(i) $u \in\left(L^{1}\left(\mathbb{R}^{N}\right) \cap L^{\infty}\left(\mathbb{R}^{N}\right)\right)^{+}$is an entropy solution of (3.1) if and only if $u=(I+B)^{-1} v$, i.e., if $(u, v-u) \in B$.

(ii) $u \ll v$, i.e., $\int_{\mathbb{R}^{N}} j(u) d x \leq \int_{\mathbb{R}^{N}} j(v) d x$ for any $j \in J_{0}$. 
(iii) $B$ is accretive in $L^{1}\left(\mathbb{R}^{N}\right),\left(L^{1}\left(\mathbb{R}^{N}\right) \cap L^{\infty}\left(\mathbb{R}^{N}\right)\right)^{+} \subset \operatorname{Range}(I+B)$ and $D(B)$ is dense in $L^{1}\left(\mathbb{R}^{N}\right)^{+}$.

Statement (i) follows directly from the definitions. Statement (ii) can be proved either directly using test functions $p_{a, b}(u)=p\left(T_{a, b}(u)\right)=$ $p\left(\Phi^{-1}\left(T_{\Phi(a), \Phi(b)}(\Phi(u))\right)\right), p \in \mathcal{P}_{0}, 0<a<b$, or obtained from Theorem 3.12 using the uniqueness of entropy solutions. Statement (iii) follows as in Proposition 5.3 in [5].

From Proposition 4.2 , if we denote by $\mathcal{B}$ the closure in $L^{1}\left(\mathbb{R}^{N}\right)$ of the operator $B$, it follows that $\mathcal{B}$ is accretive in $L^{1}\left(\mathbb{R}^{N}\right)$, it satisfies the comparison principle, and verifies the range condition $\overline{D(\mathcal{B})} L^{1}\left(\mathbb{R}^{N}\right)=$ $L^{1}\left(\mathbb{R}^{N}\right)^{+} \subset \operatorname{Range}(I+\lambda \mathcal{B})$ for all $\lambda>0$. Therefore, according to the Crandall-Liggett Theorem [16], [35], for any $0 \leq u_{0} \in L^{1}\left(\mathbb{R}^{N}\right)$ there exists a unique mild solution $u \in C\left([0, T] ; L^{1}\left(\mathbb{R}^{N}\right)\right)$ of the abstract Cauchy problem

$$
u^{\prime}(t)+\mathcal{B} u(t) \ni 0, \quad u(0)=u_{0} .
$$

Moreover, $u(t)=T(t) u_{0}$ for all $t \geq 0$, where $(T(t))_{t \geq 0}$ is the semigroup in $L^{1}\left(\mathbb{R}^{N}\right)^{+}$generated by the Crandall-Liggett's exponential formula, i.e.,

$$
T(t) u_{0}=\lim _{n \rightarrow \infty}\left(I+\frac{t}{n} \mathcal{B}\right)^{-n} u_{0} .
$$

Finally, the comparison principle also holds for $T(t)$, i.e., if $u_{0}, \bar{u}_{0} \in$ $L^{1}\left(\mathbb{R}^{N}\right)^{+}$, we have the estimate

$$
\left\|\left(T(t) u_{0}-T(t) \bar{u}_{0}\right)^{+}\right\|_{1} \leq\left\|\left(u_{0}-\bar{u}_{0}\right)^{+}\right\|_{1} .
$$

Remark 4.3. Since, by Proposition 4.2(iii), $\left(L^{1}\left(\mathbb{R}^{N}\right) \cap L^{\infty}\left(\mathbb{R}^{N}\right)\right)^{+} \subset$ Range $(I+B)$, using Proposition 4.2(ii) we have that

$$
T(t) u_{0} \in\left(L^{1}\left(\mathbb{R}^{N}\right) \cap L^{\infty}\left(\mathbb{R}^{N}\right)\right)^{+} \forall t \geq 0, \forall u_{0} \in\left(L^{1}\left(\mathbb{R}^{N}\right) \cap L^{\infty}\left(\mathbb{R}^{N}\right)\right)^{+} .
$$

\section{Existence and uniqueness of solutions of the evolution problem}

In this section we give the concept of entropy solution for the Cauchy problem (1.1) and we state the existence and uniqueness result for this type of solution.

By $L_{w}^{1}\left(0, T ; B V\left(\mathbb{R}^{N}\right)\right)$ we denote the space of weakly* measurable functions $\omega:[0, T] \rightarrow B V\left(\mathbb{R}^{N}\right)$ (i.e., $t \in[0, T] \rightarrow\langle\omega(t), \phi\rangle$ is measurable for every $\phi$ in the predual of $\left.B V\left(\mathbb{R}^{N}\right)\right)$ such that $\int_{0}^{T}\|\omega(t)\|_{B V} d t<\infty$. Observe that, since $B V\left(\mathbb{R}^{N}\right)$ has a separable predual (see [1]), it follows easily that the map $t \in[0, T] \rightarrow\|w(t)\|_{B V}$ is measurable. By 
$L_{\text {loc }, w}^{1}\left(0, T ; B V\left(\mathbb{R}^{N}\right)\right)$ we denote the space of weakly* measurable functions $\omega:[0, T] \rightarrow B V\left(\mathbb{R}^{N}\right)$ such that the map $t \in[0, T] \rightarrow\|\omega(t)\|_{B V}$ is in $L_{\text {loc }}^{1}((0, T))$.

Our concept of solution for problem (1.1) is the following.

Definition 5.1. Let $u_{0} \in\left(L^{1}\left(\mathbb{R}^{N}\right) \cap L^{\infty}\left(\mathbb{R}^{N}\right)\right)^{+}$. A measurable function $u:(0, T) \times \mathbb{R}^{N} \rightarrow \mathbb{R}$ is an entropy solution of $(1.1)$ in $Q_{T}=(0, T) \times \mathbb{R}^{N}$ if $u \in C\left([0, T], L^{1}\left(\mathbb{R}^{N}\right)^{+}\right), T_{a, b}(\Phi(u(\cdot)))-a \in L_{\text {loc }, w}^{1}\left(0, T ; B V\left(\mathbb{R}^{N}\right)\right)$ for all $0<a<b \leq \infty$, and

(i) $\mathbf{a}(u(t), \nabla \Phi(u(t))) \in L^{1}\left(\mathbb{R}^{N}\right) \cap L^{\infty}\left(\mathbb{R}^{N}\right)$ for a.e. $t \in(0, T)$,

(ii) $u_{t}=\operatorname{div} \mathbf{a}(u(t), \nabla \Phi(u(t)))$ in $\mathcal{D}^{\prime}\left(Q_{T}\right)$,

(iii) $u(0)=u_{0}$, and

(iv) the following inequality is satisfied

$$
\begin{aligned}
\int_{0}^{T} \int_{\mathbb{R}^{N}} \phi h_{S: T}^{\Phi}\left(\Phi(u), D T_{a, b}(\Phi(u))\right) d t \\
\quad+\int_{0}^{T} \int_{\mathbb{R}^{N}} \phi h_{T: S}^{\Phi}\left(\Phi(u), D S_{c, d}(\Phi(u))\right) d t \\
\leq \int_{0}^{T} \int_{\mathbb{R}^{N}} J_{T \circ \Phi \text { } \circ \Phi}(u(t)) \phi^{\prime}(t) d x d t \\
-\int_{0}^{T} \int_{\mathbb{R}^{N}} \mathbf{a}(u(t), \nabla \Phi(u(t))) \cdot \nabla \phi T(\Phi(u(t))) S(\Phi(u(t))) d x d t
\end{aligned}
$$

for truncatures $(S, T) \in \mathcal{T} \mathcal{S U B}$ with $T=\tilde{T} \circ T_{a, b}, S=\tilde{S} \circ S_{c, d}$, and any smooth function $\phi$ of compact support, in particular of the form $\phi(t, x)=\phi_{1}(t) \rho(x), \phi_{1} \in \mathcal{D}((0, T)), \rho \in \mathcal{D}\left(\mathbb{R}^{N}\right)$.

We observe that the functions that appear in (5.1) are measurable. For a proof we refer to Proposition 6.1 in [9].

Remark 5.2. The notion of entropy subsolution of (1.1) can be defined by replacing the identity in condition (ii) by $\leq$ or just by omitting it, since the inequality $\leq$ in (ii) is implied by (iv). To define the notion of entropy supersolution of (1.1) we need to use test functions $(S, T) \in \mathcal{T} \mathcal{S U P \mathcal { R }}$ in (iv).

We have the following existence and uniqueness result. 
Theorem 5.3. Assume we are under assumptions $(\mathrm{H})$. Then, for any initial datum $0 \leq u_{0} \in L^{1}\left(\mathbb{R}^{N}\right) \cap L^{\infty}\left(\mathbb{R}^{N}\right)$ there exists a unique entropy solution $u$ of (1.1) in $Q_{T}=(0, T) \times \mathbb{R}^{N}$ for every $T>0$ such that $u(0)=$ $u_{0}$. Moreover, if $u(t), \bar{u}(t)$ are the entropy solutions corresponding to initial data $u_{0}, \bar{u}_{0} \in L^{1}\left(\mathbb{R}^{N}\right)^{+}$, respectively, then

$$
\left\|(u(t)-\bar{u}(t))^{+}\right\|_{1} \leq\left\|\left(u_{0}-\bar{u}_{0}\right)^{+}\right\|_{1} \quad \text { for all } t \geq 0 .
$$

The proof of the theorem follows the steps of the analogous theorem in $[\mathbf{6}],[\mathbf{2 9}]$. The existence follows by proving that the semigroup solution constructed in Section 4 is an entropy solution. The uniqueness result follows using Kružkov's doubling variables technique [42] for this type of problems as used in $[\mathbf{6}],[\mathbf{2 9}]$. The adaptation of these techniques can be done easily taking into account the proofs of the stationary case. Thus, we shall omit the details. As a consequence of the uniqueness of entropy solutions we have that semigroup and entropy solutions coincide.

Remark 5.4. If we assume that $u \in L_{w}^{1}\left(0, T, B V\left(\mathbb{R}^{N}\right)\right)$ and is a entropy subsolution and $\bar{u} \in L_{w}^{1}\left(0, T, B V\left(\mathbb{R}^{N}\right)\right)$ an entropy supersolution, then $\left\|(u(t)-\bar{u}(t))^{+}\right\|_{1} \leq\left\|\left(u_{0}-\bar{u}_{0}\right)^{+}\right\|_{1}$. This can be proved using the test functions $T=1, S_{\varepsilon, l}(\bar{z})=T_{\varepsilon}(\bar{z}-l)^{+}, S_{\varepsilon}^{l}(\bar{z})=T_{\varepsilon}(\bar{z}-l)^{-}=-T_{\varepsilon}(l-\bar{z})^{+}$, $\varepsilon>0, l \geq 0$. We are not able to prove this result when only $T_{a, \infty}(u)-$ $a, T_{a, \infty}(\bar{u})-a \in L_{w}^{1}\left(0, T, B V\left(\mathbb{R}^{N}\right)\right)$ for any $a>0$.

Remark 5.5. We observe that $u(t) \in B V\left(\mathbb{R}^{N}\right)$ for any $t>0$ if $u_{0} \in$ $B V\left(\mathbb{R}^{N}\right)$. Indeed, let $\tau_{h} u_{0}(x)=u_{0}(x+h), h \in \mathbb{R}^{N}$. By (5.2) we have

$$
\left\|u(t)-\tau_{h} u(t)\right\|_{1} \leq\left\|u_{0}-\tau_{h} u_{0}\right\|_{1} \quad \forall t>0 .
$$

Since $u_{0} \in B V\left(\mathbb{R}^{N}\right)$ we deduce that $u(t) \in B V\left(\mathbb{R}^{N}\right)$ for all $t>0$ and $\|u(t)\|_{B V} \leq\left\|u_{0}\right\|_{B V}$. Clearly $u \in L_{w}^{1}\left(0, T ; B V\left(\mathbb{R}^{N}\right)\right)$.

\section{Flux limited generalized porous media equations}

$(\mathrm{H})_{\Phi, \Lambda}$ We assume that $\Phi:[0, \infty) \rightarrow[0, \infty)$ is a continuous strictly increasing function such that $\Phi(0)=0$ and $\Phi, \Phi^{-1} \in W^{1, \infty}([a, b])$ for any $0<a<b$. Let $\Lambda:[0, \infty) \rightarrow[0, \infty)$ be a continuous function such that $\Lambda(0)=0$ and $\Lambda(z)>0$ for all $z>0$. We assume that $\Lambda(z)=\widetilde{\Lambda}\left(z^{\bar{m}}\right)$ where $\widetilde{\Lambda}(z) \geq c_{0} z$ for some $c_{0}>0$ and all $z \geq 0$ and $\widetilde{\Lambda} \in W_{\text {loc }}^{1, \infty}([0, \infty)$ and $\bar{m} \geq 1$. 
Let us consider the diffusion equation

$$
u_{t}=\alpha \operatorname{div}\left(\frac{\Lambda(u) \nabla \Phi(u)}{\sqrt{1+\beta|\nabla \Phi(u)|^{2}}}\right),
$$

where $\beta>0$. Let $f(z, \xi)=\frac{1}{\beta} \Lambda(z) \sqrt{1+\beta|\xi|^{2}}$ be the Lagrangian associated to (6.1). Since $f$ satisfies the assumptions of Subsection 3.1, by Theorem 5.3 there is a unique entropy solution $u(t)$ of $(6.1)$ for any initial condition $u_{0} \in\left(L^{1}\left(\mathbb{R}^{N}\right) \cap L^{\infty}\left(\mathbb{R}^{N}\right)\right)^{+}$.

6.1. Time regularity of $\boldsymbol{u}$. Our purpose in this section is to prove that $u_{t}(t)$ is a Radon measure for any $t>0$, assuming certain conditions on $u_{0}$. For that we follow the same approach as in [30] (see also [7]) which is based on semigroup theory. We use the basic result that if the initial condition $u_{0}$ is in the domain of $\mathcal{B}$ (see Section 4 ), then $\left\|u_{t}(t)\right\|_{1} \leq$ $\left\|\mathcal{B} u_{0}\right\|_{1}[\mathbf{3 5}]$. To use this result, we give in Lemma 6.1 a set of conditions on $u_{0}$ that guarantee that it can be approximated by $u_{0 n} \in\left(L^{1}\left(\mathbb{R}^{N}\right) \cap\right.$ $\left.L^{\infty}\left(\mathbb{R}^{N}\right)\right)^{+}$in the domain of $\mathcal{B}$ with $\left\|\mathcal{B} u_{0 n}\right\|_{1}$ bounded. As a consequence we derive that if $u_{n}(t)$ is the entropy solution of $(6.1)$ with $u_{n}(0)=u_{0 n}$, then $\left\|u_{n t}(t)\right\|_{1} \leq\left\|\mathcal{B} u_{0 n}\right\|_{1}$ for any $n \geq 1$ and any $t>0$. Letting $n \rightarrow \infty$ we deduce that $u_{t}(t)$ is a Radon measure for any $t>0$.

As a consequence we have that $S(u) \in B V\left([\tau, T] \times \mathbb{R}^{N}\right)$ for any $0<$ $\tau<T$ and any truncature $S \in \mathcal{T}_{r}$. If, in addition, $u_{0} \in B V\left(\mathbb{R}^{N}\right)$, then $u \in B V\left([\tau, T] \times \mathbb{R}^{N}\right)$ for any $0<\tau<T$. We cannot expect a higher regularity in general, due to the existence of moving discontinuity fronts for this type of equations [8], although solutions may be smooth outside the discontinuity fronts, but this is an open question.

We believe that the result that $u \in B V\left([\tau, T] \times \mathbb{R}^{N}\right)$ for any $0<\tau<T$ should be true for any $u_{0} \in B V\left(\mathbb{R}^{N}\right)$, although we do not have a proof of it in the general case. The conditions on $u_{0}$ given in Lemma 6.1 are of technical nature, in order to use the previously described semigroup approach.

As shown in $[\mathbf{3 0}]$ the fact that $u \in B V\left([\tau, T] \times \mathbb{R}^{N}\right)$ for any $0<$ $\tau<T$ permits to identify the Rankine Hugoniot condition, to give a more concrete characterization of the entropy conditions on the jump set of $u$ and to compute the speed of the moving discontinuity fronts (the existence of such fronts and other regularity results will be the object of a subsequent paper [31]). This will be the purpose of Subsection 6.2.

Let us finally mention that no classical regularity result seems to work in the present case. In particular, local estimates of the gradient have to take into account that a moving discontinuity front may arrive at a region where the initial condition was regular, coming from a jump located 
somewhere else. This explains why we have decided to use semigroup methods.

Let us first proceed with some formal computations. Let $v=\Phi\left(u_{0}\right)$. Then

$$
\begin{aligned}
\operatorname{div}\left(\frac{\Lambda\left(u_{0}\right) \nabla \Phi\left(u_{0}\right)}{\sqrt{1+\beta\left|\nabla \Phi\left(u_{0}\right)\right|^{2}}}\right)= & \Lambda\left(u_{0}\right) \operatorname{div}\left(\frac{\nabla v}{\sqrt{1+\beta|\nabla v|^{2}}}\right) \\
& +\nabla \Lambda\left(u_{0}\right) \cdot \frac{\nabla v}{\sqrt{1+\beta|\nabla v|^{2}}} \\
= & \Lambda\left(u_{0}\right) \operatorname{div}\left(\frac{\nabla v}{\sqrt{1+\beta|\nabla v|^{2}}}\right) \\
& +\left(\Lambda \circ \Phi^{-1}\right)^{\prime}(v) \frac{|\nabla v|^{2}}{\sqrt{1+\beta|\nabla v|^{2}}} .
\end{aligned}
$$

Clearly, the assumptions $(\mathrm{H})_{\Phi, \Lambda}$ imply that if $u_{0} \in\left(L^{1}\left(\mathbb{R}^{N}\right) \cap L^{\infty}\left(\mathbb{R}^{N}\right)\right)^{+}$, then $\Lambda\left(u_{0}\right) \in L^{\infty}\left(\mathbb{R}^{N}\right)$.

To bound the second term we assume either

$$
(\mathrm{H})_{\Phi, \Lambda}^{(1)} \quad \Phi \in C^{2}(0, \infty), \Lambda \circ \Phi^{-1} \in W_{\mathrm{loc}}^{1, \infty}([0, \infty),
$$

or

$$
(\mathrm{H})_{\Phi, \Lambda}^{(2)} \quad \Phi, \Phi^{\prime} \in W_{\text {loc }}^{1, \infty}([0, \infty) .
$$

Notice that, if $(\mathrm{H})_{\Phi, \Lambda}^{(1)}$ holds, then $\left(\Lambda \circ \Phi^{-1}\right)^{\prime}(v) \in L^{\infty}\left(\mathbb{R}^{N}\right)$.

Lemma 6.1. Assume that $\Phi, \Lambda$ satisfy the assumptions $(\mathrm{H})_{\Phi, \Lambda}$. Assume also that either $(\mathrm{H})_{\Phi, \Lambda}^{(1)}$ or $(\mathrm{H})_{\Phi, \Lambda}^{(2)}$ hold. Let $u_{0} \in L^{1}\left(\mathbb{R}^{N}\right) \cap L^{\infty}\left(\mathbb{R}^{N}\right)$, $u_{0}(x) \geq 0$. Let $\Gamma_{i}, i=0, \ldots, \ell$, be the boundaries of bounded open sets of class $C^{1,1}$ (so that the distance function is also $C^{1,1}$ in a neighborhood of it). Assume that

(i) $\operatorname{dist}\left(\Gamma_{i}, \Gamma_{j}\right)>0$ for any $i \neq j$.

(ii) $u_{0} \in W^{2,1}\left(\mathbb{R}^{N} \backslash \cup_{i=0}^{\ell} \Gamma_{i}\right)$ and $\nabla u_{0} \in L^{\infty}\left(\mathbb{R}^{N} \backslash \cup_{i=0}^{\ell} \Gamma_{i}\right)$.

(iii) $u_{0}$ is discontinuous in $\Gamma_{i}$ with $\left.u_{0}^{+}\right|_{\Gamma_{i}}>c_{i}+\delta_{i}>c_{i}-\delta_{i}>\left.u_{0}^{-}\right|_{\Gamma_{i}}$, $i=0, \ldots, \ell$.

(iv) $u_{0}$ is either 0 or is bounded away from zero in any connected component of $\mathbb{R}^{N} \backslash \cup_{i=0}^{\ell} \Gamma_{i}$.

(v) Let $d(x)=d\left(x, \cup_{i=0}^{\ell} \Gamma_{i}\right), d_{i}(x)=d\left(x, \Gamma_{i}\right), i \in\{0, \ldots, \ell\}, x \in \mathbb{R}^{N}$. For each $i \in\{0, \ldots, \ell\}$ such that $\left.u_{0}^{-}\right|_{\Gamma_{i}} \neq 0$ there is some $\eta>0$ such that $(1+\eta)\left|\nabla d \cdot \nabla u_{0}\right| \leq\left|\nabla u_{0}\right|$ in a region $\left\{x \in \mathbb{R}^{N}: 0<\right.$ $\left.d_{i}(x)<\rho,\left(u_{0}-c_{i}\right) \nabla d \cdot \nabla u_{0}<0\right\}$ for some $\rho>0$. 
Then, there exist functions $u_{0 n} \in L^{1}\left(\mathbb{R}^{N}\right) \cap L^{\infty}\left(\mathbb{R}^{N}\right), u_{0 n} \geq 0$, such that $u_{0 n} \rightarrow u_{0}$ in $L^{1}\left(\mathbb{R}^{N}\right), u_{0 n} \in \operatorname{Dom}(\mathcal{B})$ and $\left\|\mathcal{B} u_{0 n}\right\|_{1}$ is bounded.

Conditions (i)-(v) say that $u_{0}$ is a piecewise $W^{2,1}$ function with discontinuities located at a set of $C^{1,1}$ hypersurfaces $\Gamma_{i}$. We also assume that $\nabla u_{0}$ is bounded outside $\cup_{i=0}^{\ell} \Gamma_{i}$. While the $W^{2,1}$ condition seems to be necessary to get the result of the lemma, this condition seems too strong and is only motivated by technical reasons. The same can be said for conditions (iii) and (v), whose only purpose is to be able to smooth the graph of $v=\Phi\left(u_{0}\right)$ in such a way that the mean curvature of the approximating graphs remains bounded in $L^{1}\left(\mathbb{R}^{N}\right)$. Thus, although the lemma may be true under more general conditions the ones we give illustrate the result and have a clear geometric interpretation.

Finally, let us say that one can rephrase assumption (v) by saying that when the trace $\left.u_{0}^{-}\right|_{\Gamma_{i}}$ is bounded away from zero, and we are on the side corresponding to the upper (resp. lower) trace of $u_{0}$, the direction of the gradient of $u_{0}$ and the normal to $\Gamma_{i}$ are not aligned near the points where $u_{0}$ is increasing (resp. decreasing) towards $\Gamma_{i}$.

The assumptions of the lemma permit to prove that $u$ can be approximated by functions $u_{0 n} \in L^{1}\left(\mathbb{R}^{N}\right) \cap L^{\infty}\left(\mathbb{R}^{N}\right), u_{0 n} \geq 0$, such that

$u_{0 n}$ is uniformly bounded in $L^{\infty}\left(\mathbb{R}^{N}\right)$,

$$
\begin{aligned}
& u_{0 n} \rightarrow u_{0} \text { in } L^{1}\left(\mathbb{R}^{N}\right), \\
& u_{0 n} \in W^{2,1}\left(\mathbb{R}^{N}\right), \\
& \nabla u_{0 n} \text { is uniformly bounded in } L^{1}\left(\mathbb{R}^{N}\right) .
\end{aligned}
$$

If condition $(\mathrm{H})_{\Phi, \Lambda}^{(2)}$ holds, then $v_{n}=\Phi\left(u_{0 n}\right) \in L^{1}\left(\mathbb{R}^{N}\right) \cap L^{\infty}\left(\mathbb{R}^{N}\right), v_{n} \geq$ 0 , and

$$
\begin{aligned}
& v_{n} \text { is uniformly bounded in } L^{\infty}\left(\mathbb{R}^{N}\right), \\
& v_{n} \rightarrow v \text { in } L^{1}\left(\mathbb{R}^{N}\right), \\
& v_{n} \in W^{2,1}\left(\mathbb{R}^{N}\right), \\
& \nabla v_{n} \text { is uniformly bounded in } L^{1}\left(\mathbb{R}^{N}\right) .
\end{aligned}
$$

In that case $\nabla \Lambda\left(u_{0 n}\right)=\Lambda^{\prime}\left(u_{0 n}\right) \nabla u_{0 n}$ is uniformly bounded in $L^{1}\left(\mathbb{R}^{N}\right)$. Hence

$$
\nabla \Lambda\left(u_{0 n}\right) \cdot \frac{\nabla v_{n}}{\sqrt{1+\beta\left|\nabla v_{n}\right|^{2}}}
$$

is also is uniformly bounded in $L^{1}\left(\mathbb{R}^{N}\right)$.

If $(\mathrm{H})_{\Phi, \Lambda}^{(1)}$ holds, and the other assumptions of the lemma hold, then $v:=\Phi\left(u_{0}\right)$ satisfies also conditions (ii)-(iii)-(iv)-(v). Moreover, as in [30], 
they permit to prove that $v$ can be approximated by functions $v_{n} \in$ $L^{1}\left(\mathbb{R}^{N}\right) \cap L^{\infty}\left(\mathbb{R}^{N}\right), v_{n} \geq 0$, such that (6.3) holds. The last condition of (6.3) implies that

$$
\frac{\left|\nabla v_{n}\right|^{2}}{\sqrt{1+\beta\left|\nabla v_{n}\right|^{2}}}
$$

is uniformly bounded in $L^{1}\left(\mathbb{R}^{N}\right)$.

Finally, as in [30], using the assumptions of the lemma we prove that

$$
\operatorname{div}\left(\frac{\nabla v_{n}}{\sqrt{1+\beta\left|\nabla v_{n}\right|^{2}}}\right) \quad \text { is uniformly bounded in } L^{1}(\Omega),
$$

in each connected component $\mathbb{R}^{N} \backslash \cup_{i=0}^{\ell} \Gamma_{i}$. The functions $u_{0 n}=\Phi^{-1}\left(v_{n}\right)$ satisfy the lemma.

Proposition 6.2. Assume that $\Phi, \Lambda$ satisfy the assumptions $(\mathrm{H})_{\Phi, \Lambda}$. Assume also that either $(\mathrm{H})_{\Phi, \Lambda}^{(1)}$ or $(\mathrm{H})_{\Phi, \Lambda}^{(2)}$ hold. Let $u_{0} \in\left(L^{1}\left(\mathbb{R}^{N}\right) \cap\right.$ $\left.L^{\infty}\left(\mathbb{R}^{N}\right)\right)^{+}$. Let $u(t)$ be the entropy solution of $(6.1)$ with $u(0)=u_{0}$. If $u_{0}$ satisfies the conditions of Lemma 6.1, then for any $t>0, u_{t}(t)$ is a finite Radon measure in $\mathbb{R}^{N}$. Moreover $\left\|u_{t}(t)\right\|_{\mathcal{M}\left(\mathbb{R}^{N}\right)} \leq C$ for some constant $C>0$ depending on $u_{0}$. In particular, $T(u) \in B V\left([\tau, T] \times \mathbb{R}^{N}\right)$ for any $\tau>0$ and any truncature $T \in \mathcal{T}_{r}$. If $u_{0} \in B V\left(\mathbb{R}^{N}\right)$, then $u \in B V\left([0, T] \times \mathbb{R}^{N}\right)$ for any $T>0$.

Proof: Let $u_{n}(t)$ be the solution of (6.1) such that $u_{n}(0)=u_{0 n}$. By the nonlinear semigroup theory (see for instance $[\mathbf{3 5}]$ or $[\mathbf{1 6}]$ ), we have $u_{n t} \in L^{1}\left(\mathbb{R}^{N}\right)$ and $\left\|u_{n t}\right\|_{1} \leq\left\|\mathcal{B} u_{0 n}\right\|_{1}$. Since $u_{n t} \rightarrow u_{t}$ in the distribution sense, then $u_{t}(t)$ is a finite Radon measure in $\mathbb{R}^{N}$ and $\left\|u_{t}(t)\right\|_{\mathcal{M}\left(\mathbb{R}^{N}\right)} \leq C$ for some constant $C>0$ depending on $u_{0}$. The last assertion follows from this and Remark 5.5.

Remark 6.3. Consider the flux limited porous media equation

$$
u_{t}=\alpha \operatorname{div}\left(\frac{u^{r} \nabla u^{m}}{\sqrt{1+\beta\left|\nabla u^{m}\right|^{2}}}\right),
$$

where $m>0, r \geq 1, \alpha, \beta>0$. In this case, $\Phi(u)=u^{m}, \Lambda(u)=u^{r}$ and $(\mathrm{H})_{\Phi, \Lambda}$ hold. If $m \geq 2$, then $(\mathrm{H})_{\Phi, \Lambda}^{(2)}$ holds. If $r \geq m$, then $(\mathrm{H})_{\Phi, \Lambda}^{(1)}$ holds. Proposition 6.2 can be applied to these cases.

6.2. Analysis of the entropy conditions. Assume that $u \in B V_{\text {loc }}\left((0, T) \times \mathbb{R}^{N}\right)$. Let us denote by $J_{u}$ the jump set of $u$ as a function of $(t, x)$. For any $t>0$, we denote by $J_{u(t)}$ the jump set of $u(t) \in B V_{\text {loc }}\left(\mathbb{R}^{N}\right)$. Let $\nu:=\nu_{u}=\left(\nu_{t}, \nu_{x}\right)$ be the unit normal to the 
jump set of $u$ so that $D_{t, x}^{j} u=\left.[u] \nu \mathcal{H}^{N}\right|_{J_{u}}$. We denote by $\nu^{J_{u(t)}}$ the unit normal to the jump set of $u(t)$ so that $D_{x}^{j} u(t)=\left.[u(t)] \nu^{J_{u(t)}} \mathcal{H}^{N-1}\right|_{J_{u(t)}}$. Using the notation of Section $2[u](t, x):=u^{+}(t, x)-u^{-}(t, x)$ denotes the jump of $u$ at $(t, x) \in J_{u}$ and $[u(t)](x):=u(t)^{+}(x)-u(t)^{-}(x)$ denotes the jump of $u(t)$ at the point $x \in J_{u(t)}$.

Let us recall the definition of the speed of the discontinuity set of $u[30]$.

Definition 6.4. Let $u \in B V_{\mathrm{loc}}\left((0, T) \times \mathbb{R}^{N}\right)$ and let $\mathbf{z} \in L^{\infty}([0, T] \times$ $\left.\mathbb{R}^{N}, \mathbb{R}^{N}\right)$ be such that $u_{t}=\operatorname{div} \mathbf{z}$ in $\mathcal{D}^{\prime}\left((0, T) \times \mathbb{R}^{N}\right)$. We define the speed of the discontinuity set of $u$ as $v(t, x)=\frac{\nu_{t}(t, x)}{\left|\nu_{x}(t, x)\right|} \mathcal{H}^{N}$-a.e. on $J_{u}$.

This definition has a sense since, when $u \in B V_{\text {loc }}\left((0, T) \times \mathbb{R}^{N}\right), \mathbf{z} \in$ $L^{\infty}\left([0, T] \times \mathbb{R}^{N}, \mathbb{R}^{N}\right)$, and $u_{t}=\operatorname{div} \mathbf{z}$ in $\mathcal{D}^{\prime}\left((0, T) \times \mathbb{R}^{N}\right)$, we have (see [30, Lemma 6.4])

$$
\mathcal{H}^{N}\left((t, x) \in J_{u}: \nu_{x}(t, x)=0\right)=0 .
$$

Proposition 6.5. Let $u \in B V_{\mathrm{loc}}\left((0, T) \times \mathbb{R}^{N}\right)$ and let $\mathbf{z} \in L^{\infty}([0, T] \times$ $\left.\mathbb{R}^{N}, \mathbb{R}^{N}\right)$ be such that $u_{t}=\operatorname{div} \mathbf{z}$ in $\mathcal{D}^{\prime}\left((0, T) \times \mathbb{R}^{N}\right)$. For $\mathcal{L}^{1}$ almost any $t>0$ we have

$$
[u(t)](x) v(t, x)=\left[\left[\mathbf{z} \cdot \nu^{J_{u(t)}}\right]\right]_{+-} \quad \mathcal{H}^{N-1} \text {-a.e. in } J_{u(t)},
$$

where $\left[\left[\mathbf{z} \cdot \nu^{J_{u(t)}}\right]\right]_{+-}$denotes the difference of traces from both sides of $J_{u(t)}$.

We call outer side the side of $J_{u(t)}$ where $\nu^{J_{u(t)}}$ is pointing to. Thus the outer trace is $u(t)=u(t)^{+}$. Notice that with this notation, the Rankine-Hugoniot condition is expressed in an invariant way.

Notice that we are working under the assumption that $\operatorname{div} \mathbf{z}(t)$ is a Radon measure in $\mathbb{R}^{N}$ for almost any $t>0$. In the case a notion of weak trace for the normal component of $\mathbf{z}$ has been given in Subsection 2.3 on the boundary of open sets with Lipschitz boundary. In the present context, we need to use the following lemma which justifies the subsequent definition. For its proof and more details on this subject, we refer to $[\mathbf{3 0}]$.

Lemma 6.6. Let $D_{1}, D_{2} \subseteq \mathbb{R}^{N}$ be two open sets with Lipschitz boundary and let $\boldsymbol{\eta} \in L^{\infty}\left(\mathbb{R}^{N}, \mathbb{R}^{N}\right)$ be such that $\operatorname{div} \boldsymbol{\eta}$ is a Radon measure in $\mathbb{R}^{N}$. If $A \subseteq \partial D_{1} \cap \partial D_{2}$ is a Borel set and $\nu^{D_{1}}=\nu^{D_{2}}$ a.e. on $A$, then $\left[\boldsymbol{\eta} \cdot \nu^{D_{1}}\right]=$ $\left[\boldsymbol{\eta} \cdot \nu^{D_{2}}\right] \mathcal{H}^{N-1}$ a.e. on $A$.

Definition 6.7. Let $B$ be a $(N-1)$-rectifiable Borel set oriented by the normal $\nu^{B}$ (thus, it may be covered by the boundaries of open sets 
with Lipschitz boundary). Let $\boldsymbol{\eta} \in L^{\infty}\left(\mathbb{R}^{N}, \mathbb{R}^{N}\right)$ be such that $\operatorname{div} \boldsymbol{\eta}$ is a Radon measure in $\mathbb{R}^{N}$. We define $\left[\boldsymbol{\eta} \cdot \nu^{B}\right]$ as the trace $\left[\boldsymbol{\eta} \cdot \nu^{D}\right]$ for any open set with Lipschitz boundary $D$ such that $B \subseteq \partial D$ and $\nu^{B}=\nu^{D}$.

We have just written $\nu^{B}=\nu^{D}$ in order to fix one of the two choices of the normal.

Our purpose is to express the notion of entropy solution of (6.1) as a set of inequalities that permit to give a more geometric interpretation on the jump set. Informally, one can say that jump discontinuities are fronts with a vertical contact angle moving at the speed given by RankineHugoniot condition. This will be proved in Proposition 6.9.

If $w \in B V\left((0, T) \times \mathbb{R}^{N}\right)$, or in $B V\left(\mathbb{R}^{N}\right)$, and $\mu$ is a Radon measure absolutely continuous with respect to $|D w|$, we denote by $\mu^{a c}, \mu^{c}, \mu^{j}$ the absolutely continuous parts of $\mu$ with respect to $\left|D^{a c} w\right|,\left|D^{c} w\right|,\left|D^{j} w\right|$, respectively.

Proposition 6.8. Let $u \in C\left([0, T] ; L^{1}\left(\mathbb{R}^{N}\right)\right) \cap B V_{\text {loc }}\left((0, T) \times \mathbb{R}^{N}\right)$. Assume that $u_{t}=\operatorname{div} \mathbf{z}$ in $\mathcal{D}^{\prime}\left((0, T) \times \mathbb{R}^{N}\right)$ where $\mathbf{z}=\mathbf{a}(u, \nabla \Phi(u))$. Then $u$ is an entropy solution of (1.1) if and only if for any $(T, S) \in \mathcal{T} \mathcal{S U B}$ (for any $(T, S) \in \mathcal{T} \mathcal{S U B} \cup \mathcal{T S U P \mathcal { R }}$ ) we have

$$
\begin{aligned}
h_{S: T}^{\Phi}(\Phi(u), D T(\Phi(u)))^{c}+h_{T: S}^{\Phi} & (\Phi(u), D S(\Phi(u)))^{c} \\
& \leq(\mathbf{z}(t, x) \cdot D(T(\Phi(u)) S(\Phi(u))))^{c}
\end{aligned}
$$

and for $\mathcal{L}^{1}$-almost any $t>0$ the inequality

$$
\begin{aligned}
& {\left[S T \varphi^{\Phi}(\Phi(u(t)))\right]_{+-}-\left[J_{T S \varphi^{\Phi^{\prime}}}(\Phi(u(t)))\right]_{+-}} \\
& \quad \leq-v\left[J_{T \circ \Phi S \circ \Phi}(u(t))\right]_{+-}+\left[\left[\mathbf{z}(t) \cdot \nu^{J_{u(t)}}\right] T(\Phi(u(t))) S(\Phi(u(t)))\right]_{+-}
\end{aligned}
$$

holds $\mathcal{H}^{N-1}$ a.e. on $J_{u(t)}$.

Let us assume that $u \in C\left([0, T] ; L^{1}\left(\mathbb{R}^{N}\right)\right)$ is an entropy solution of $(6.1)$ with $u(0)=u_{0} \in\left(L^{1}\left(\mathbb{R}^{N}\right) \cap L^{\infty}\left(\mathbb{R}^{N}\right)\right)^{+}$. We assume that $u \in B V_{\text {loc }}\left((0, T) \times \mathbb{R}^{N}\right)$. By Proposition 6.2 and Remark 5.5, this holds in particular if $u_{0} \in B V\left(\mathbb{R}^{N}\right)$ satisfies the assumptions of Proposition 6.2. We assume this for simplicity, although the more general case where $T_{a, b}(u)-a \in L_{w}^{1}\left((0, T), B V\left(\mathbb{R}^{N}\right)\right)$ for any $0<a<b$ can be treated along the lines below and using the results in $[\mathbf{1}]$ for $G B V$ functions.

Since $u_{t}=\operatorname{div} \mathbf{z}$, by Proposition 6.2 we have that $\operatorname{div} \mathbf{z}$ is a Radon measure in $[\tau, T] \times \mathbb{R}^{N}$ for any $\tau>0$ and $\operatorname{div} z(t)$ is a Radon measure in $\mathbb{R}^{N}$ for any $t>0$. Thus, the conditions of Propositions 6.5 and 6.8 hold. 
For the equation (6.1) $\varphi=\Lambda$ and the conditions (6.8) can be written as

$$
\begin{aligned}
& {\left[S T \Lambda^{\Phi}(\Phi(u(t)))\right]_{+-}-\left[J_{T S \Lambda^{\Phi^{\prime}}}(\Phi(u(t)))\right]_{+-}} \\
& \quad \leq-v\left[J_{T \circ \Phi S \circ \Phi}(u(t))\right]_{+-}+\left[\left[\mathbf{z}(t) \cdot \nu^{J_{u(t)}}\right] T(\Phi(u(t))) S(\Phi(u(t)))\right]_{+-}
\end{aligned}
$$

holds $\mathcal{H}^{N-1}$ a.e. on $J_{u(t)}$. Notice also that $J_{T S \Lambda^{\Phi^{\prime}}}(\Phi(z))=J_{T \circ \Phi S \circ \Phi \Lambda^{\prime}}(z)$, for any $z \geq 0$, hence

$$
\left[J_{T S \Lambda^{\Phi^{\prime}}}(\Phi(u(t)))\right]_{+-}=\left[J_{T \circ \Phi S \circ \Phi \Lambda^{\prime}}(u(t))\right]_{+--} .
$$

Proposition 6.9. Assume that $\Phi, \Lambda$ satisfy the assumptions $(\mathrm{H})_{\Phi, \Lambda}$. Assume also that $\Lambda$ is an increasing and convex function. Let $u \in$ $C\left([0, T] ; L^{1}\left(\mathbb{R}^{N}\right)\right)$ be the entropy solution of $(6.1)$ with $u(0)=u_{0} \in$ $\left(L^{1}\left(\mathbb{R}^{N}\right) \cap L^{\infty}\left(\mathbb{R}^{N}\right)\right)^{+}$. We assume that $u \in B V_{\text {loc }}\left((0, T) \times \mathbb{R}^{N}\right)$. Then the entropy conditions (6.9) hold if and only if

$$
\left[\mathbf{z} \cdot \nu^{J_{u(t)}}\right]_{+}=\Lambda\left(u^{+}(t)\right) \quad \text { and } \quad\left[\mathbf{z} \cdot \nu^{J_{u(t)}}\right]_{-}=\Lambda\left(u^{-}(t)\right) .
$$

Moreover the velocity of the discontinuity fronts is

$$
v=\frac{\Lambda\left(u^{+}(t)\right)-\Lambda\left(u^{-}(t)\right)}{u^{+}(t)-u^{-}(t)} .
$$

If $u_{0} \in\left(L^{1}\left(\mathbb{R}^{N}\right) \cap L^{\infty}\left(\mathbb{R}^{N}\right)\right)^{+} \cap B V\left(\mathbb{R}^{N}\right)$, Lemma 6.1 gives sufficient conditions implying that $u \in B V_{\text {loc }}\left((0, T) \times \mathbb{R}^{N}\right)$ (see Proposition 6.2).

Under some additional assumptions we may derive from (6.10) a vertical contact angle condition. For that we assume that for $\mathcal{H}^{N-1}$ almost all $x \in J_{u(t)}$ there is a ball $B_{x}$ centered at $x$ such that either (a) $\left.u(t)\right|_{B_{x}} \geq \alpha>0$ or (b) $J_{u(t)} \cap B_{x}$ is the graph of a Lipschitz function with $B_{x} \backslash J_{u(t)}=B_{x}^{1} \cup B_{x}^{2}$ where $B_{x}^{1}, B_{x}^{2}$ are open and connected and $u(t) \geq \alpha>0$ in $B_{x}^{1}$ while the trace of $u(t)$ on $J_{u(t)} \cap \partial B_{x}^{2}$ computed from $B_{x}^{2}$ is zero.

In both cases, by Lemma 5.6 in [30], we have

$$
\left[\frac{\nabla \Phi(u)}{\sqrt{1+\beta|\nabla \Phi(u)|^{2}}} \cdot \nu^{J_{u(t)}}\right]_{+}=1 \quad \text { on } J_{u(t)} \cap B(x, r) .
$$

If (a) holds we also have

$$
\left[\frac{\nabla \Phi(u)}{\sqrt{1+\beta|\nabla \Phi(u)|^{2}}} \cdot \nu^{J_{u(t)}}\right]_{-}=1 \quad \text { on } J_{u(t)} \cap B(x, r) .
$$

Both conditions express in a weak way the vertical contact angle condition on the jump set of $u$.

For the proof, we adapt the proof of Proposition 8.1 in [30]. 
Proof: Let $w=\Phi(u(t)), \bar{\Lambda}=\Lambda^{\Phi}, \bar{T}=T \circ \Phi, \bar{S}=S \circ \Phi$. With this, we can write the entropy conditions as: for any $(S, T) \in \mathcal{T} \mathcal{S U B} \cup \mathcal{T S U P \mathcal { R }}$ and for $\mathcal{L}^{1}$-almost any $t>0$ the inequality

$$
\begin{aligned}
& {[S T \bar{\Lambda}(w(t))]_{+-}-\left[J_{T S \bar{\Lambda}^{\prime}}(w(t))\right]_{+-}} \\
& \quad \leq-v\left[J_{\bar{T} \bar{S}}(u(t))\right]_{+-}+\left[\left[\mathbf{z}(t) \cdot \nu^{J_{u(t)}}\right] T(w(t)) S(w(t))\right]_{+-}
\end{aligned}
$$

holds $\mathcal{H}^{N-1}$ a.e. on $J_{u(t)}$. Notice that, since $\Phi$ is continuous and strictly increasing, $J_{u(t)}=J_{w(t)}$.

Recall that, by Proposition 6.5, the Rankine-Hugoniot conditions are

$$
v[u]_{+-}=\left[\left[\mathbf{z} \cdot \nu^{J_{u(t)}}\right]\right]_{+-},
$$

where the flux $\mathbf{z}=\mathbf{a}(u, \nabla \Phi(u))$ is given by (6.1).

Let $\varepsilon>0$, be such that $w^{-}<w^{+}-\varepsilon<w^{+}$and let us choose $(S, T) \in \mathcal{T S U B}$ so that $S(r) T(r)=\left(r-\left(w^{+}-\varepsilon\right)\right)^{+}$. Then a simple computation shows that

$$
\begin{aligned}
& {[S T \bar{\Lambda}(w(t))]_{+-}=\bar{\Lambda}\left(w(t)^{+}\right) \varepsilon,} \\
& {\left[J_{T S \bar{\Lambda}^{\prime}}(w(t))\right]_{+-}=O\left(\varepsilon^{2}\right),} \\
& {\left[J_{\overline{T S}}(u(t))\right]_{+-}=O\left(\varepsilon^{2}\right),} \\
& {\left[\left[\mathbf{z}(t) \cdot \nu^{J_{u(t)}}\right] T(w(t)) S(w(t))\right]_{+-}=\varepsilon\left[\mathbf{z}(t) \cdot \nu^{J_{u(t)}}\right]_{+},}
\end{aligned}
$$

where $O\left(\varepsilon^{2}\right)$ is an expression such that $\left|O\left(\varepsilon^{2}\right)\right| \leq C \varepsilon^{2}$ for some $C>0$ and $\varepsilon>0$ small enough. Just comment that the second identity follows from

$$
\begin{gathered}
{\left[J_{T S \bar{\Lambda}^{\prime}}(w(t))\right]_{+-}=J_{T S \bar{\Lambda}^{\prime}}\left(w^{+}(t)\right)-J_{T S \bar{\Lambda}^{\prime}}\left(w^{-}(t)\right),} \\
J_{T S \bar{\Lambda}^{\prime}}\left(w^{-}(t)\right)=0, \text { and } \\
J_{T S \bar{\Lambda}^{\prime}}\left(w^{+}(t)\right)=\int_{w^{+}(t)-\varepsilon}^{w^{+}(t)}\left(r-\left(w^{+}(t)-\varepsilon\right)\right) \bar{\Lambda}^{\prime}(r) d r \\
\quad \leq \varepsilon\left(\bar{\Lambda}\left(w^{+}(t)\right)-\bar{\Lambda}\left(w^{+}(t)-\varepsilon\right)\right) \leq C \varepsilon^{2}
\end{gathered}
$$

for some $C>0$, since $\bar{\Lambda}$ is Lipschitz in $[a, b], 0<a<b$. For the third identity, observe that

$$
\begin{aligned}
{\left[J_{\overline{T S}}(u(t))\right]_{+-} } & =\int_{u^{-}(t)}^{u^{+}(t)}\left(\Phi(r)-\left(w^{+}(t)-\varepsilon\right)\right) d r \\
& =\int_{w^{+}(t)-\varepsilon}^{w^{+}(t)}\left(s-\left(w^{+}(t)-\varepsilon\right)\right)\left(\Phi^{-1}\right)^{\prime}(s) d s \\
& =\varepsilon\left(\left(\Phi^{-1}\right)^{\prime}\left(w^{+}(t)\right)-\left(\Phi^{-1}\right)^{\prime}\left(w^{+}(t)-\varepsilon\right)\right)=O\left(\varepsilon^{2}\right) .
\end{aligned}
$$


Then the entropy condition is written as

$$
\varepsilon\left(\bar{\Lambda}\left(w^{+}(t)\right)-\left[\mathbf{z} \cdot \nu^{J_{u(t)}}\right]_{+}\right) \leq O\left(\varepsilon^{2}\right) .
$$

Since $\left[\mathbf{z} \cdot \nu^{J_{u(t)}}\right]_{+} \leq \bar{\Lambda}\left(w^{+}(t)\right)$, this represents a contradiction unless

$$
\left[\mathbf{z} \cdot \nu^{J_{u(t)}}\right]_{+}=\bar{\Lambda}\left(w^{+}(t)\right)=\Lambda\left(u^{+}(t)\right) .
$$

In a similar way, we take $T=1$ and $S(r)=-\varepsilon$ if $r \leq w^{-}, S(r)=$ $r-\left(w^{-}+\varepsilon\right)$ if $r \in\left[w^{-}, w^{-}+\varepsilon\right], S(r)=0$ if $r \geq w^{-}+\varepsilon$. In this case, $(S, T) \in \mathcal{T} \mathcal{S U} \mathcal{P E R}$. Proceeding in the same way we have

$$
\begin{aligned}
& {[S T \bar{\Lambda}(w(t))]_{+-}=\bar{\Lambda}\left(w^{-}(t)\right) \varepsilon,} \\
& {\left[J_{T S \bar{\Lambda}^{\prime}}(w(t))\right]_{+-}=\varepsilon \omega_{\bar{\Lambda}}(\varepsilon),} \\
& {\left[J_{\overline{T S}}(u(t))\right]_{+-}=\varepsilon \omega_{\Phi^{-1}}(\varepsilon),} \\
& {\left[\left[\mathbf{z}(t) \cdot \nu^{J_{u(t)}}\right] T(w(t)) S(w(t))\right]_{+-}=\varepsilon\left[\mathbf{z} \cdot \nu^{J_{u(t)}}\right]_{-},}
\end{aligned}
$$

where $\omega_{\bar{\Lambda}}(\varepsilon), \omega_{\Phi^{-1}}(\varepsilon)$ are modulus of continuity of $\bar{\Lambda}$ and $\Phi^{-1}$, respectively. Thus the entropy condition is written as

$$
\varepsilon\left(\bar{\Lambda}\left(w^{-}(t)\right)-\left[\mathbf{z} \cdot \nu^{J_{u(t)}}\right]_{-}\right) \leq \varepsilon\left(\omega_{\bar{\Lambda}}(\varepsilon)+\omega_{\Phi^{-1}}(\varepsilon)\right) .
$$

This represents a contradiction unless

$$
\left[\mathbf{z} \cdot \nu^{J_{u(t)}}\right]_{-}=\bar{\Lambda}\left(w^{-}(t)\right)=\Lambda\left(u^{-}(t)\right) .
$$

Using the Rankine-Hugoniot condition given in Proposition 6.5, the speed of the front is

$$
v=\frac{\left[\mathbf{z} \cdot \nu^{J_{u(t)}}\right]_{+}-\left[\mathbf{z} \cdot \nu^{J_{u(t)}}\right]_{-}}{u^{+}-u^{-}}=\frac{\Lambda\left(u^{+}(t)\right)-\Lambda\left(u^{-}(t)\right)}{u^{+}-u^{-}} .
$$

We have proved (6.11).

Conversely, assume that (6.10) holds. Then

$$
\left[\left[\mathbf{z}(t) \cdot \nu^{J_{u(t)}}\right] T(w(t)) S(w(t))\right]_{+-}=[S T \bar{\Lambda}(w(t))]_{+-}
$$

and we may write the entropy conditions (6.12) as

$$
\frac{\Lambda\left(u^{+}(t)\right)-\Lambda\left(u^{-}(t)\right)}{u^{+}(t)-u^{-}(t)}\left[J_{\bar{T} \bar{S}}(u(t))\right]_{+-} \leq\left[J_{T S \bar{\Lambda}^{\prime}}(w(t))\right]_{+-} .
$$

Let us prove that $(6.15)$ hold for any $(T, S) \in \mathcal{T} \mathcal{S U B} \cup \mathcal{T S U P E R}$.

Let us observe that if $(T, S) \in \mathcal{T S \mathcal { B }}$, then $p=\bar{T} \bar{S}$ is a non-negative and non-decreasing function with $p(0)=0$. Then it suffices to prove that (6.15) holds for the functions $p(r)=\chi_{(d, \infty)}(r)$ since the closed convex cone generated by those functions contains all non-negative nondecreasing functions with $p(0)=0$.

Assume that $w^{-}>0$. If $d \leq w^{-}$, then $\left[J_{p}(u(t))\right]_{+-}=[u(t)]_{+-}$and $\left[J_{T S \bar{\Lambda}^{\prime}}(w(t))\right]_{+-}=[\bar{\Lambda}(u(t))]_{+-}$. Then $(6.15)$ holds. If $w^{-}<d \leq w^{+}$, 
then $\left[J_{p}(u(t))\right]_{+-}=u^{+}-\Phi^{-1}(d)$ and $\left[J_{T S \bar{\Lambda}^{\prime}}(w(t))\right]_{+-}=\Lambda\left(u^{+}(t)\right)-$ $\Lambda\left(\Phi^{-1}(d)\right)$. Then we may write $(6.15)$ as

$$
\frac{\Lambda\left(u^{+}(t)\right)-\Lambda\left(u^{-}(t)\right)}{u^{+}(t)-u^{-}(t)} \leq \frac{\Lambda\left(u^{+}(t)\right)-\Lambda\left(\Phi^{-1}(d)\right)}{u^{+}(t)-\Phi^{-1}(d)}
$$

and this holds because the convexity of $r \in[0, \infty) \rightarrow \Lambda(r)$. If $d>w^{+}$, then $\left[J_{p}(u(t))\right]_{+-}=0,\left[J_{T S \bar{\Lambda}^{\prime}}(w(t))\right]_{+-}=0$ and $(6.15)$ holds.

If $w^{-}=0$ then we may consider $d \geq 0$ and prove as above that (6.15) holds.

Let us observe that if $(T, S) \in \mathcal{T} \mathcal{S U P \mathcal { R }}$, then $p=\bar{T} \bar{S}$ is a nonpositive and non-decreasing function. Then it suffices to prove that (6.15) holds for the functions $p(r)=c+c^{\prime} \chi_{(d, \infty)}(r), c \leq 0,0 \leq c^{\prime} \leq|c|$, since the closed convex cone generated by those functions contains all non-positive non-decreasing functions.

Assume that $w^{-}>0$. If $d \leq w^{-}$, then $\left[J_{p}(u(t))\right]_{+-}=\left(c+c^{\prime}\right)[u(t)]_{+-}$ and $\left[J_{T S \bar{\Lambda}^{\prime}}(w(t))\right]_{+-}=\left(c+c^{\prime}\right)[\bar{\Lambda}(u(t))]_{+-}$. Then (6.15) holds. If $w^{-}<d \leq w^{+}$, then $\left[J_{p}(u(t))\right]_{+-}=c[u(t)]_{+-}+c^{\prime}\left(u^{+}(t)-\Phi^{-1}(d)\right)$ and $\left[J_{T S \bar{\Lambda}^{\prime}}(w(t))\right]_{+-}=c[\Lambda(u(t))]_{+-}+c^{\prime}\left(\Lambda\left(u^{+}(t)\right)-\Lambda\left(\Phi^{-1}(d)\right)\right)$. Then we may write $(6.15)$ as

$$
\begin{aligned}
& \frac{\Lambda\left(u^{+}(t)\right)-\Lambda\left(u^{-}(t)\right)}{u^{+}(t)-u^{-}(t)}\left(c[u(t)]_{+-}+c^{\prime}\left(u^{+}(t)-\Phi^{-1}(d)\right)\right) \\
& \leq c[\Lambda(u(t))]_{+-}+c^{\prime}\left(\Lambda\left(u^{+}(t)\right)-\Lambda\left(\Phi^{-1}(d)\right)\right),
\end{aligned}
$$

which, since $c^{\prime} \geq 0$, is implied by

$$
\frac{\Lambda\left(u^{+}(t)\right)-\Lambda\left(u^{-}(t)\right)}{u^{+}(t)-u^{-}(t)} \leq \frac{\Lambda\left(u^{+}(t)\right)-\Lambda\left(\Phi^{-1}(d)\right)}{u^{+}(t)-\Phi^{-1}(d)},
$$

which in turn holds because of the convexity of $r \in[0, \infty) \rightarrow \Lambda(r)$. If $d>$ $w^{+}$, then $\left[J_{p}(u(t))\right]_{+-}=c[u(t)]_{+-}$and $\left[J_{T S \Lambda^{\prime}}(u(t))\right]_{+-}=c[\Lambda(u(t))]_{+-}$ and (6.15) holds.

If $w^{-}=0$ then we may consider $d \geq 0$ and as above prove that (6.15) holds.

Remark 6.10. The previous results can be applied to the flux limited porous media equation

$$
u_{t}=\alpha \operatorname{div}\left(\frac{u^{r} \nabla u^{m}}{\sqrt{1+\beta\left|\nabla u^{m}\right|^{2}}}\right),
$$

where $m>0, r \geq 1, \alpha, \beta>0$. In this case, $\Phi(u)=u^{m}, \Lambda(u)=u^{r}$. Other qualitative properties including the existence of moving discontinuity 
fronts will be the object of a subsequent paper $[\mathbf{3 1}]$. We refer to $[\mathbf{4 8}]$ for a thorough study of the classical porous media equation.

Acknowledgements. The author acknowledges partial support by MICINN project, reference MTM2009-08171, by GRC reference 2009 SGR 773 and by "ICREA Acadèmia" prize for excellence in research funded both by the Generalitat de Catalunya.

\section{References}

[1] L. Ambrosio, N. Fusco, And D. Pallara, "Functions of bounded variation and free discontinuity problems", Oxford Mathematical Monographs, The Clarendon Press, Oxford University Press, New York, 2000.

[2] F. Andreu-Vaillo, V. Caselles, and J. M. Mazón, Existence and uniqueness of a solution for a parabolic quasilinear problem for linear growth functionals with $L^{1}$ data, Math. Ann. 322(1) (2002), 139-206. DOI: $10.1007 / \mathrm{s} 002080100270$.

[3] F. Andreu, V. Caselles, and J. M. Mazón, A strongly degenerate quasilinear equation: the elliptic case, Ann. Sc. Norm. Super. Pisa Cl. Sci. (5) 3(3) (2004), 555-587.

[4] F. Andreu, V. Caselles, and J. M. Mazón, A strongly degenerate quasilinear equation: the parabolic case, Arch. Ration. Mech. Anal. 176(3) (2005), 415-453. DOI: 10.1007/s00205-005-0358-5.

[5] F. Andreu, V. Caselles, and J. M. Mazón, A strongly degenerate quasilinear elliptic equation, Nonlinear Anal. 61(4) (2005), 637-669. DOI: 10.1016/j.na.2004.11.020.

[6] F. Andreu, V. Caselles, and J. M. Mazón, The Cauchy problem for a strongly degenerate quasilinear equation, J. Eur. Math. Soc. (JEMS) 7(3) (2005), 361-393. DOI: 10.4171/JEMS/32.

[7] F. Andreu, V. Caselles, and J. M. Mazón, Some regularity results on the 'relativistic' heat equation, J. Differential Equations 245(12) (2008), 3639-3663. DOI: 10.1007/10.1016/j.jde.2008.06. 024.

[8] F. Andreu, V. Caselles, J. M. Mazón, and S. Moll, Finite propagation speed for limited flux diffusion equations, Arch. Ration. Mech. Anal. 182(2) (2006), 269-297. DOI: 10.1007/s00205-0060428-3.

[9] F. Andreu, V. Caselles, J. M. Mazón, and S. Moll, The Dirichlet problem associated to the relativistic heat equation, Math. Ann. 347(1) (2010), 135-199. DOI : 10.1007/10.1007/s00208-0090428-3. 
[10] G. Anzellotti, Pairings between measures and bounded functions and compensated compactness, Ann. Mat. Pura Appl. (4) 135 (1983), 293-318 (1984). DOI: 10.1007/10.1007/BF01781073.

[11] Y. Atik And J. M. Rakotoson, Local T-sets and renormalized solutions of degenerate quasilinear elliptic equations with an $L^{1}$-datum, Adv. Differential Equations 1(6) (1996), 965-988.

[12] M. Bendahmane And K. H. Karlsen, Renormalized entropy solutions for quasi-linear anisotropic degenerate parabolic equations, SIAM J. Math. Anal. 36(2) (2004), 405-422. DOI: 10.1137/ S0036141003428937.

[13] Ph. BÉnilan, L. Boccardo, T. Gallouët, R. Gariepy, M. Pierre, And J. L. VÁzquez, An $L^{1}$-theory of existence and uniqueness of solutions of nonlinear elliptic equations, Ann. Scuola Norm. Sup. Pisa Cl. Sci. (4) 22(2) (1995), 241-273.

[14] Ph. BÉnilan And M. G. CRAndall, Regularizing effects of homogeneous evolution equations, in: "Contributions to analysis and geometry" (Baltimore, Md., 1980), Johns Hopkins Univ. Press, Baltimore, Md., 1981, pp. 23-39.

[15] Ph. BÉnilan and M. G. Crandall, Completely accretive operators, in: "Semigroup theory and evolution equations" (Delft, 1989), Lecture Notes in Pure and Appl. Math. 135, Dekker, New York, 1991, pp. 41-75.

[16] Ph. BÉnilan, M. G. Crandall, and A. Pazy, "Evolution Equations Governed by Accretive Operators", book in preparation.

[17] M. Bertsch And R. DAL PAsso, Hyperbolic phenomena in a strongly degenerate parabolic equation, Arch. Rational Mech. Anal. 117(4) (1992), 349-387. DOI: 10.1007/BF00376188.

[18] M. Bertsch AND R. DAl PAsso, A parabolic equation with a mean-curvature type operator, in: "Nonlinear diffusion equations and their equilibrium states, 3" (Gregynog, 1989), Progr. Nonlinear Differential Equations Appl. 7, Birkhäuser Boston, Boston, MA, 1992, pp. 89-97.

[19] $\mathrm{PH}$. Blanc, On the regularity of the solutions of some degenerate parabolic equations, Comm. Partial Differential Equations 18(5-6) (1993), 821-846. DOI: 10.1080/03605309308820952.

[20] Ph. Blanc, Sur une classe d'equations paraboliques degenerées a une dimension d'espace possedant des solutions discontinues, Ph.D. Thesis, Ecole Polytechnique Federale de Lausanne (1989).

[21] D. Blanchard And F. Murat, Renormalised solutions of nonlinear parabolic problems with $L^{1}$ data: existence and uniqueness, 
Proc. Roy. Soc. Edinburgh Sect. A 127(6) (1997), 1137-1152. DOI: 10.1017/S0308210500026986.

[22] D. Blanchard, F. Murat, and H. Redwane, Existence and uniqueness of a renormalized solution for a fairly general class of nonlinear parabolic problems, J. Differential Equations 177(2) (2001), 331-374. DOI: 10.1006/jdeq.2000. 4013.

[23] L. Boccardo, J. I. Díaz, D. Giachetti, and F. Murat, Existence of a solution for a weaker form of a nonlinear elliptic equation, in: "Recent advances in nonlinear elliptic and parabolic problems" (Nancy, 1988), Pitman Res. Notes Math. Ser. 208, Longman Sci. Tech., Harlow, 1989, pp. 229-246.

[24] L. Boccardo, D. Giachetti, J. I. Díaz, and F. Murat, Existence and regularity of renormalized solutions for some elliptic problems involving derivatives of nonlinear terms, J. Differential Equations 106(2) (1993), 215-237. DOI: 10.1006/jdeq.1993.1106.

[25] Y. Brenier, Extended Monge-Kantorovich theory, in: "Optimal transportation and applications" (Martina Franca, 2001), Lecture Notes in Math. 1813, Springer, Berlin, 2003, pp. 91-121. DOI: 10.1007/978-3-540-44857-0_4.

[26] F. E. Browder, Pseudo-monotone operators and nonlinear elliptic boundary value problems on unbounded domains, Proc. Nat. Acad. Sci. U.S.A. 74(7) (1977), 2659-2661.

[27] J. CARrillo, Entropy solutions for nonlinear degenerate problems, Arch. Ration. Mech. Anal. 147(4) (1999), 269-361. DOI: 10.1007/s002050050152.

[28] J. CARRILlo AND P. Wittbold, Uniqueness of renormalized solutions of degenerate elliptic-parabolic problems, J. Differential Equations 156(1) (1999), 93-121. DOI: 10.1006/jdeq.1998.3597.

[29] V. Caselles, An existence and uniqueness result for flux limited diffusion equations, Discrete Contin. Dyn. Syst. 31(4) (2011), 1151-1195. DOI: 10.3934/dcds.2011.31.1151.

[30] V. CAselles, On the entropy conditions for some flux limited diffusion equations, J. Differential Equations 250(8) (2011), 3311-3348. DOI : $10.1016 / j \cdot j$ de.2011.01.027.

[31] V. Caselles, On flux limited porous media diffusion equations, in preparation.

[32] G.-Q. Chen And H. Frid, Divergence-measure fields and hyperbolic conservation laws, Arch. Ration. Mech. Anal. 147(2) (1999), 89-118. DOI: $10.1007 / \mathrm{s} 002050050146$.

[33] A. Chertock, A. Kurganov, and P. Rosenau, Formation of discontinuities in flux-saturated degenerate parabolic equations, 
Nonlinearity 16(6) (2003), 1875-1898. DOI : 10.1088/0951-7715/ 16/6/301.

[34] M. G. CRAndall, Nonlinear semigroups and evolution governed by accretive operators, in: "Nonlinear functional analysis and its applications, Part 1" (Berkeley, Calif., 1983), Proc. Sympos. Pure Math. 45, Amer. Math. Soc., Providence, RI, 1986, pp. 305-337.

[35] M. G. Crandall and T. M. Liggett, Generation of semi-groups of nonlinear transformations on general Banach spaces, Amer. J. Math. 93 (1971), 265-298.

[36] G. DAL MAso, Integral representation on $\mathrm{BV}(\Omega)$ of $\Gamma$-limits of variational integrals, Manuscripta Math. 30(4) (1979/80), 387-416. DOI : $10.1007 / B F 01301259$.

[37] G. Dal Maso, F. Murat, L. Orsina, and A. Prignet, Renormalized solutions of elliptic equations with general measure data, Ann. Scuola Norm. Sup. Pisa Cl. Sci. (4) 28(4) (1999), 741-808.

[38] R. DAL PAsso, Uniqueness of the entropy solution of a strongly degenerate parabolic equation, Comm. Partial Differential Equations 18(1-2) (1993), 265-279. DOI : 10.1080/03605309308820930.

[39] V. De Cicco, N. Fusco, And A. Verde, On $L^{1}$-lower semicontinuity in BV, J. Convex Anal. 12(1) (2005), 173-185.

[40] E. De Giorgi And L. Ambrosio, New functionals in the calculus of variations, (Italian), Atti Accad. Naz. Lincei Rend. Cl. Sci. Fis. Mat. Natur. (8) 82(2) (1988), 199-210 (1989).

[41] J. J. Duderstadt And G. A. Moses, "Inertial confinement fusion", John Wiley \& Sons, New York, 1982.

[42] S. N. KružKov, First order quasilinear equations with several independent variables, (Russian), Mat. Sb. (N.S.) 81 (123) (1970), 228-255.

[43] R. J. MCCAnn And M. Puel, Constructing a relativistic heat flow by transport time steps, Ann. Inst. H. Poincaré Anal. Non Linéaire 26(6) (2009), 2539-2580. DOI: 10.1016/j.anihpc.2009.06.006.

[44] D. Mihalas and B. W. Mihalas, "Foundations of radiation hydrodynamics", Oxford University Press, New York, 1984.

[45] J.-M. Rakotoson, Uniqueness of renormalized solutions in a $T$ set for the $L^{1}$-data problem and the link between various formulations, Indiana Univ. Math. J. 43(2) (1994), 685-702. DOI: 10.1512/iumj.1994.43.43029.

[46] P. Rosenau, Free-energy functionals at the high-gradient limit, Phys. Rev. A 41(4) (1990), 2227-2230. DOI: 10.1103/PhysRevA. 41. 2227. 
[47] P. Rosenau, Tempered diffusion: A transport process with propagating fronts and inertial delay, Phys. Rev. A 46(12) (1992), R7371-R7374. DOI: 10.1103/PhysRevA.46.R7371.

[48] J. L. VÁzQuez, "The porous medium equation", Mathematical theory, Oxford Mathematical Monographs, The Clarendon Press, Oxford University Press, Oxford, 2007.

Departament de Tecnologies de la Informació i la Comunicació

Universitat Pompeu-Fabra

C/ Tànger, 122-140

08018 Barcelona

Spain

E-mail address: vicent.caselles@upf .edu

Primera versió rebuda el 12 de març de 2012, darrera versió rebuda el 29 d'octubre de 2012. 